\title{
Seismogenic effects in ULF/ELF/VLF electromagnetic waves
}

\author{
M. Hayakawa ${ }^{1,2}$, A. Schekotov ${ }^{3}$, J. Izutsu $^{4}$, and A. P. Nickolaenko ${ }^{5}$ \\ ${ }^{1}$ Hayakawa Institute of Seismo Electromagnetics Co. Ltd. (Hi-SEM), UEC Alliance Center \#521, 1-1-1Kojima-cho, \\ Chofu-shi, Tokyo, 182-0026, Japan \\ ${ }^{2}$ The University of Electro-Communications (UEC), Advanced Wireless \& Communications research Center \\ (AWCC), 1-5-1Chofugaoka, Chofu-shi, Tokyo, 182-8585, Japan \\ ${ }^{3}$ Institute of Physics of the Earth, Russian Academy of Sciences, 10 Bolshaya Gruzinskaya, Moscow, 123995, \\ Russia \\ ${ }^{4}$ Chubu University, International Digital Earth Applied Science Research Center, Kasugai, Aichi, Japan \\ ${ }^{5}$ Usikov Institute for Radio-Physics and Electronics, National Academy of Sciences of the Ukraine, 12 \\ Proskurast., Kharkov,61085, Ukraine
}

\begin{abstract}
There has been an enormous progress in the field of electromagnetic phenomena associated with earthquakes (EQs) and EQ prediction during the last three decades, and it is recently agreed that electromagnetic effects do appear prior to an EQ. A few phenomena are well recognizedas being statistically correlated with EQs: one is the lithospheric radio emission in the ULF (ultra-low frequency, $\mathrm{f}<1 \mathrm{~Hz}$ ) range, and the second is ionospheric perturbation not only in the lower ionosphere as seen by subionospheric VLF (very low frequency, $3 \mathrm{kHz}<\mathrm{f}<30 \mathrm{kHz}$ ) $/ \mathrm{LF}$ (low frequency, $30 \mathrm{kHz}<\mathrm{f}<300 \mathrm{kHz}$ ) propagation but also in the upper $\mathrm{F}$ region as detected by ionosondes, TEC observations, satellite observations, etc.

In addition to the above two phenomena, there have been several not-well-understood effects in possible association with EQs in the frequency ranges of ULF, ELF (extremely low frequency, $1 \mathrm{~Hz}<\mathrm{f}<3 \mathrm{kHz}$ ) and VLF:(i)ULF/ELF transients (or Q bursts), (ii) anomaly in Schumann resonance (SR), (iii) ULF magnetic field depression, (iv) EQ effect on Pc1 pulsations, (v) VLF electromagnetic emissions, (vi) EQ effect on VLF/ ELF whistlers, and (vii) Lightning. The purpose of this paper is to review observational results on those items by different workers. We initially present a case study and then a statistical study for each topic with paying particular attention to its correlation with EQs. All of those effects can be physically explained in terms of two effects: one is likely to be associated with ionospheric perturbation as additional signatures of the lithosphere-atmosphere-ionosphere coupling (LAIC) process ((ii), (iii), (iv) and (vi)) and the other is some kind of discharge phenomena in the atmosphere as by-products of the LAIC process ((i), (v), and (vii)).Then, we can describe possible explanations (or modeling) ever proposed for each item, even though those models are all at the stage of hypothesis and not well proven by
\end{abstract}


observations. Further studies of these phenomena help us better understand the LAIC mechanism of our current interest.

\section{Introduction}

This review deals with electromagnetic fields observed mainly on the ground in the ULF (ultralow frequency, $\mathrm{f}<3 \mathrm{~Hz}$ ), ELF (extremely low frequency, $3 \mathrm{~Hz}<\mathrm{f}<3 \mathrm{kHz}$ ) and VLF (very low frequency, 3 $\mathrm{kHz}<\mathrm{f}<30 \mathrm{kHz}$ ) bands. Depending on the field of research, different terminology is used to define the various frequency ranges, but we follow the frequency nomenclature employed in ionospheric and magnetospheric sciences. It is recently agreed that the observation of these ULF/ELF/VLF electromagnetic fields can provide us with important information not only on the upper atmosphere (ionosphere/magnetosphere), but also on the atmosphere and the Earth's crust(lithosphere) (e.g., Simoes et al., 2012; Pilipenko, 2012; Surkov and Hayakawa, 2014; Nickolaenko and Hayakawa, 2002, 2014; Hayakawa, 2015; Schekotov and Hayakawa, 2017).

We first discuss the main sources of these ULF/ELF/VLF noises. In the field of atmospheric electricity, the most famous one is the so-called Schumann resonances (SRs) (e.g., Sentman, 1995 ; Nickolaenko and Hayakawa, 2002, 2014; Price, 2016) covering the frequency range from 7-8 Hz to 30-35Hz, which are known to be electromagnetic resonance in the Earth-ionosphere cavity driven by the global lightning activity. The next source is ionospheric Alfven resonator (IAR) which is the resonance in the lower magnetosphere and whose eigen-frequency lies in the range from $0.5-0.25$ to $3-5 \mathrm{~Hz}$, but its generation mechanism is poorly understood, either related to lightning (atmospheric electricity topic) or to magnetospheric effect (space physics) (Pilipenko, 2012; Surkov and Hayakawa, 2014). The field-lineresonance and cavity mode eigen-frequencies of the magnetosphere are below this frequency range since they cover the interval of $10^{-2}-10^{-3} \mathrm{~Hz}$ which is even smaller than above resonance frequencies (Surkov and Hayakawa, 2014). These ULF waves are definitely of magnetospheric origin including Pc1 (continuous pulsation 1) due to the instability of ion Cyclotron waves in the magnetosphere (Saito, 1969; Jacobs, 1970; Nishida, 1972; Surkov and Hayakawa, 2014). In the ELF/VLF range, there is sferics (atmospherics) from lightning discharges. There are also some kinds of magnetospheric ELF/VLF emissions such as hiss and chorus, which are generated in the equatorial plane of the magnetosphere by plasma instabilities, and propagated along the magnetic field line towards an observer on the ground (Hayakawa and Sazhin, 1992; Sazhin and Hayakawa, 1992; Sazhin et al., 1993). Another popular 
ELF/VLF phenomenon is whistlers, which are originated from lightning discharges in the opposite hemisphere, propagate along the magnetic field line in the magnetosphere, penetrate through the ionosphere and observed on the ground in our hemisphere (Helliwell, 1965; Park, 1982; Hayakawa, 1995). Although the main contribution to ULF/ELF/VLF bands is from space physics and atmospheric electricity, it is found during the last three decades that electromagnetic phenomena with subtle intensity in these frequency ranges do take place mainly before the earthquakes (EQs) (see, e.g., Hayakawa (Ed), 1999; Hayakawa and Molchanov (Eds), 2002; Pulinets and Boyarchuk, 2004; Molchanov and Hayakawa, 2008; Hayakawa, 2015; Ouzounov et al. (Ed), 2018). In the field of this seismo-electromagnetics (electromagnetic phenomena associated with EQs) and EQ prediction, there are a few milestones. The first is the discovery that local high-amplitude ULF signals were observed near the epicenters of violent Ms (magnitude (surface))>7 EQs at Loma Prieta (Fraser-Smith et al., 1990) and Spitak (Kopytenko et al., 1990; Molchanov et al., 1992). Then, there was observed a clear signature of lower ionospheric perturbation for the 1995 Kobe EQ (M (magnitude) =7.3) with the use of VLF subionospheric propagation data (Hayakawa et al., 1996a; Molchanov and Hayakawa, 1998). The next milestone was the launch of a French satellite, DEMETER dedicated to seismo-electromagnetic studies in 2004, which has yielded a lot of scientific outputs on the lithosphere-atmosphere-ionosphere coupling (LAIC) (several summary papers can be found in Ouzounov et al. (Ed), (2018)). Considering these circumstances, the two topics of ULF lithospheric emissions and ionospheric perturbations (not only in the lower ionosphere but also in the F region) have been well studied and seem to be established at least observationally, even though the mechanisms of both are poorly understood. Please consult some reviews on ULF lithospheric emissions by Hayakawa et al., (2011a) and Hattori (2013), and some reviews or books on ionospheric perturbations are found in Pulinets and Boyarchuk (2004); Molchanov and Hayakawa (2008); Liu (2009); Sorokin et al., (2015); Hayakawa (2015); and Ouzounov et al. (Ed), (2018). So, these two main topics of seismo-electromagnetics are not addressed in this review, but other remaining topics in ULF/ELF/VLF are extensively studied here. In this review we will pay most attention to the following items of seismoelectromagnetic phenomena on the basis of ground-based measurements.

i. ULF/ELF transients (or Q bursts)

ii. Anomalies in SR

iii. ULF magnetic field depression 


\section{iv. EQ effect on Pc1 pulsations \\ v. VLF electromagnetic emissions \\ vi. EQ effect on ELF/VLF whistlers \\ vii. Lightning}

From the historical point of view the first four topics are relatively newly found, and so they seem to be even unpopular in the society of seismo-electromagnetics, while the latter three topics are not so new and attracted little attention recently, but worth describing here again. On the contrary, from the physical point of view, the topics of (ii), (iii), (iv) and (vi) might be another signature of LAIC process. The other topics, (i), (v) and (vii) are due to a kind of discharges as by-products of LAIC. We will review initially observational facts (case study and statistical study) for the above phenomenon one by one, and then we will present the possible explanation so far proposed for each item. Further understanding of these phenomena will contribute much to better understanding of the mechanism of LAIC of our current interest.

\section{ULF/ELF transients (or Q Bursts)}

In the field of atmospheric electricity, a Q-burst was first named in old days by Ogawa et al., (1967), which was known as an extremely intense impulsive ELF radiation, but its generation mechanism has been left unanswered for a long time as a mysterious event. In 1992 Williams et al., (1992) gave a new insight to this Q-burst, as it is closely associated with unconventional positive cloud-ground lightning discharges leading to the generation of transient luminous events (TLEs) in the mesosphere. In recent years Q bursts in association with lightning discharges and TLEs have been attracting a lot of attention in the fields of atmospheric electricity and space physics (e.g., Fullekrug et al. (Eds), 2006; Nickolaenko and Hayakawa, 2014).

In this section, we will present the similar kind of Q bursts in possible association with EQs, the socalled seismogenic Q bursts (impulsive ULF/ELF transients), which have been summarized in Schekotov et al., (2007, 2008, 2013) and Schekotov and Hayakawa (2017).The following descriptions are mainly based on the paper by Schekotov et al., (2013). 


\subsection{Observations at Kamchatka (Case study)}

\section{(i) Observation and analysis}

We use the ULF/ELF magnetic data observed at Kamchatka, so that we explain the equipment there. Variations of the ULF/ELF magnetic field are being measured at the Karymshino observatory, Kamchatka, Russia (Geographic lat $=52.83^{\circ} \mathrm{N}$, long=158.13 $\mathrm{E}$ ) since June 2000 with a 3-component induction magnetometer in the frequency band of $0.003-40 \mathrm{~Hz}$, with noise level $0.16^{*} \mathrm{f}^{-1} \mathrm{pT} / \sqrt{ } \mathrm{Hz}(\mathrm{f}$, frequency) and conversion function $0.4 * \mathrm{fV}^{*} \mathrm{~Hz} / \mathrm{nT}$ in the frequency band $\mathrm{f}=0.003-4 \mathrm{~Hz}$ and $1.6 \mathrm{~V} / \mathrm{nT}$ in the band $\mathrm{f}=4-40 \mathrm{~Hz}$. The sensors for measuring the horizontal components $\mathrm{H}$ and $\mathrm{D}$ are oriented along the magnetic meridian and transversally to it, and the $\mathrm{Z}$ means vertical. The parameters of all three sensors are identical with accuracy less than $3 \%$ in the conversion function module and $2^{\circ}$ in the phase characteristics. These discrepancies are corrected with the help of calibration circuits.

The output signal is digitized with a sampling frequency of $150 \mathrm{~Hz}$ with the 24-bit data acquisition system (DAS) and written on DAS hard disk. The data are copied and sent to the observatory for further analyses.

We first perform the preliminary routine data processing, which includes the correction of non-physical data and data gaps, filtration and decimation with the sampling frequency of $50 \mathrm{~Hz}$. Then, to estimate the spectral and polarization characteristics of signals, we calculate their power spectral densities and crossspectral densities: $P_{h h}, P_{d d}, P_{z z}, P_{h d}$ and $P_{d h}$.In evaluating the average characteristics of signals we use their Fourier transforms with frequency resolution of $\sim 0.2 \mathrm{~Hz}$ and time window of $30 \mathrm{~min}$, but for impulsive transient signals we use a wave let transform based on a 5-order complexGaussian wavelet.

We compute the parameters of polarization ellipse using the conventional procedure (Fowler et al., 1967) and define the angle $\theta$ that the principal axis of polarization ellipse makes with the $\mathrm{H}$-axis, or the orientation of polarization ellipse according to the following formula:

$$
\tan 2 \theta=\frac{2 \operatorname{Re} P_{h d}}{P_{h h}-P_{d d}}
$$

Re in the numerator means real part. Taking into account the signs of the numerator and denominator, the angle $\theta$ is defined in the interval $[-\pi / 2, \pi / 2]$ in the usual manner. We can also compute the parameters describing the ellipticity and sense of polarization ellipse in terms of the angle $\beta$. 


$$
\beta=-\frac{1}{2} \arcsin \frac{2 \operatorname{Im} P_{h d}}{\sqrt{\left(P_{h h}-P_{d d}\right)^{2}+4\left|P_{h d}\right|^{2}}}
$$

Here, Im means imaginary part. The ellipticity or the ratio of minor to major axis is defined by $\tan \beta$ and the sense of polarization by the sign of $\beta$, i.e. $\beta>0$ or $\beta<0$ as the polarization being right-hand (R) or left-handed (L) as measured when looking into the propagation wave. The ellipticity varies from -1 (L) to $+1(\mathrm{R})$ with zero for the linear polarization.

Seismic data are taken from the seismic catalogue and the local seismic index $K_{S}$ (Molchanov et al., 2003; Molchanov and Hayakawa, 2008) for each EQ is calculated. It is proportional to the EQ seismic energy in the observational point and computed by the following equation,

$$
K_{s}=10^{0.75 M s} /(R+100)
$$

where $\mathrm{R}$ is the distance in $\mathrm{km}$ from the observational point. And the geomagnetic activity is estimated with the conventional daily $\Sigma K_{P}$ index.

In the frequency range of ULF the amplitude of geomagnetic fluctuations decreases rapidly with frequency in such a way of 3 orders lower at $1 \mathrm{~Hz}$ than at $0.01 \mathrm{~Hz}$. At higher frequencies the level of natural geomagnetic emissions is much lower, but artificial interferences dominate at frequencies above $50 \mathrm{~Hz}$. Thus it is reasonable to choose the frequency range from a few $\mathrm{Hz}$ to a few tens of $\mathrm{Hz}$ to look for weak signals associated with seismicity. Here are the data analyzed of more than 3-year monitoring of the magnetic field fluctuations in the frequency range $1-40 \mathrm{~Hz}$ in a seismically active region at Kamchatka. We compare different parameters of natural ULF/ELF emissions for the seismically active and quiet time intervals and select the field parameters, which are more sensitive to seismicity.

\section{(ii) Analysis result}

To select the best among the ULF/ELF parameters, the record was studied in the interval of about 1.5 months long around the seismic swarm in the middle of March, 2003 as a case study. We choose this interval to study a relation between the seismicity and ULF/ELF field variations because of their remarkable behavior. Because the first half of the interval is seismically absolutely quiet, and the second half starts with the Ms $=5.9$ shock on March, 15. This EQ is the first in the EQ series with slowly decreasing intensity. The second peak in seismic activity corresponds to Ms $=6$ EQ registered on March, 
19. Epicenters of almost all the EQs lie in the sea, east of the observation point. Seismic and geomagnetic activity and ULF/ELF field parameters are summarized in Figure1.

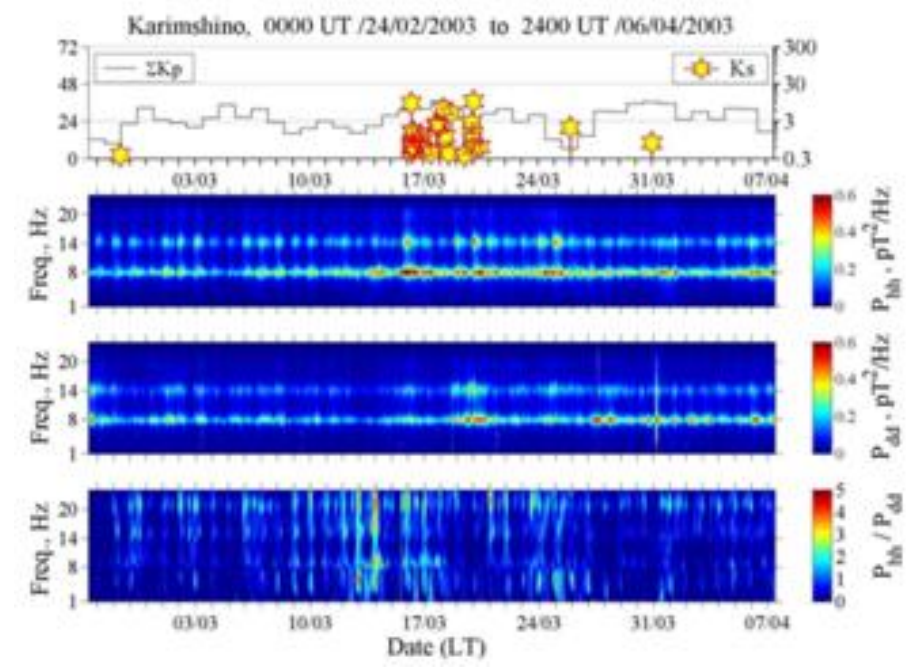

Figure1: Upper panel indicates $K_{S}$ (seismic index) and $\Sigma K_{p}$ (index of global geomagnetic activity). The second and third panels refer to the spectra of horizontal components of the magnetic field, $\mathrm{H}$ and $\mathrm{D}$, and the bottom panel, the spectral ratio $P_{h h} / P_{d d}$. Reproduced from Schekotov et al., (2013) with permission from TERRAPUB.

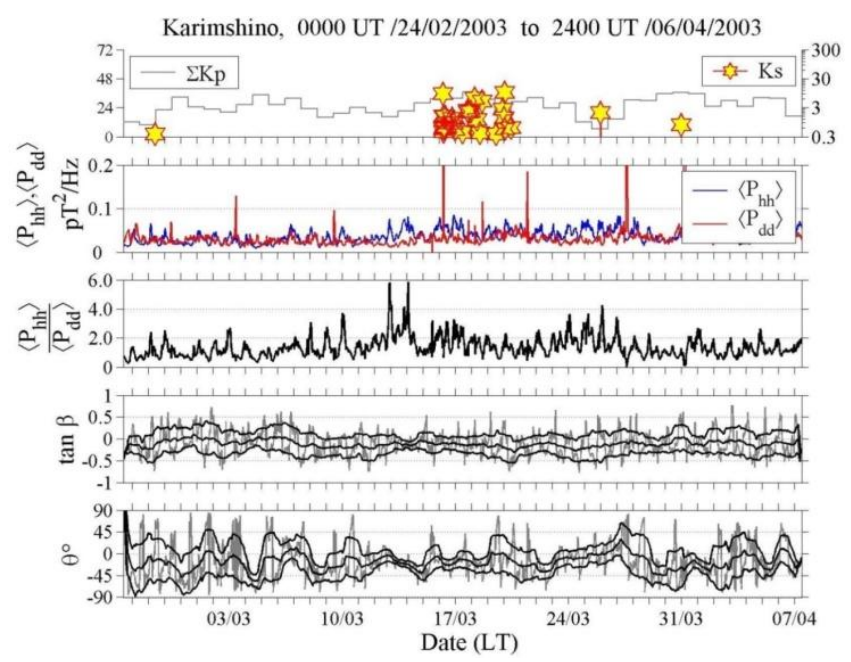

Figure 2: Characteristics of ULF/ELF noise in the frequency band $4-6 \mathrm{~Hz}$ for the same period of observation. From top to bottom: seismic index $(\mathrm{Ks})$, index of the global geomagnetic activity $\left(\Sigma K_{p}\right)$ and power spectral densities, spectral ratio, the signal ellipticity, and the orientation of polarization ellipse. In the bottom two panels gray lines show the current value of parameters $(\tan \beta, \theta)$, medium black line is a running mean value ( 24 hours window), and upper and lower black lines show the $\pm 1 \sigma$ (standard deviation) deviation from the mean value. Reproduced from Schekotov et al., (2013) with permission from TERRAPUB. 
It is seen from Figure1 that we have an interval with the enhanced $P_{h h} / P_{d d}$ ratio starting several days prior to an EQ and lasting several days afterwards. A similar but weaker effect is noticed in the power spectra of the field components. Such behavior of spectral parameters may correspond to a source located eastward (westward) from the observation point. Coincidence of the intervals with high seismicity and steep variations of the field parameters makes the assumption plausible on a physical relation between them. Namely, we can assume that an additional local source of ULF/ELF magnetic field fluctuations appears in the epicenter zone during the last stage of the EQ preparation and after the EQ. Characteristics of the ULF/ELF geomagnetic field in the frequency bands aside of SRs are shown in Figures1 and 2. The indices of seismic $K_{S}$ and daily global geomagnetic activity $\Sigma K_{p}$ are given in the upper panel. Then, from top to bottom we plot the power spectral density of the horizontal components of the magnetic field $\left(P_{h h}, P_{d d}\right)$, their spectral ratio $\left(P_{h h} / P_{d d}\right)$, ellipticity and ellipse orientation. Figure 2 illustrates the results for the frequency band 4-6 Hz below the fundamental harmonic of SR, while Figure 3 gives the same parameters for the frequency band $20-24 \mathrm{~Hz}$ above the third harmonic of SR.

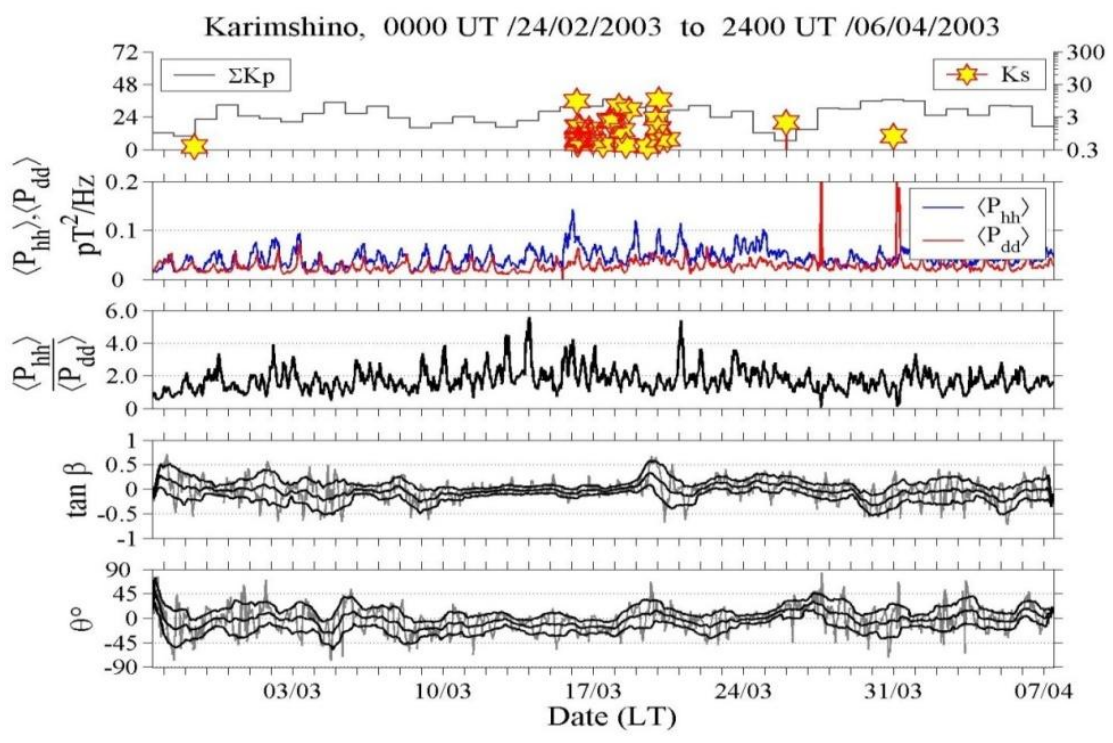

Figure 3: The same as Figurer 2, but for the frequency range of $=20-24 \mathrm{~Hz}$. Reproduced from Schekotov et al., (2013) with permission from TERRAPUB. 
In both frequency bands the spectral power of H-component and the ratio increase 3-4 days before the first shock of the EQ-swarm started on March, 15, 2003. Simultaneously, we find decreases in the absolute value of ellipticity and the standard deviation $(\sigma)$ of both ellipticity and ellipse orientation angle. For the further analysis, we take the nighttime intervals of \pm 5 hours around the local midnight and calculate the mean values of the power spectral densities, their ratio and standard deviations (rms) of the ellipticity and ellipse orientation angle in the frequency band $4-6 \mathrm{~Hz}$. Different combinations of spectral and polarization parameters were tested to select a parameter which is most sensitive to seismicity. The behavior of different combinations of spectral and polarization parameters is summarized in Figure 4.

Karimshino, 0000 UT /24/02/2003 to 2400 UT /06/04/2003

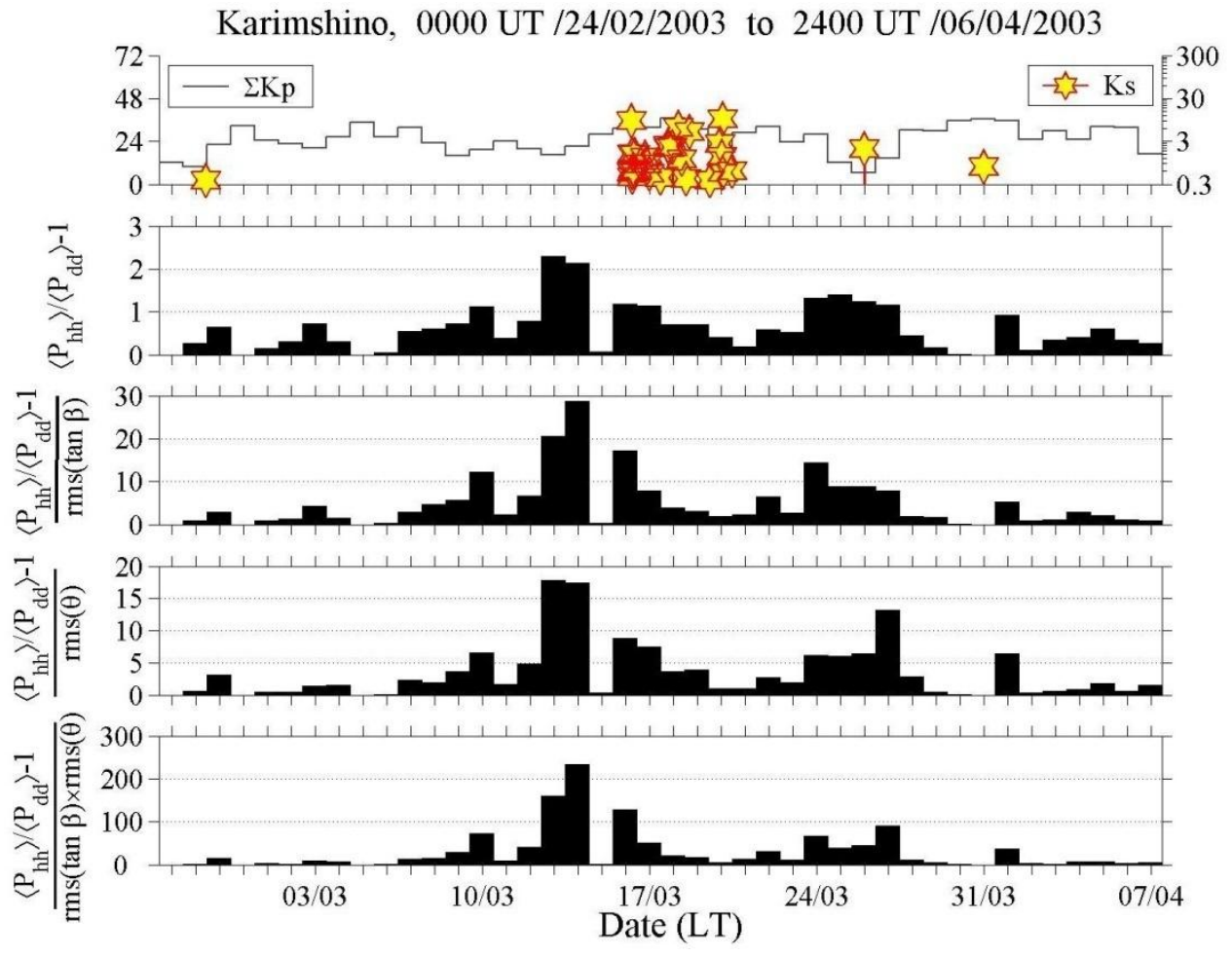

Figure 4: Seismicity, geomagnetic activity and parameters of the magnetic field over nighttime intervals in the frequency band 4-6 Hz. The upper panel indicates the $K_{S}$ seismic index, $\Sigma K_{p}$ (index of the global geomagnetic activity).Panels 2 to 5 refer to $P_{h h} / P_{d d}-1, \Delta S=\left(P_{h h} / P_{d d}-1\right) / r m s(\tan (\beta)), \Delta S_{t}=\left(P_{h h} / P_{d d}-1\right) / r m s(\theta)$, $\Delta S_{\beta t}=\left(P_{h h} / P_{d d}-1\right) /(r m s(\tan (\beta)) \times r m s(\theta))$.Reproduced from Schekotov et al., (2013) with permission from TERRAPUB.

It is found in Figure 4 that sensitivities of all the parameters exceed that of $\left(P_{h h} / P_{d d}-1\right)$ and are approximately equivalent. However, the lateral extension of a source and its explicit position influence 
the ellipse orientation rather than the ellipticity. Taking into account that the location and the size of a source can vary within a limited zone, we have chosen the following parameter $\Delta S$, which presents the seismic influence better than any other parameters.

$$
\Delta S=\frac{\left(\frac{P_{h h}}{P_{d d}}-1\right)}{r m s(\tan (\beta))}
$$

The results of comparison of the efficiency of two parameters $\Delta S$ and $P_{h h} / P_{d d}-1$ are shown in Figure 5(a) for the interval of 2003.02.24 - 2003.04.06 and in Figure 5(b) for the interval of 2004.07.12 2004.08.08. Both parameters are found to increase with seismicity, but the time correspondence of $\Delta S$ enhancements with the groups of EQs is really amazing. The parameter $P_{h h} / P_{d d}-1$ demonstrates several peaks in the seismically quiet intervals, which correspond to low amplitudes and/or unstable polarization of the signal. The relevant peaks are suppressed when using the parameter $\Delta S$. Thus, the enhanced $\Delta S$ indicates the appearance of an additional signal with the polarization ellipse oriented along the magnetic meridian and the ellipticity stable at time scales of several hours. In a more general case of arbitrary direction to the source $P_{h h}$ and $P_{d d}$ should be changed by $P_{n n}$ and $P_{t t}$, respectively, where indices n and $\mathrm{t}$ correspond to the directions perpendicular and parallel to the direction from the observation point to the source, respectively. The effect is recognized in the clearest way in the frequency band of $4-6 \mathrm{~Hz}$.
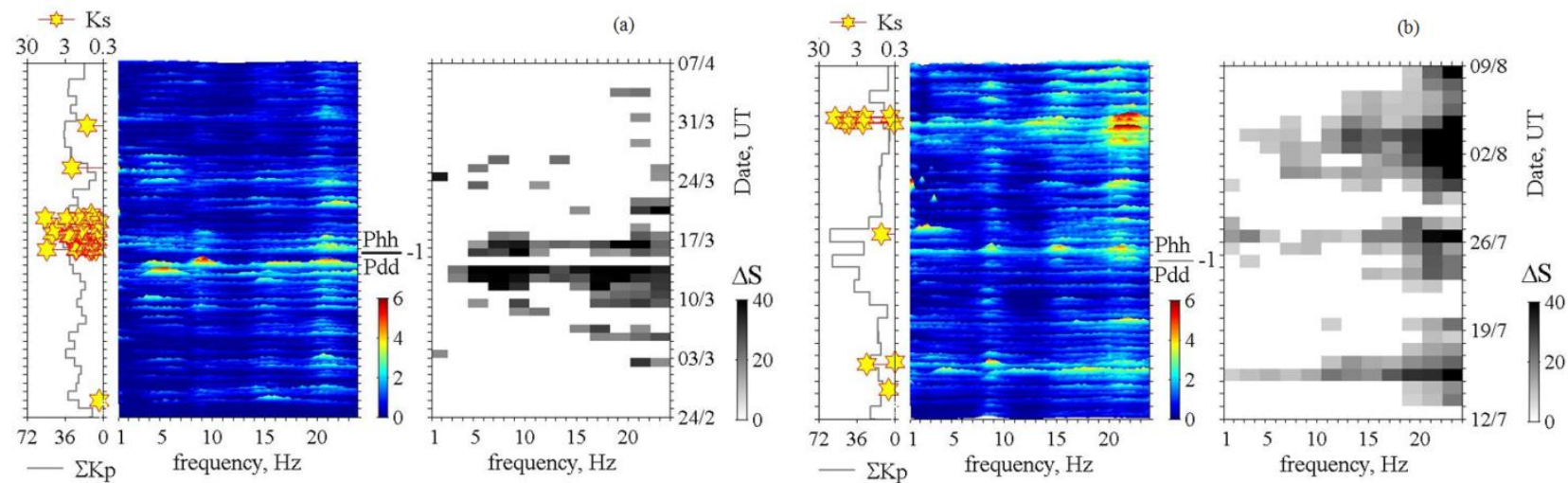

Figure 5(a): Left: Seismicity and geomagnetic activity, middle: $\left(\mathrm{P}_{\mathrm{hh}} / \mathrm{P}_{\mathrm{dd}}-1\right)$, and right: $\Delta \mathrm{S}$ averaged over nighttime intervals in the frequency band $1-24 \mathrm{~Hz}$ for the temporal interval of February, 24 to April, 6, 2003 (time goes from bottom to top). (b) The same as (a), but for the interval of July, 12 to August, 8, 2004.Reproduced from Schekotov et al., (2013) with permission from TERRAPUB. 


\subsection{Statistical study at Kamchatka and a trial of EQ forecast with $U L F / E L F$ radiation}

As a statistical study, the spectral density of the total horizontal power $G=\left\langle P_{h h}\right\rangle+\left\langle P_{d d}\right\rangle$ for the whole period of observation is plotted in Figure 6. Two indices of local seismic $K_{S}$ and global geomagnetic $\Sigma K_{p}$ activity are again shown in the upper panel, and $\Delta S$ and $G$ are given in the middle and bottom panels, respectively. They are calculated with 2-day averaging over night hours in the frequency band of 4-6 Hz. The total horizontal spectral power indicates a typical seasonal variation with maximum at local summer. On the other hand, the seasonal variation is not obvious in the variation of $\Delta S$, which suggests an evident correlation with seismic activity. Five intervals of high seismicity are clearly seen in the upper panel and each of them corresponds to the interval when the parameter $\Delta S$ exhibited a significant enhancement. It is also important that the parameter $\Delta S$ is not influenced by geomagnetic activity.

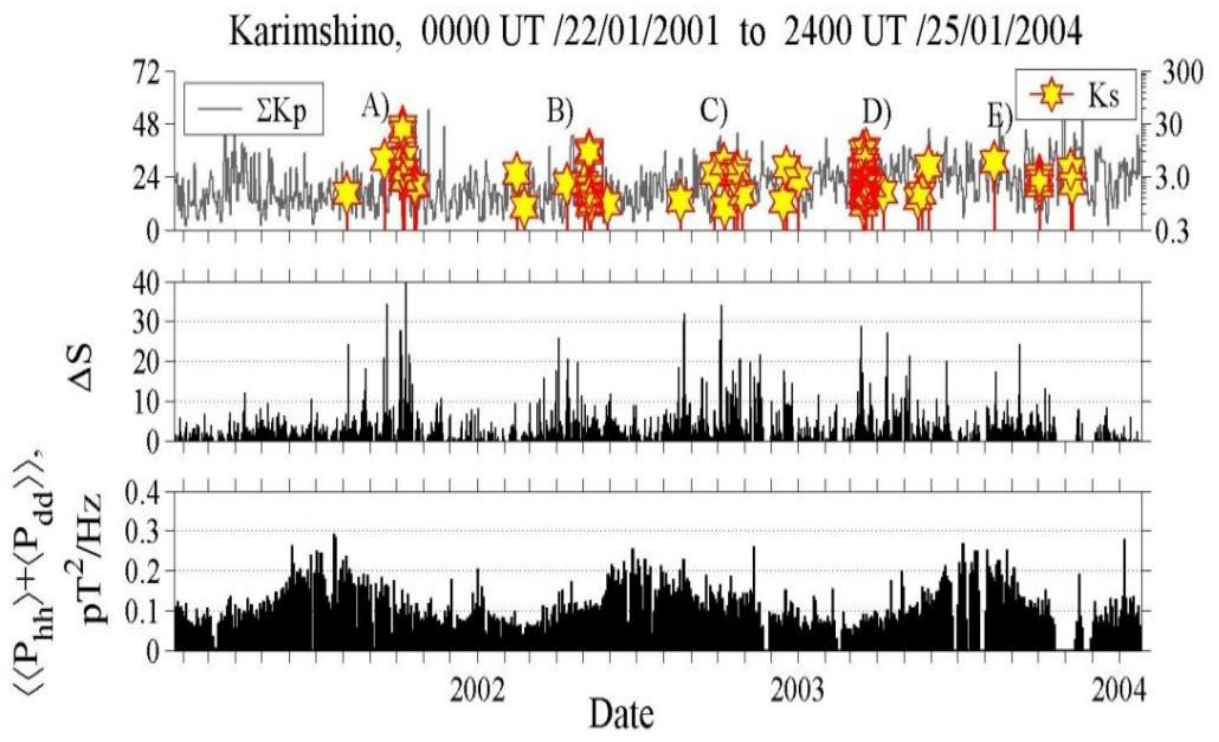

Figure 6: (Upper) Seismic and geomagnetic activity, (middle) $\Delta$ Sand (bottom) the total horizontal spectral power for the whole period of observations. Field parameters are calculated in the frequency range of $4-6 \mathrm{~Hz}$ for local nighttime. Reproduced from Schekotov et al., (2013) with permission from TERRAPUB.

The influence of individual EQs on $\Delta S$ is illustrated in Figure 6. It is seen from the figure that the EQs located to the east of the observatory at distances $\mathrm{R}<300 \mathrm{~km}$ contribute mostly to the variation in $\Delta S$. Due to the specific distribution of EQs in the observation region, these eastward EQs are simultaneously closest to Karymshino as shown in Figure 7. 


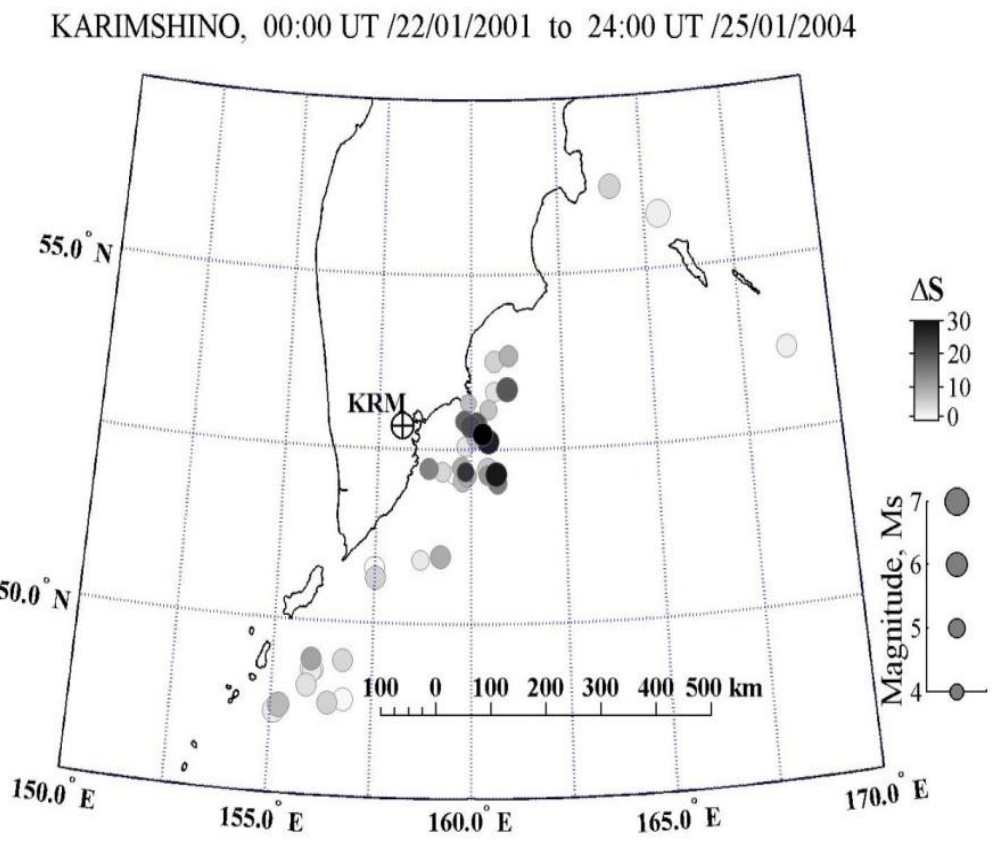

Figure7: Map of the main shocks in the vicinity of Karymshino (shown with a crossed circle and station code KRM). EQ epicenters are shown with circles. The color (in divisions of gray) corresponds to the maximal during the last 5 days before an EQ, and the size is proportional to the EQ magnitude. Only the EQs with $>1$ and depths $\mathrm{H}$ $<50 \mathrm{~km}$ are shown. Reproduced from Schekotov et al., (2013) with permission from TERRAPUB.

The analysis of data for individual EQs shows that the following EQ parameters correspond to noticeable changes in the magnetic field polarization and especially in $\Delta S:$ depths $H<50 \mathrm{~km}$, magnitudes $M s>5.5\left(\mathrm{E}>10^{13} \mathrm{~J}\right)$ and epicentral distances $\mathrm{R}<300 \mathrm{~km}$. Finally, let us estimate the reliability of the effect by using a conventional approach, which was developed to estimate the seismic precursor efficiency. We reproduce the definitions by Console (2001):

- Target volume $V_{t}$ is a volume in 3-D (three dimensional) space (time and 2 coordinates of the Earth's surface) determined by the time of observation and geographical area of observation. Each EQ with pre-conditioned magnitude threshold or target event is depicted as a point in the volume $V_{t}$.

- Alarm volume $V_{A}$ is a volume in which an EQ related to that precursor is expected.

- If an EQ occurs in the alarm volume, it is called a success (S).

- If an EQ occurs outside the alarm volume, it is a failure of prediction.

- An alarm that is not associated to any target EQ is called a false alarm. 
If $N_{S}, N_{A}$ and $N_{E}$ are the number of success, the number of alarms and the total number of EQs in the target volume, then parameters commonly considered in EQ prediction evaluation are as follows:

- Success rate $=N_{S} / N_{A}$ is the rate at which precursors are followed by target events in the alarm volume.

- False alarm rate $=1-N_{S} / N_{A}$ is the rate at which precursors are not followed by target events.

- Alarm rate $=N_{S} / N_{E}$ is the rate at which target events are preceded by precursors.

- Failure rate $=1-N_{S} / N_{E}$

- Probability gain $P G=\left[N s /\left(N_{A} V_{A}\right)\right] /\left[N_{E} / V_{t}\right]$ is the ratio between the rate at which target events occur in the alarm volume and the average rate at which target events occur over the whole target volume.

Generally, a precursor can be considered as reliable if it achieves a PG value greater than unity (Console, 2001). In our case of single station observation both the target volume and the alarm volume turn into the time intervals, hence PG relation can be rewritten as follows:

$$
P G=\left[T e /\left(T_{A} N_{A}\right)\right]\left(N_{S} / N_{E}\right)
$$

Hère $T e$ is the observation time and $T_{A}$ is the alarm interval.

To estimate the timescale of the $\Delta S$ pre-seismic variation and its threshold level $\Delta S_{t h}$ that maximizes the PG, we use a conventional superposed epoch (SPE) analysis with the dates of main shocks with $K_{S}>1$ taken as centers of time intervals. The analysis of the PG in dependence on the $\Delta S_{t h}$ shows that there occur noticeable changes in the interval \pm 5 days around the EQ date and the optimal threshold value of $\Delta S$ is $\Delta S_{t h} \approx 10$ for chosen parameters of analysis. The results of SPE analysis for 16 intervals with $K_{S}>1$ major EQs and max $(\Delta S(\tau))>\Delta S_{t h}$ (where $-15<\tau<0$ and $\tau$ is time in days from the EQ day) are shown in Figure 8. 


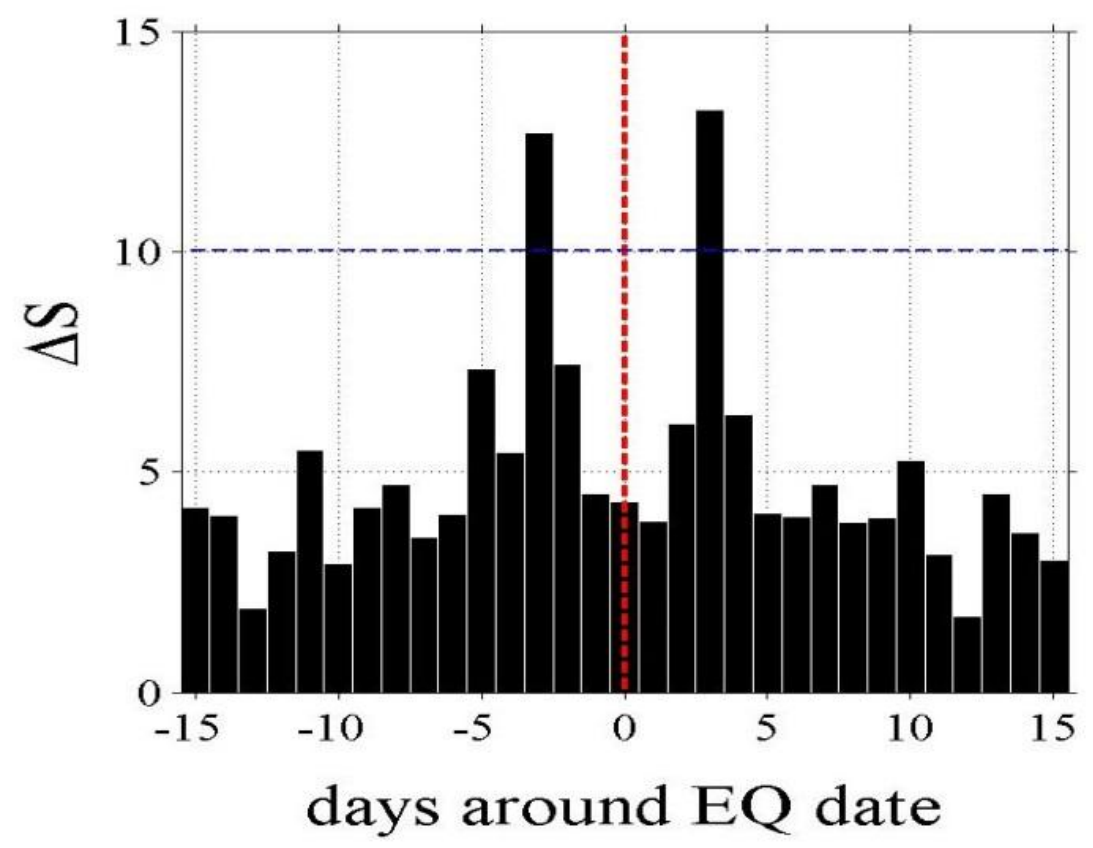

Figure 8: $\Delta S$ variation \pm 15 days in the vicinity of 16 main shocks with $K_{S}>1$ and $\max (\Delta S(\tau))>\Delta S_{t h}$. Reproduced from Schekotov et al. (2013), with permission from TERRAPUB.

Figure 8 shows that the effect is almost symmetrical about the moment of the first shock and the leading time is about 5 days. We calculate the daily averaged $\Delta S$ and use the following rules:

- An interval with $\Delta S$ exceeding the threshold level $\Delta S_{t h} \approx 10$ is considered an alarm interval.

- The duration of the alarm interval is 5 days after the start day.

- The anomalies occurred from the main shock until 5 days after it are considered as being associated with the aftershock activity and are excluded from our consideration.

The application of these simple rules to the real observational data (e.g., the year of 2001) is shown in Figure 9, in which black circles on the abscissa indicate EQs without any precursor, filled red squares correspond to success and empty squares, false alarms. The results of the analysis of the effectiveness of the method for EQ prediction are summarized in Table 1 for each year. 


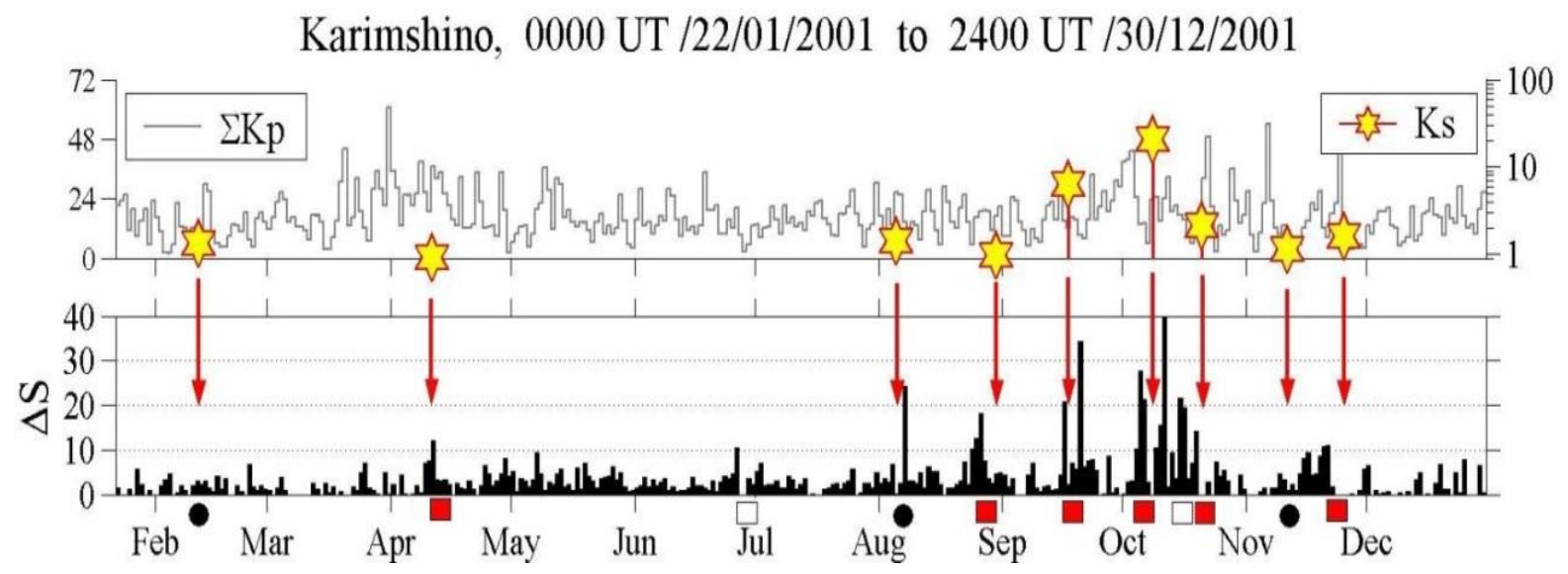

Figure 9: A trial of the EQ prediction technique for an interval from January 22, 2001 to December 30, 2001. Upper panel: EQs with $K_{S}>1$ (hexagonal stars), $\Sigma K_{p}$ daily indices, lower panel: $\Delta S$. Alarm intervals and EQs are shown by markers above the horizontal axis. Red squares correspond to success, and empty squares correspond to false alarms, and black circles indicate EQs without precursors. Reproduced from Schekotov et al., (2013), with permission from TERRAPUB.

Table 1 Summary of $\Delta S$ efficiency as an EQ precursor for 3 years

\begin{tabular}{|l|c|c|c|c|c|c|c|}
\hline $\mathrm{N}$ & $\begin{array}{c}\text { Observation } \\
\text { Period } \\
\text { Te, days }\end{array}$ & $\mathrm{N}_{\mathrm{E}}$ & $\mathrm{N}_{\mathrm{A}}$ & $\mathrm{N}_{\mathrm{S}}$ & $\begin{array}{c}\text { Success } \\
\text { Rate }\end{array}$ & $\begin{array}{c}\text { Alarm } \\
\text { Rate }\end{array}$ & $\begin{array}{c}\text { Probability } \\
\text { Gain }\end{array}$ \\
\hline $1(2001)$ & 343 & 9 & 7 & 6 & 0.85 & 0.66 & 6.47 \\
\hline $2(2002)$ & 364 & 15 & 14 & 7 & 0.50 & 0.47 & 2.45 \\
\hline $3(2003)$ & 264 & 10 & 13 & 3 & 0.23 & 0.30 & 1.22 \\
\hline Total & 971 & 34 & 34 & 16 & 0.47 & 0.47 & 2.68 \\
\hline
\end{tabular}

The overall PG value for each interval of observation is presented in the last column of Table 1. All of them arefound to be greater than unity,which suggests the usefulness of this method as a short-term EQ precursor.

Not only the time but also the place of an EQ can be determined from the analysis of the characteristics of this radiation (Schekotov et al.,2008). As anexample, Figure10 illustrates the result of data processing in anticipation of the start of the swarm on March 15. Here, in addition to determining the time of this 
start, we attempt to determine its azimuth. The three rectangular panels from top to bottom are: $\mathrm{K}_{\mathrm{S}}$ seismic index, spectrum of $\Delta S(f)$ and its averaged value $\langle\Delta S(f)\rangle$. With the round panels we show maps in azimuthal equidistant projection centered at the observation point. Here we also plot the histogram of azimuths of the radiation.In the bottom three circular panels are maps in azimuthal equidistant projection centered at the observatory for the 3,2 and1day before the swarm and on the right top circular panel on the day it started.We find that on the day of March 13 when $\langle\Delta S(f)\rangle$ reaches a maximum peak of the azimuthal distribution, it coincides with the azimuth of the EQ happened on March 15.

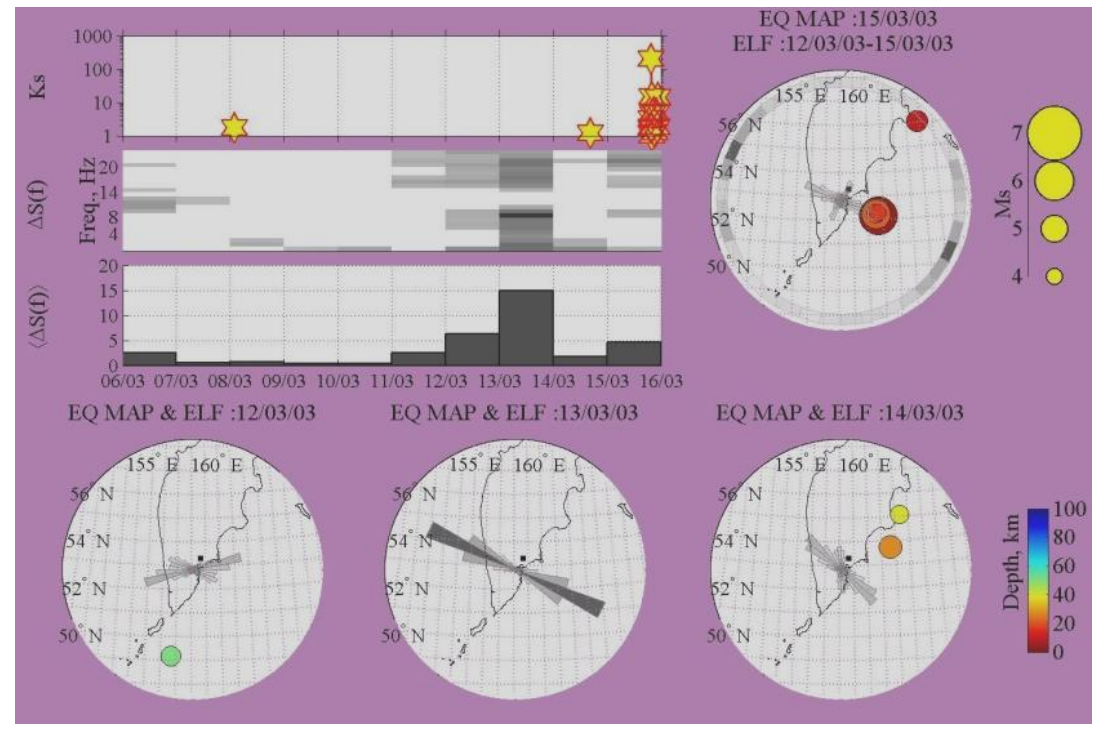

Figure10 : Three rectangular panels from top to bottom are $K_{S}$ seismic index, spectrum of $\Delta S(f)$ and its averaged value $\langle\Delta S(f)\rangle$.With the round panels we show maps in azimuthal equidistant projection centered at the observation point. The histogram of azimuths of detected radiation is also shown. The three circle bottom panels illustrate the histogram of azimuthal distributions for 3,2, and 1 day before the first EQ and in the top right panel on the day of the EQ. After Schekotov et al. (2013) with permission from TERRAPUB.

Let us summarize basic properties of the observational results:

- A few days before an EQ there appear the impulsive emissions in a frequency range from a few to a few tens of Hertz,

- The combined characteristic of the field $\Delta S=\left(P_{h h} / P_{d d}-1\right) / r m s(\tan (\beta))$ proved to be most sensitive to this radiation, 
- Lead time in $\Delta S$ depends on the frequency and it varies from1-5 days in units of Hertz up to 1-2 weeks for tens of Hertz,

- On average, it reaches a maximum three days before the EQ,

- Azimuth of the radiation source calculated for impulsive component of the magnetic field is approximately equal to the directionto the epicenter of future EQs,

- A statistical relation with EQs demonstrates the usefulness of the method as a short-term EQ precursor.

\subsection{A further example for the 2011 Tohoku $E Q$}

We have presented a statistical study on the correlation between linearly polarized Q bursts with EQs on the basis of Kamchatka observations, and here we will present our latest result of those Q bursts for a particular recent and disastrous 2011 Tohoku EQ, which was based on the paper by Ohta et al., (2013).

\section{(i) Observation system and observation network}

The geomagnetic data used here have been obtained by the Chubu University ULF/ELF network which consists of three observatories; Shinojima (abbreviated as SHI; geographic coordinates, $34.67^{\circ} \mathrm{N}$, $\left.137.01^{\circ} \mathrm{E}\right)$, Nakatsugawa (NAK, $\left.35.42^{\circ} \mathrm{N}, 137.55^{\circ} \mathrm{E}\right)$ and Izu (IZU, $\left.34.64^{\circ} \mathrm{N}, 138.85^{\circ} \mathrm{E}\right)($ Hata et al., 2010). Figure 11 illustrates the relative locations of three ULF/ELF observatories and the epicenter of the 2011 Japan EQ (11/03/11, the biggest orange circle). Also, we have plotted, in Figure 11, one representative observatory of Kakioka (KAK) belonging to Japan Meteorological Agency (JMA), and some other foreshocks (09/03/11) and aftershocks (11/03/11). 


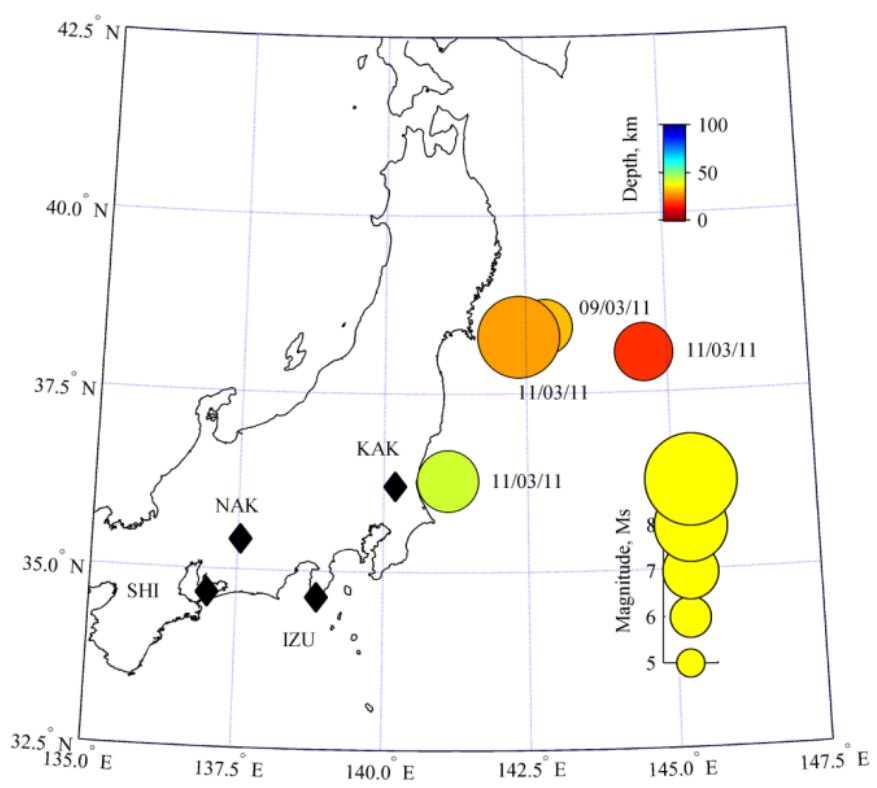

Figure 11: Relative location of our ULF/ELF observing stations (Nakatsugawa(NAK), Shinojima(SHI) and Izu(IZU)) and the epicenter of the 2011 Japan EQ (11/03/11) (biggest circle). For the sake of comparison, one geomagnetic station at Kakioka (KAK) and some other foreshock (09/03/11) and aftershocks (two circles with 11/03/11).After Ohta et al., (2013).

At each observatory we measure the magnetic field changes (H, D and $\mathrm{Z}$ components) by means of three orthogonal magnetometers in the frequency range of $0.1-24 \mathrm{~Hz}$. The magnetometer is an induction coil sensor (similar to those used in Kamchatka), and the receiver attained a high sensitivity of about 0.05 $p T / \sqrt{\mathrm{Hz}}$ at the frequency of $10 \mathrm{~Hz}$. The details of the equipment are described in Hata et al., (2010). The data observed at each observatory are regularly sent to the master station of Chubu University (at Kasugai).

Horizontal components of magnetic field are digitized at the sampling frequency of $100 \mathrm{~Hz}$ with the use of the 16-bit DAS and those data are stored on a hard disk. Those data are transmitted to the master station through a telephone line or an internet.

Here we describe the procedure of ULF/ELF magnetic field analysis in order to detect any seismatmospheric electromagnetic radiation and to determine the azimuth of its source, though some parts are repetitions of previous section.

However, the preliminary routine data processing was applied before the main analysis. That is, this process includes substituting the interpolated data for short (several points) data gaps leading to some errors in DAS, band-pass filtration by means of a 4-order Butterworth filter with cut-off frequencies 0.1 
and $24 \mathrm{~Hz}$. Two-directional filtration was applied to prevent a time shift of data. We take exactly the same analysis procedure used in the previous section dealing with the Kamchatka data.

\section{( ii ) Observational results}

Figure 12 illustrates the result of the spectrum $\Delta S(f)$ at a particular station of NAK during a period from March 4 to March 9, 2011, covering the date of a huge foreshock on March 9. First, we look at the results in the top rectangular panel. The top panel indicates the local seismicity index $\left(K_{S}\right)$.
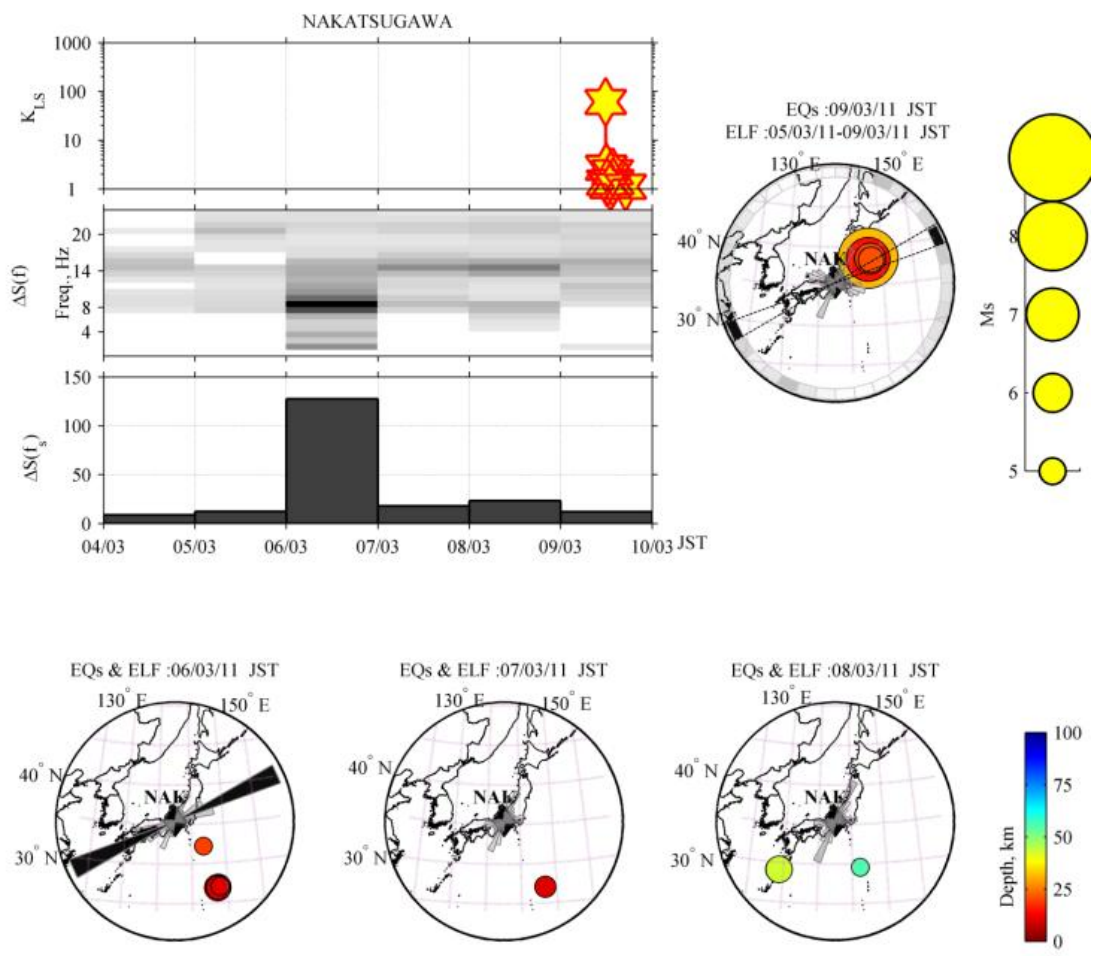

Figure 12: Detection of ULF/ELF radiation at NAK and determination of azimuth of the source before the 2011 Japan EQ. The top plot of the top left rectangular plot illustrates the relationship of local seismicity index $\left(K_{S}\right)$ with $\Delta S(f)$ and $\Delta S(f s)$. The second panel illustrates the temporal evolution of frequency spectrum of $\Delta S(f)$ (more black for more intensity) and the bottom panel indicates the temporal evolution of $\Delta S\left(f_{s}=9-10 \mathrm{~Hz}\right)$ during the period of March 4 to March 9. The top right panel illustrates the overall azimuth plots during the period of March $5-$ March 9, together with EQs with $\mathrm{M}>5$. The bottom three panels illustrate the polar plot of azimuth distribution of ULF/ELF radiation on March 6, March 7 and March 8, respectively (from left to right).After Ohta et al., (2013). 
In the top panel of Figure 12, there is one day of higher $K_{S}$ : a foreshock on March 9 (Ms=7.3) (09/03/11 in Figure 11). In the analysis we have used only the local nighttime data in the JST from 0.5 to $5.0 \mathrm{~h}$ (total duration of 4.5 hours) where we expect the minimum local noise. The middle rectangular panel illustrates the temporal evolution of frequency spectrum of $\Delta S(f)$, in which stronger intensity is indicated with darker black. We can find that on March 6 there is a remarkable enhancement of $\Delta S$ at the frequency of 9$10 \mathrm{~Hz}$. This result is illustrated on the bottom rectangular panel where we show the temporal evolution of $\Delta S$ in this frequency range.

Here we comment on the effect of magnetic storms on the behavior of $\Delta \mathrm{S}$. Although this point was already discussed in our previous papers (Hayakawa et al., 2013a), we repeat only the essential point here. Though the temporal evolution of $D s t$ index as a measure of geomagnetic activity is not shown here, we know that the time of a minor magnetic storm is not coincident with our peak in $\Delta S$ and also the value of $D s t$ is close to null on March 6 when $\Delta S$ is maximal (Hayakawa et al., 2013a). So our peak of $\Delta S$ is very likely to be seismogenic.

Figure 13 is the comparison of $\Delta S$ at the three observatories of NAK, SHI, and IZU during a much longer period of February 1 to March 14, 2011. It is found from this figure that $\Delta S$ at all three observatories exhibits sharp maxima on the same day of March 6, which are 3 days before the March 9 first strong foreshock and 5 days before the March 11 huge main shock. The peak at NAK is conspicuously enhanced because of lower electromagnetic noise there, and the sharp peak on March 6 is still very remarkable at SHI. The electromagnetic noise environment at the third station of IZU is not so good enough that we expect a lot of fluctuations in the variation of $\Delta S$. As the conclusion, ULF/ELF radiation detected by means of $\Delta \mathrm{S}$ appeared 3 days before the first foreshock, which is indicative of the beginning of seismic activity. This is in agreement with the conclusions of our previous statistical studies and gives us a possibility to estimate the time of a forthcoming EQ. Next we determine azimuthal distributions of the radiation. An example of their presentation is shown on the round panels of Figure 12. The distribution of $\alpha$ is represented by an angle histogram, which is a polar plot (as in the bottom circular panels of Figure 12) presenting the distribution of $\alpha$ values. Each group in each polar plot is shown as one bin, and each polar plot shows $\alpha$ (i) in 36 angle bins. The length of each lobe in the histogram and its degree of darkness is proportional to the number of elements in $\alpha$ (i) that fall within a bin. Examples of daily plots on three days (March 6, March 7 and March 8) are given in the bottom three panels of Figure 12. A summary of azimuthal distributions of ULF/ELF radiation for the last 5 days of observations is 
shown by a degree of blackness on the ring which is placed on the top right panel of Figure 12. Its most dark sectors are found to roughly coincide with the azimuths of probable forthcoming EQs. Their limits of probable errors are shown by dashed lines which cross the point of observation.

The azimuthal distribution of ULF/ELF radiation recorded on March 6 when the seismogenic ULF/ELF is strongest is shown on the map of our interest in Figure 14. The size of lobes and degree of blackness as well as in Figure 12 are proportional to the pulse flux density of ULF/ELF radiation. There are illustrated positions of observatories (NAK, SHI, IZU) and EQs with M>7 occurred from March 6 to March 11, and the magnitude and depth of EQs are represented by size and color of circles. Despite of a long scattering of seismic disturbance, the maximum of azimuthal distributions are found to be roughly directed to the epicenter of the EQ.

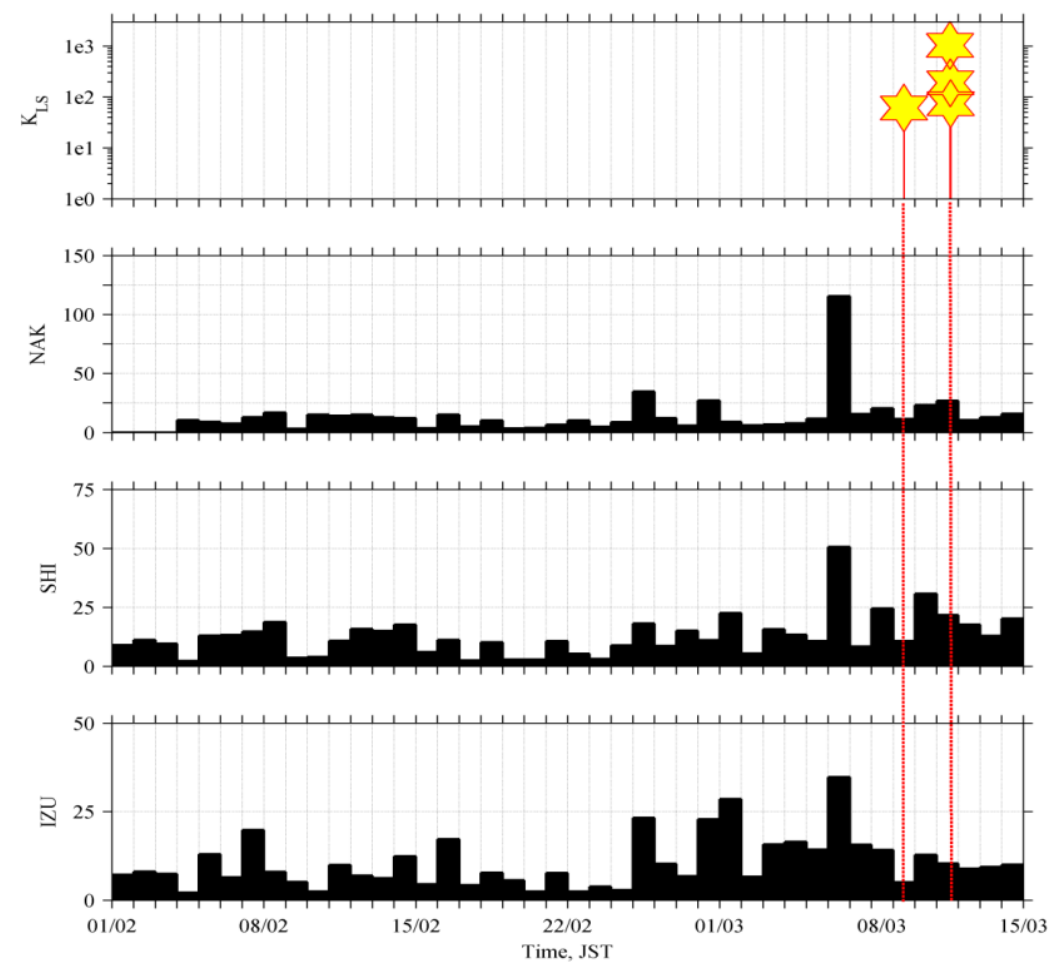

Figure 13: Temporal evolution of $\Delta S(9-10 \mathrm{~Hz})$ at three observatories of NAK, SHI and IZU during a longer period of February 1 to March 14. After Ohta et al., (2013). 
By using the ULF/ELF data in a frequency range of 0.1-24 Hz, we have examined whether there is observed any ULF/ELF precursor to the 2011 March $11 \mathrm{EQ}$. The following facts have emerged from the present analysis, which is a kind of reconfirmation of the previous summary in section 2.2.

1) The combined characteristic of the magnetic field, $\Delta S$ is again proved to be extremely useful in finding out seismo-atmospheric ULF/ELF radiation.

2) The temporal evolution of $\Delta S$ is found to be peaked on March 6, which happened 3 days before the first foreshock which is indicative of the beginning of seismic activity.

3) The frequency of the maximum $\Delta S(f)$ is observed in the vicinity of the first SR.

4) The azimuthal distribution of ULF/ELF radio emission is found to be coincident approximately with the position of the main shock region.

Consequently, we can come to the conclusion that the ULF/ELF radio emissions are highly likely to be generated as a precursor to the 2011 March 11 huge EQ.

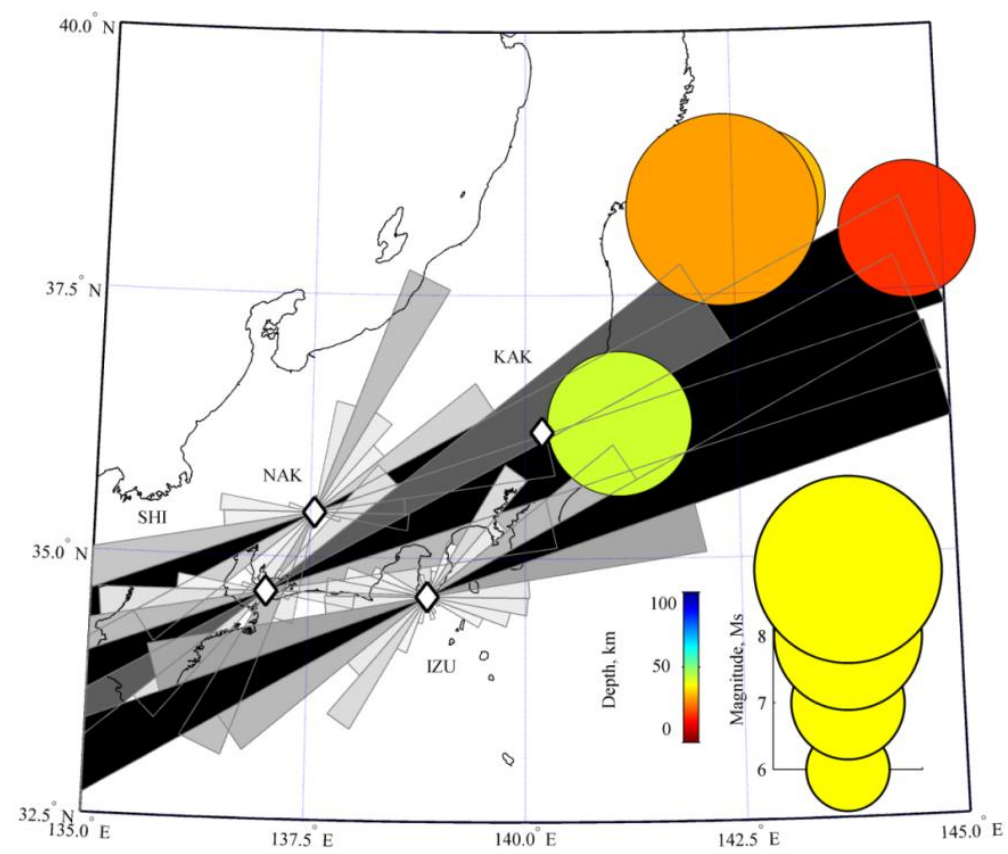

Figure 14: Azimuthal distributions of ULF/ELF radiation recorded on March 6 at three observatories of NAK, SHI and IZU. Positions of observatories are shown by diamonds. Magnitudes and depths of EQs are represented by size and color of circles. After Ohta et al., (2013). 


\section{Anomalies in SR}

This section is intended to review the seismogenic effects in the ELF SR band. Two remarkable anomalies have been found during the last several years in the ELF SR band. The first anomaly in SR was found, as a case study, in the data at NAK in Japan in possible association with a huge EQ (socalled Chi-chi EQ) in Taiwan (Hayakawa et al., 2005) and this kind of SR anomaly has been then confirmed by a statistical study of observation in NAK for EQs with M greater than 5.0 in Taiwan over the period of six years (Ohta et al., 2006). Hayakawa et al., (2008) have further confirmed the presence of similar SR higher mode enhancement at Moshiri, Japan in possible association with another Pingtong EQ in Taiwan, and Ohta et al., (2009) found a new type of anomalies in SR for two Japanese EQs. Recently this topic of anomalous SR effects in possible association with EQs has attracted a lot of attention by different workers in the world (Ouyang et al., 2013; Gazquez et al., 2017; Christofilakis et al., 2019), and future works will provide us with new observational facts.

\subsection{First evidence of anomalous SR phenomena associated with EQs (Case study)}

Three magnetic field components $(\mathrm{Bx}, \mathrm{By}$, and $\mathrm{Bz})$ have been measured at NAK by means of orthogonal induction coil magnetometers (see section 2.3). Bx is the magnetic field in the $\mathrm{N}-\mathrm{S}$ direction, while By that in the $\mathrm{E}-\mathrm{W}$ direction. $\mathrm{Bz}$ is its vertical component. The signal analysis is performed by FFT algorithm with the data length of 1024 , and so the temporal resolution is $10.25 \mathrm{~s}$ and frequency resolution is $0.097 \mathrm{~Hz}(\sim 0.1 \mathrm{~Hz})$. We can estimate the ratio of amplitudes and phase difference oftwo horizontal components among 3 magnetic fields.

Hayakawa et al., (2005) have found the following anomaly in SR observed in Japan (NAK) in possible association with the huge Chi-chi EQ (M=7.3) occurred on 21 September 1999 in Taiwan. Figure 15 is the dynamic spectrum of ELF waves observed at NAK around the time of the EQ, and Figure 16 illustrates the corresponding temporal evolution of the intensity in a specific frequency range of $26.36-26.56 \mathrm{~Hz}$. 


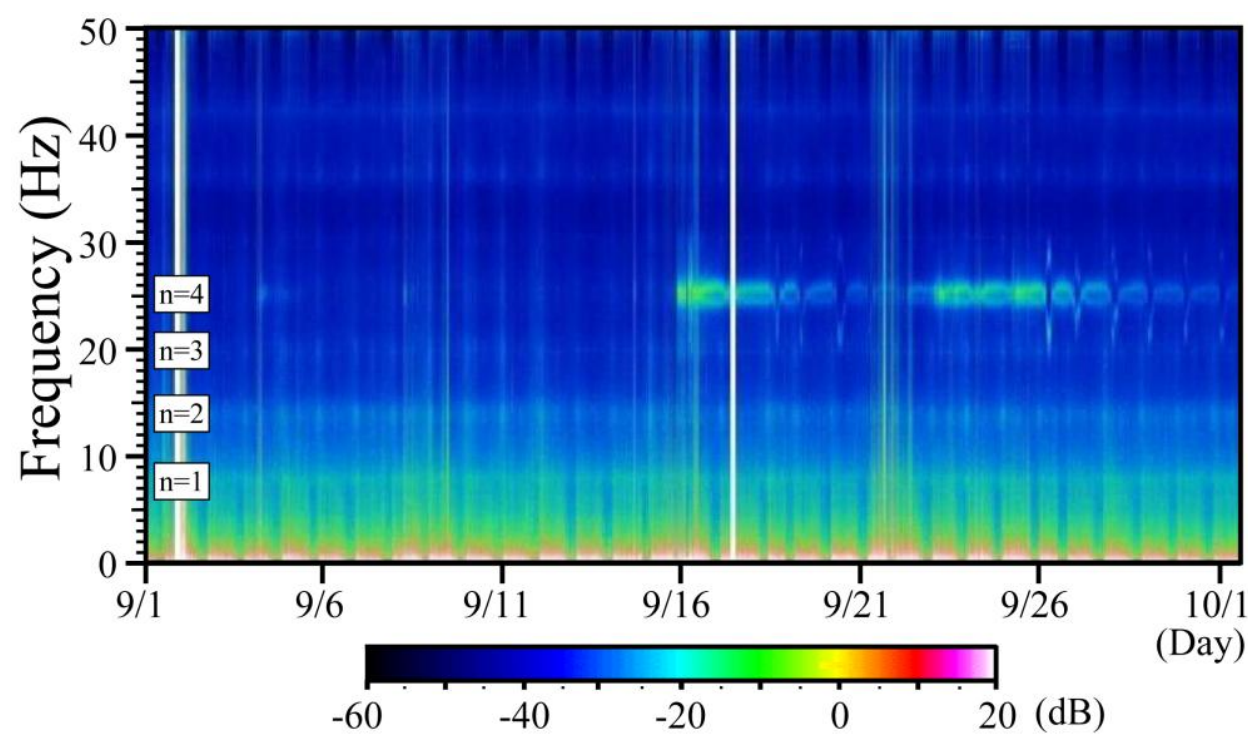

Figure 15: Dynamic spectrum of ELF waves at NAK in the frequency range up to $50 \mathrm{~Hz}$ just around the Chi-chi EQ in Taiwan. The EQ [M 7.6 and depth (D) of $30 \mathrm{~km}$ ] happened at $01 \mathrm{~h} 47 \mathrm{~m} \mathrm{LT}$ on 21 September. We can easily identify an anomaly in SR by comparing the spectra before and after 16 September. The SR fourth harmonic is extremely enhanced before and after the EQ. After Hayakawa et al., (2005) with permission of EGU.

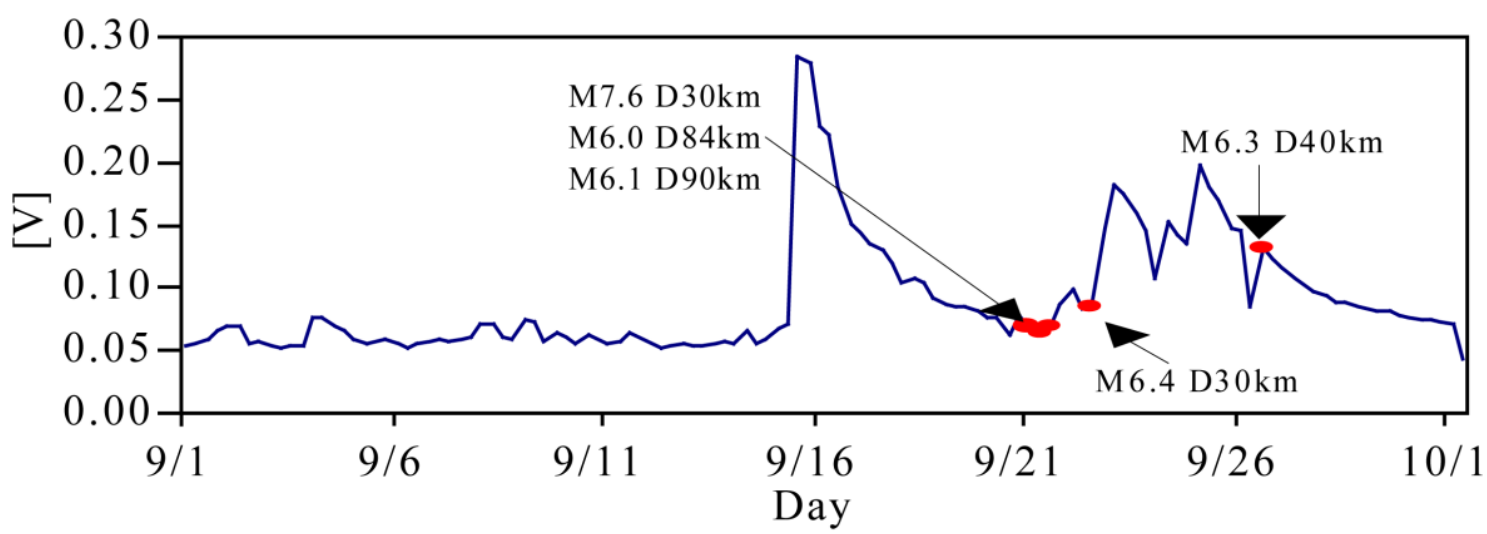

Figure 16: Temporal evolution of the intensity in the frequency range from 26.36 to $26.56 \mathrm{~Hz}$ (about $1 \mathrm{~Hz}$ shifted from the conventional SR fourth harmonic) in relation to the main Chi-chi EQ and subsequent EQs (all aftershocks).

The important findings are summarized as follows from this case study.

(1) The fourth harmonic of the SR is strongly enhanced as the most significant difference from the conventional case. The conventional spectra show that the resonance intensity is decreasing with the resonance number ( $n=1$ is strongest, and the intensity decreases with increasing $n$ ) but the 
anomaly is characterized by the unusual increase in amplitude of the fourth SR mode. This anomaly appears about one week before the EQ.

(2) A significant frequency shift of its peak frequency $(\sim 1.0 \mathrm{~Hz})$ from the conventional value on the By magnetic field component which is sensitive to the waves propagating in the NS meridian plane.

\subsection{Statistical analysis of NAK data (6 years for EQs in Taiwan) and summary}

Ohta et al., (2006) have checked the SR anomaly two weeks before and two weeks after each EQ (with $M$ greater than 5.0). We have used the SR data as observed at NAK during 6 years from the beginning of 1999 to the end of 2004. In Figure 17 there are two types of circles (one is a red circle with white character, and another, a white circle with black character). The first group refers to the EQ event accompanied by the SR anomaly at NAK, while the second group the EQ event without any SR anomaly at NAK.

Summarizing the results in Figure 17, 33 EQs took place in and around Taiwan with $\mathrm{M}$ greater than 5.0 during the six years, but no measurement of SRs could be made for the four EQs in Taiwan. So, we treat $29 \mathrm{EQ}$ events in Taiwan. Among the $29 \mathrm{EQs}$, there are 7 inland (fault type) EQs, and other 22 took place in the sea (oceanic type). The following important points have emerged from the analysis.

(1) The merit of this observation is in rare presentation of a series of anomalous SR data observed in regular fashion when an EQ takes place in Taiwan. This suggests a convincing link between the anomalous SRs at NAK as summarized in section 3.1.and EQs in Taiwan.

(2) All of seven inland EQs are found to have been accompanied with the anomalies in SRs as mentioned in the case study.

(3) Only two from the 22 oceanic EQs, are found to be accompanied by the SR anomaly. These two (No. 9 and 20 in Figure. 20) are examined here. No. 9 had the following peculiarity in the sense that the depth of No. 9 is the shallowest among the 22 EQs, and No. 20 had the largest magnitude among the 22 .

As the conclusion, there is a convincing link between anomalous SR spectra at NAK in Japan and EQs in Taiwan. We can say that the anomalous behaviour in SR is always observed in NAK only for the large and inland EQs in Taiwan. Then, it is found that even the EQ taking place in the sea can trigger the anomalous SR when its magnitude is either extremely large or extremely shallow. 


\section{ULF magnetic field depression phenomenon}

The first report on the depression of ULF horizontal magnetic fields observed on the ground was reported by Molchanov et al., (2003), and later confirmed on the basis of long-term observation (Schekotov et al., 2006, 2013; Schekotov and Hayakawa, 2017). This phenomenon is characterized by the depression (or depletion) of the horizontal magnetic field components of ULF waves (mainly irregular pulsations (Pis) at night). Irregular pulsations (Pis) are one typical type of ULF geomagnetic pulsations, because geomagnetic pulsations are classified from their spectra and waveforms into two types: continuous pulsation (Pc) and irregular pulsation (Pi) (Saito, 1969; Jacobs, 1970; Nishida, 1972). These Pis are observed at night and in the frequency band of $0.01-0.1 \mathrm{~Hz}$ nearly in the same frequency range of the lithospheric ULF radiation (Hayakawa et al., 2011a; Hattori, 2013).

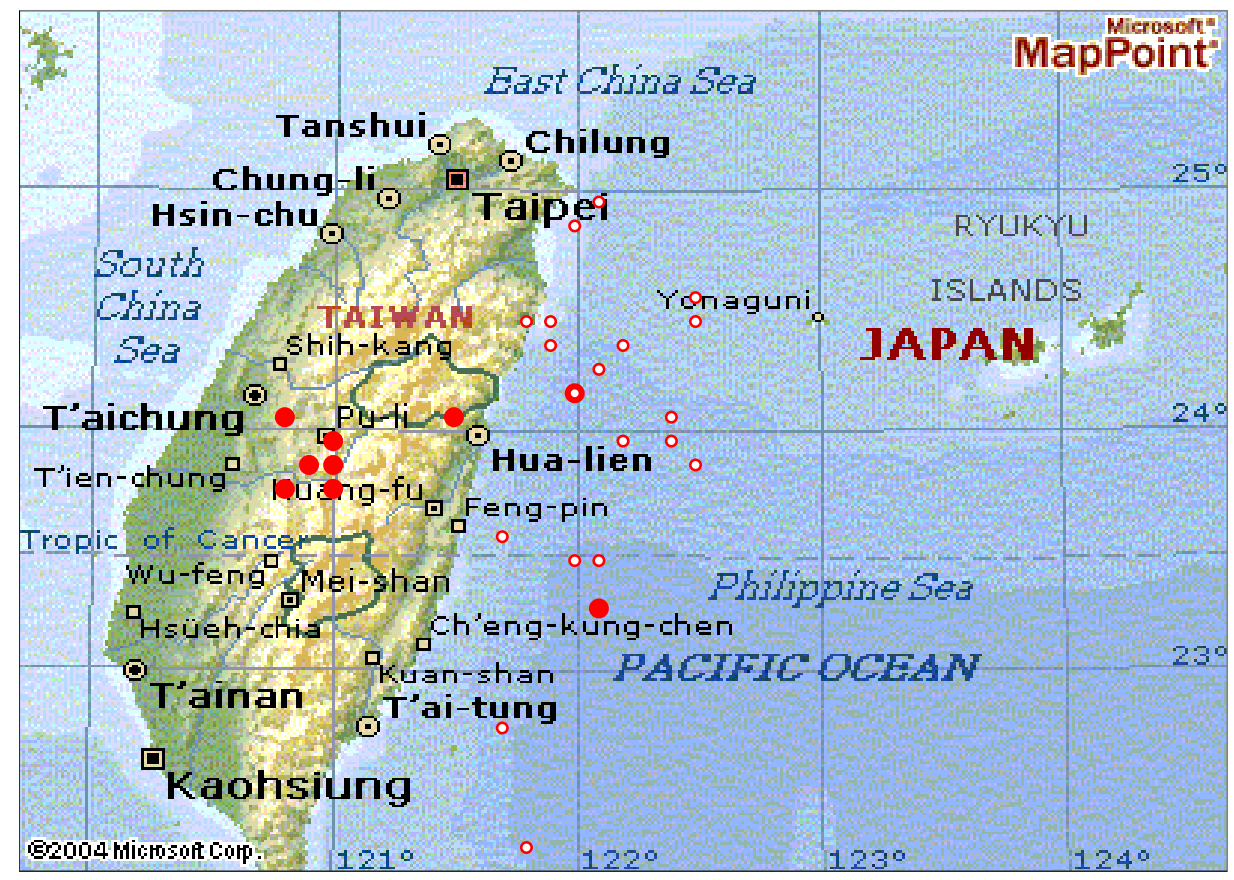

Figure 17: Location of EQs (with $M$ greater than 5.0) in and around Taiwan. Red circle with white character (EQ event number) indicates that this EQ is accompanied by the SR anomaly at NAK. White circle with black character refers to the event without SR anomaly at NAK.Reproduced from Ohta et al., (2006) with permission from Elsevier. 


\subsection{Initial case study}

An example of our initial data from the observatory, Karymshino is presented in Figure 18, which illustrates the temporal evolutions of the spectral density of magnetic field fluctuations during two months in the seven frequency bands from $0.003 \mathrm{~Hz}$ to $5.0 \mathrm{~Hz}$. $\mathrm{Z}$ is the vertical component $\left(\mathrm{P}_{\mathrm{zz}}\right)$ and $\mathrm{G}$ is the total horizontal magnetic field $\left(\mathrm{G}=\mathrm{P}_{\mathrm{hh}}+\mathrm{P}_{\mathrm{dd}}\right)$. You can notice a correlation with $\mathrm{Kp}$ index (magnetic activity) and an evident daily variation, especially in the channels 1,2 and 3. But, a clear signature of correlation with Ks (seismic activity) has not been seen in such a type of analysis.

In order to identify seismogenic ULF emissions from the ULF data, Hayakawa et al., (1996 b) have proposed the use of polarization, which is reduced to the analysis of ratio of $\mathrm{Z} / \mathrm{G}$ in our research. The results for the first time interval of 7 months (from June 26, 2000 to February 25, 2001) are shown in Figure 19. In contrast with Figure 18, some correlation with Ks can be supposed at least for the frequency channels 2 and 3 and near the date of large Ks values (these cases are indicated by vertical dotted lines). In order to check it we demonstrate all the cases in Figure 20, each case in the vicinity of the five most powerful EQs. Relying upon the results in Figure 19 and for simplicity we present only channel 2 $(\mathrm{f}=0.01-0.03 \mathrm{~Hz})$. It is obvious that nighttime values of $\mathrm{Z} / \mathrm{G}$ exhibit an increase about 2-7 days before each EQ date.

An important question arises immediately: what does an increase of polarization mean? Is it due to an increase of $\mathrm{Z}$ component or a decrease of $\mathrm{G}$ component or both? Figure 18 shows that these change practically synchronously. But because the value of $\mathrm{Z}$ is about an order smaller than $\mathrm{G}$ while they decrease together, $\mathrm{Z}$ quickly reaches a noise level of the magnetic sensor or its reduction is limited by the interference, and then the polarization is likely to depend only on G. To confirm this assumption we present $\mathrm{Z} / \mathrm{G}$ values and 1/G values after 1 day averaging in Figure 21 for the same case as in Figure 20. Figure 22 illustrates the cross-correlation function of the 2-day averaged local seismicity Ks and variation of ULF depression $\delta(1 / \mathrm{G})$. Here

$\delta(1 / \mathrm{G})=(1 / \mathrm{G}-\langle 1 / \mathrm{G}\rangle) /\langle 1 / \mathrm{G}\rangle$ and $\langle 1 / \mathrm{G}\rangle$ is one month running mean value. This dependence shows that the depression maximum reliably exists before an EQ by about 2-4 days. This was the first result of a study of this phenomenon reported by Molchanov et al., (2003). 


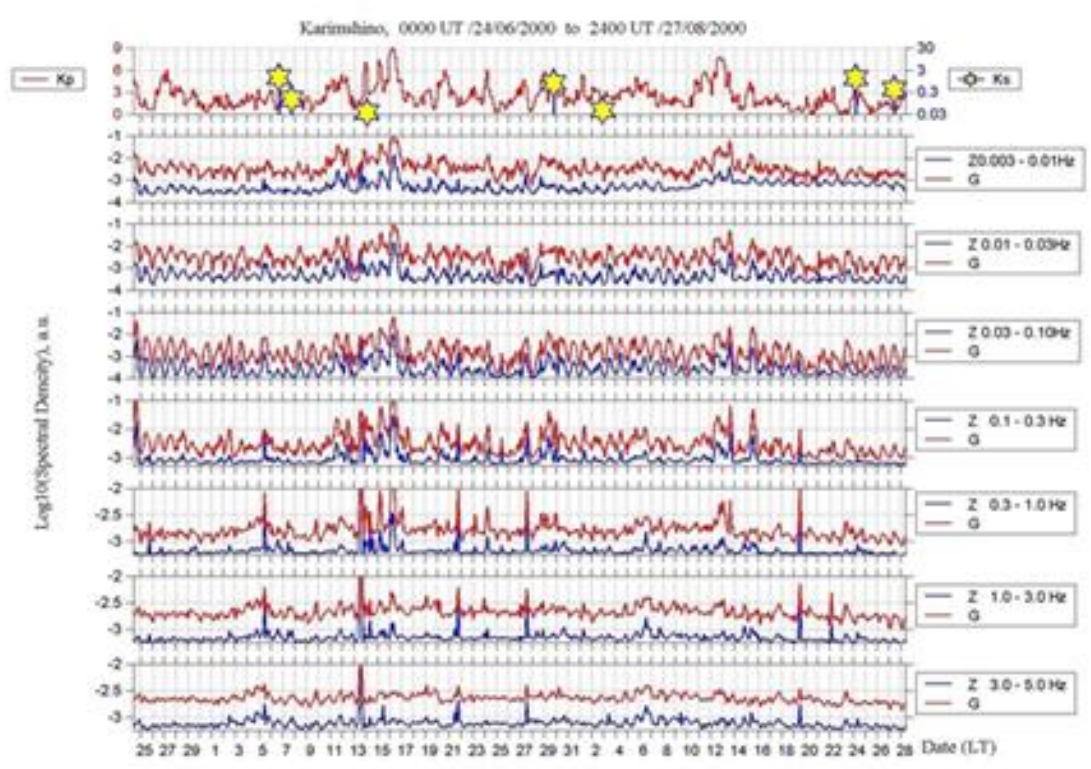

Figure 18 :( Top panel) Indices of seismic activity Ks (yellow stars) and global magnetic activity Kp in the upper panel. Examples of ULF magnetic field variations at 7 filter bands during 2 months of observation in Karymshino are shown in the subsequent panels. $Z=P_{z z}$ indicates the intensity of vertical component and $G$ is the intensity of horizontal components, $\mathrm{G}=\mathrm{P}_{\mathrm{hh}}+\mathrm{P}_{\mathrm{dd}}$. After Schekotov et al., (2013) with permission from TERRAPUB.

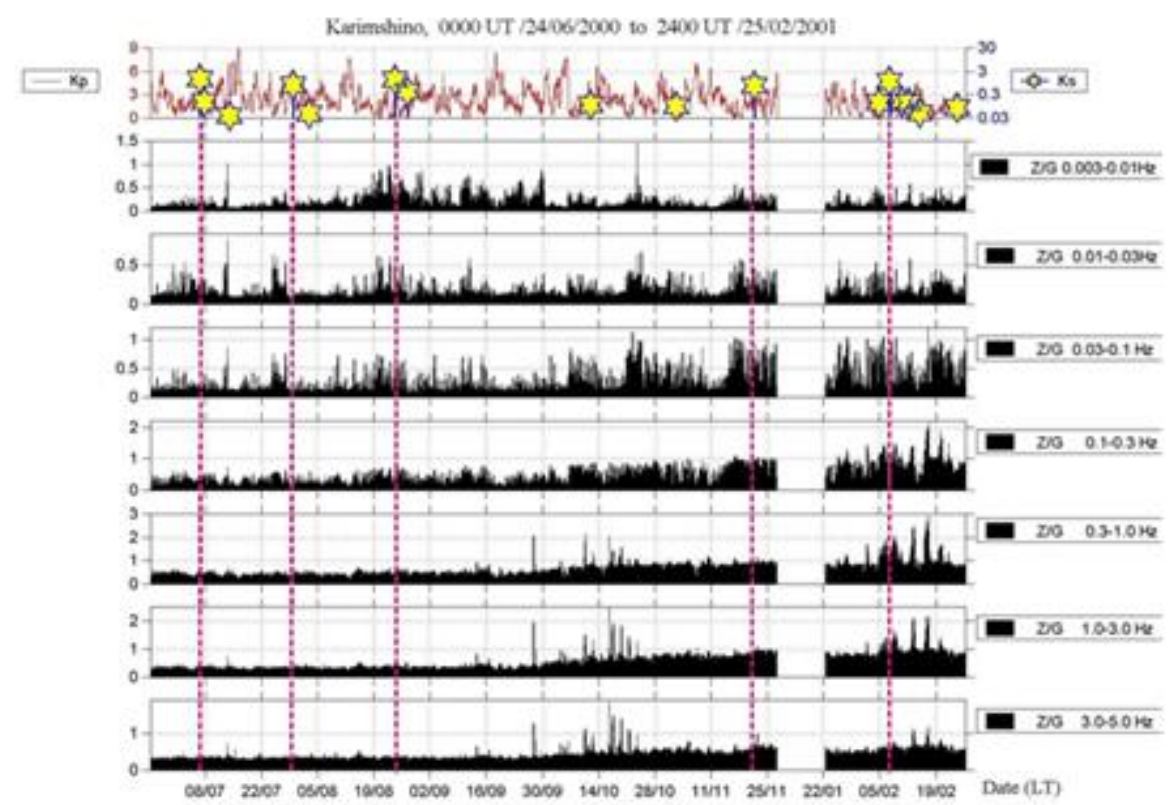

Figure 19: Ks, $\mathrm{K}_{\mathrm{P}}$ (top panel) and polarization $\mathrm{Z} / \mathrm{G}$ in the different frequency ranges (subsequent 7 panels) during 7 months of observation at Karymshino station. After Schekotov et al., (2013) with permission from TERRAPUB. 


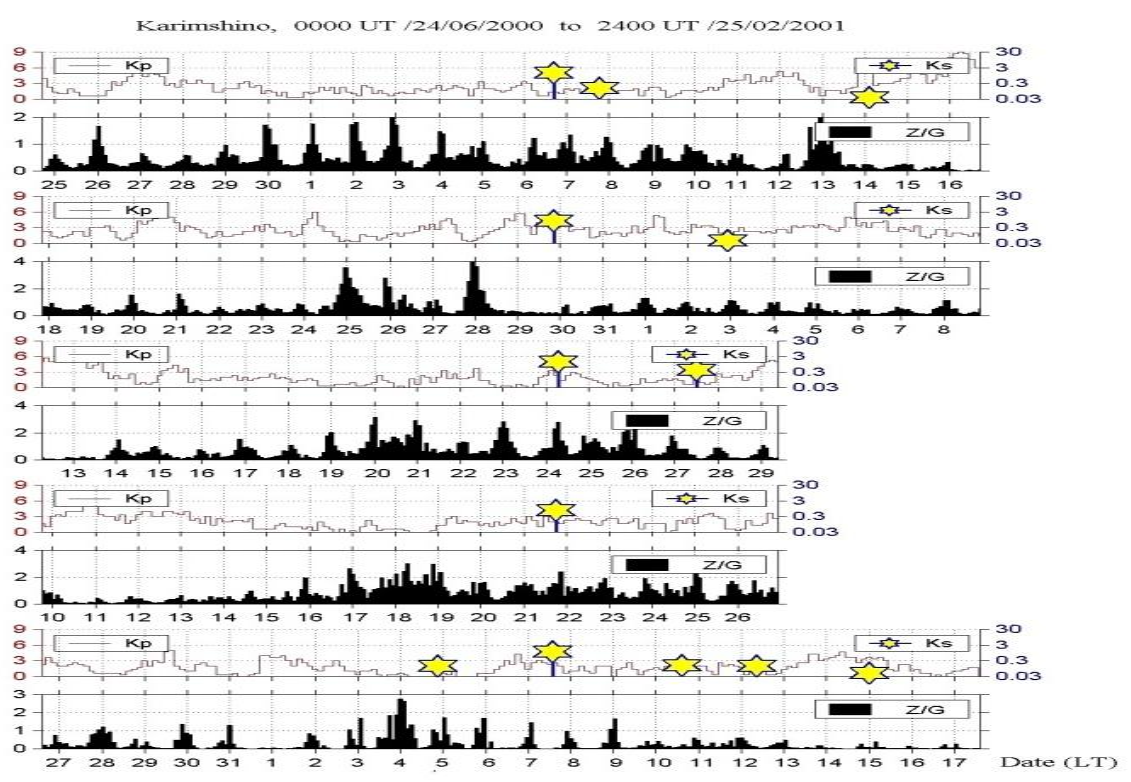

Figure 20: Ks, Kp and polarization in the vicinity of the five most powerful EQs in the interval from 24/06/2000 to 25/02/2001. After Schekotov et al., (2013) with permission from TERRAPUB.

a)

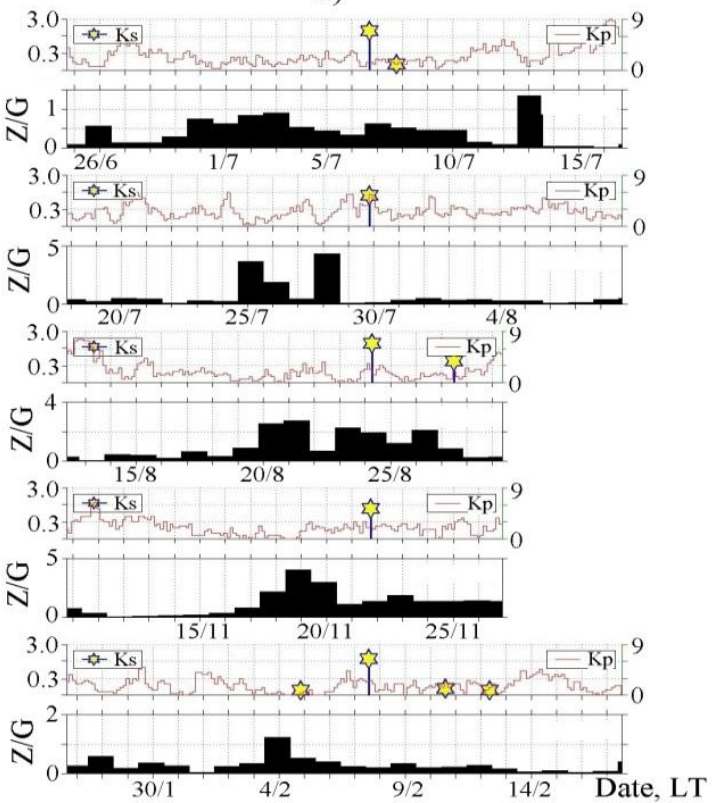

b)

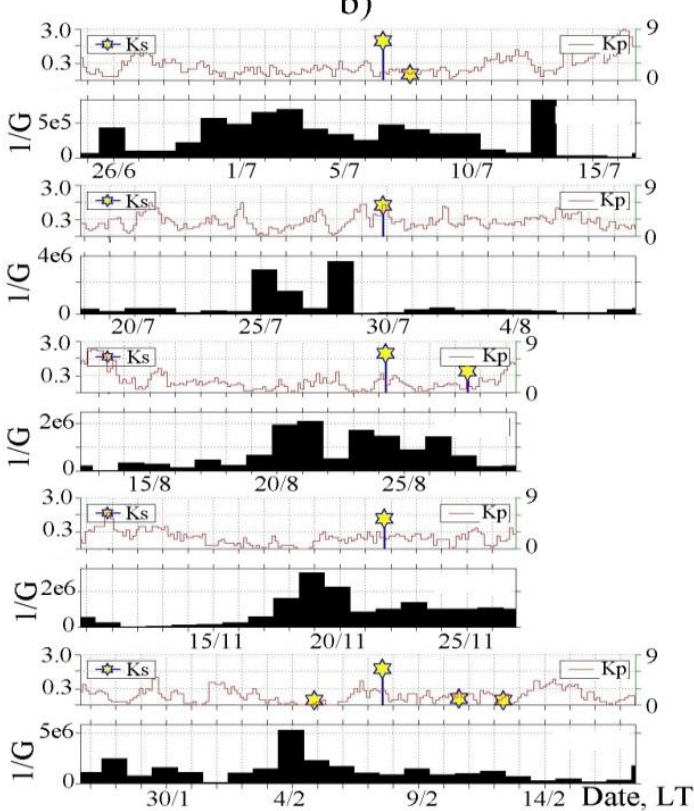

Figure 21 (a): The ratio of Z/G around the same EQs as in the previous Figure after 1-day averaging. (b) The same as (a) but for $1 / \mathrm{G}$ values and in the corresponding scale. After Schekotov et al., (2013) with permission from TERRAPUB. 


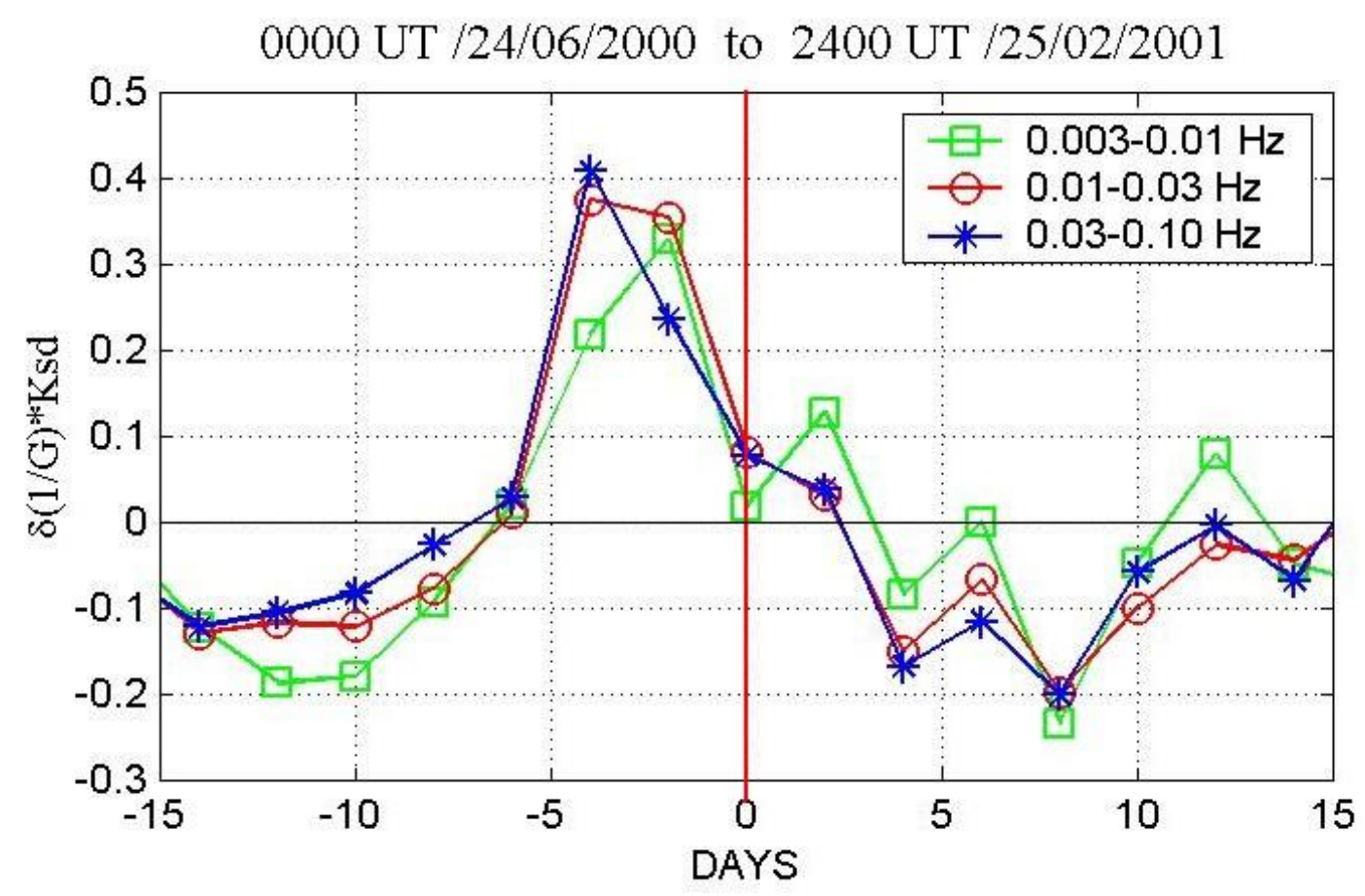

Figure 22: Cross-correlation function of the 2-day averaged local seismicity Ks and variation of the depression $\delta$ $(1 / G)$ at different frequency bands. After Schekotov et al., (2013) with permission from TERRAPUB.

\subsection{Statistical result}

This statistical study is based on Schekotov et al., (2006). Because we have accumulated the data for the period from June 2000 to June 2004, we were interested in whether we will see this phenomenon in the following three years of observations. If so, it is crucial for the short-term EQ prediction whether this effect appears 2-4 days prior to an EQ. We were interested as well in the dependence of ULF depression on parameters of local seismic activity and the possibility of observing this phenomenon in other regions as well. At this stage we have defined a relative variation of depression $\delta \mathrm{D}$, and the cross-correlation functions $\delta D^{*} \mathrm{Ks}$ are calculated.

Because the effect is uncertain for EQ series, only isolated EQs are chosen for the analysis. We did not consider EQs with $\mathrm{Ks}<1$ and aftershocks in the interval less than one week after the main shock. As a consequence the number of isolated EQs or the first in a series with $\mathrm{Ks} \geq 1$ reduces to 39. One example is illustrated in Figure23. Here is an example of variation of the field depression $\delta \mathrm{D}, \mathrm{Kp}$ and $\mathrm{Ks}$ on the topleft panel and a map (top-right) with parameters of corresponding EQ which happened on 16/06/2003. 
Bottom panels are depression spectra for local noon (red) and midnight (blue). Maximum night-time depression occurs on the night of June 13, 3days before the EQ. Especially the depressed intensity belongs to the frequency range of $0.02-0.05 \mathrm{~Hz}$, while black line shows the inverse sensitivity of the magnetometer.
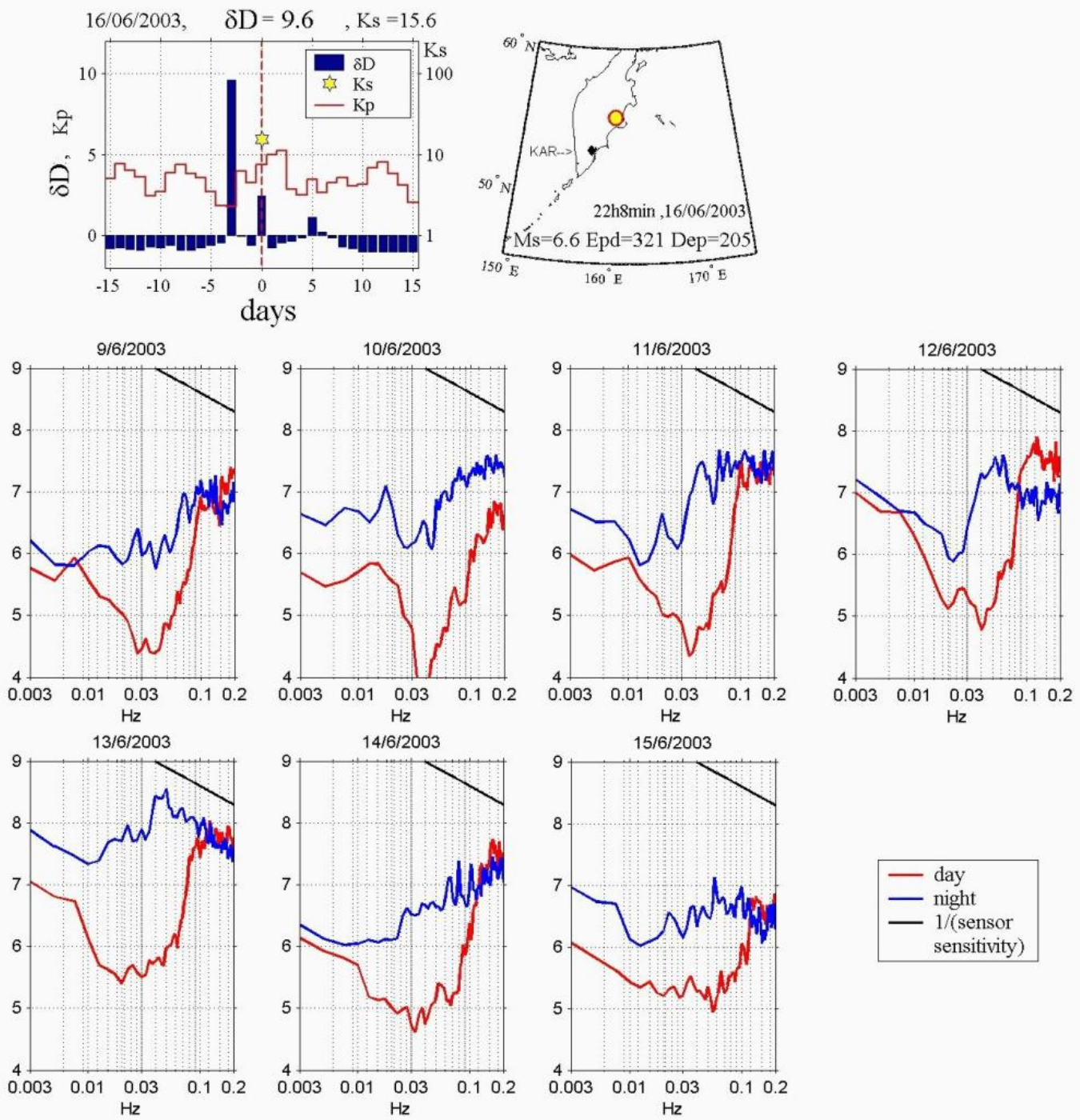

Figure 23: Top panels are an example of variation of the field depression $\delta \mathrm{D}, \mathrm{Kp}$ and $\mathrm{Ks}$ and a map with parameters of the corresponding EQ. Bottom: depression spectra for local noon (red) and midnight (blue) for the seven days. After Schekotov et al., (2013) with permission from TERRAPUB. 
The cross correlation function $\delta \mathrm{D} * \mathrm{Ks}$ between $\mathrm{Ks}$ index and depression of the magnetic field $\delta \mathrm{D}$ is shown on the left panel of Figure 24, and the averaged variation of depression by the SPE method is shown on the right panel. Both curves are found to have a clear maximum 3 days before an EQ.

In order to validate the statistical stationarity of the effect the interval of observations is divided into 4 one-year intervals and the cross correlation functions are calculated for each interval (the bottom panel of Figure 25). The clear maximum at 3 days before the EQ is found for the 1, 3 and 4 year intervals as well as for the whole period of observations. As for the second interval, there take place additional maxima comparable with the "3-day" maximum. Thus, no meaningful effect is found for the second interval, but this is probably because of low level of the nighttime geomagnetic activity during the second interval (see the upper panel of Figure 25).
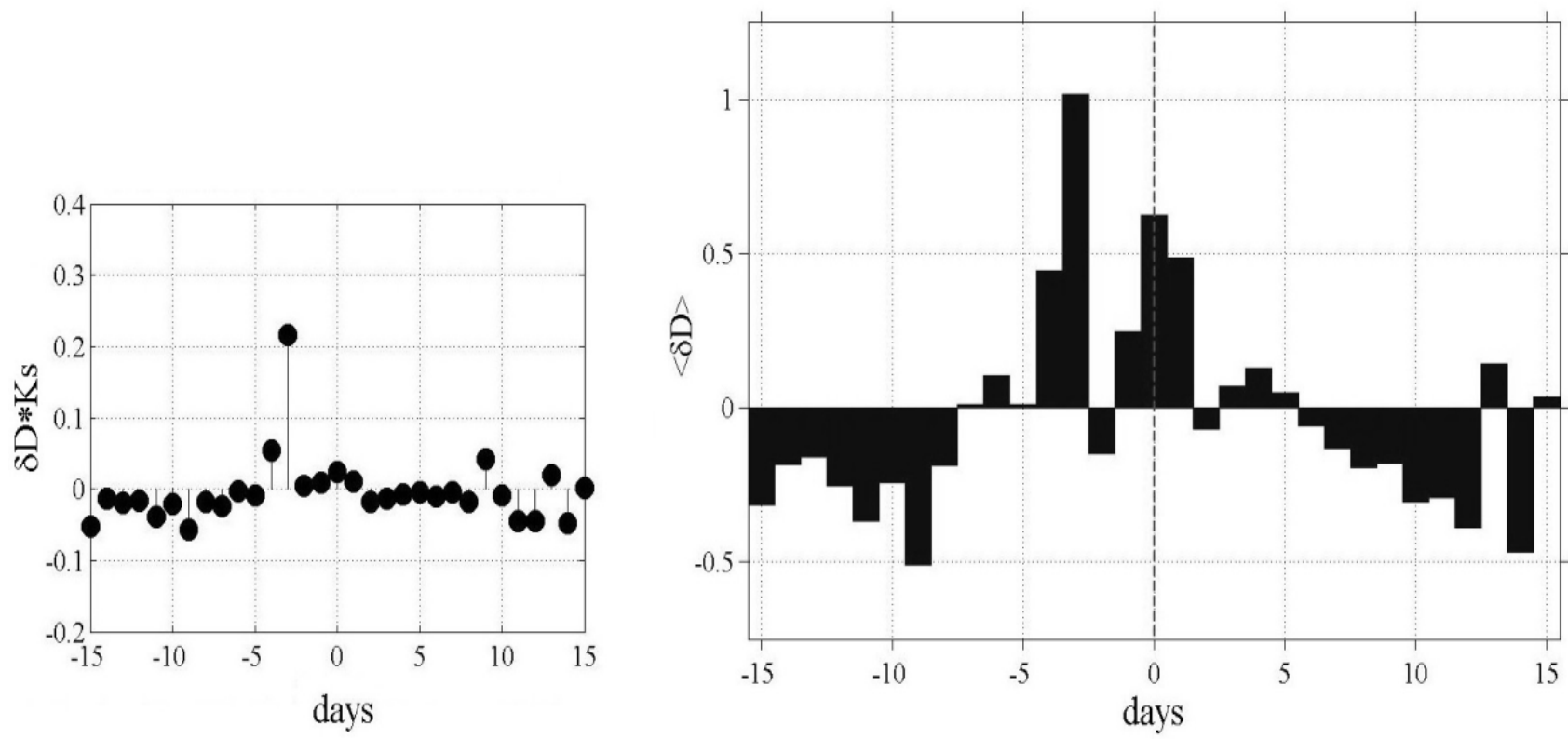

Figure 24: Left: Cross-correlation between Ks of the selected EQs and field depression for the whole period of 4years observation. Right: Variation of the field depression averaged over all the selected EQs by SPE method. After Schekotov et al., (2013) with permission from TERRAPUB. 


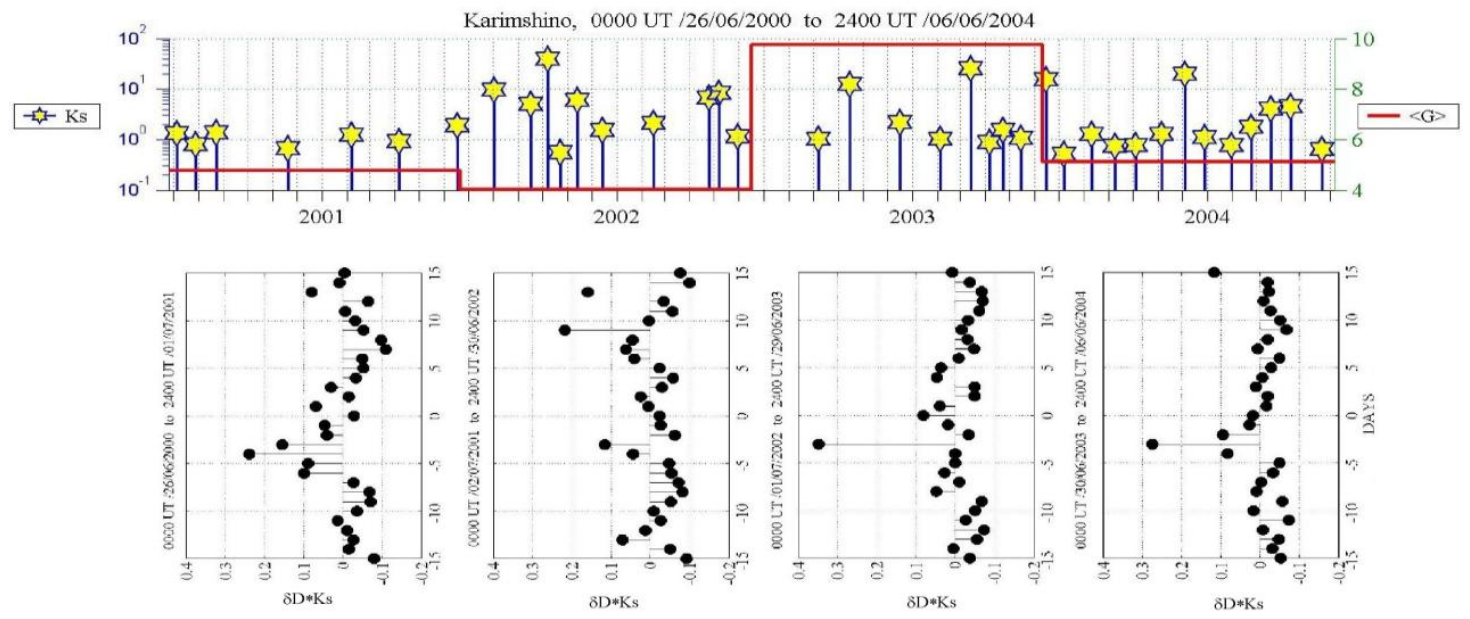

Figure 25: Top: Ks index for EQs and year-mean field magnitude at the frequency range $0.03-0.5 \mathrm{~Hz}$ in the vicinity of \pm 0.5 hour around the local midnight. Bottom: cross-correlation functions for each year interval. After Schekotov et al., (2013) with permission from TERRAPUB.

The dependence of depression on Ks is demonstrated in Figure 26. It can be seen from this figure that Ks dependence becomes more or less clear for EQ magnitude value $\mathrm{M}>5$ and the correlation seems to be improved for EQ depth less than 50km.
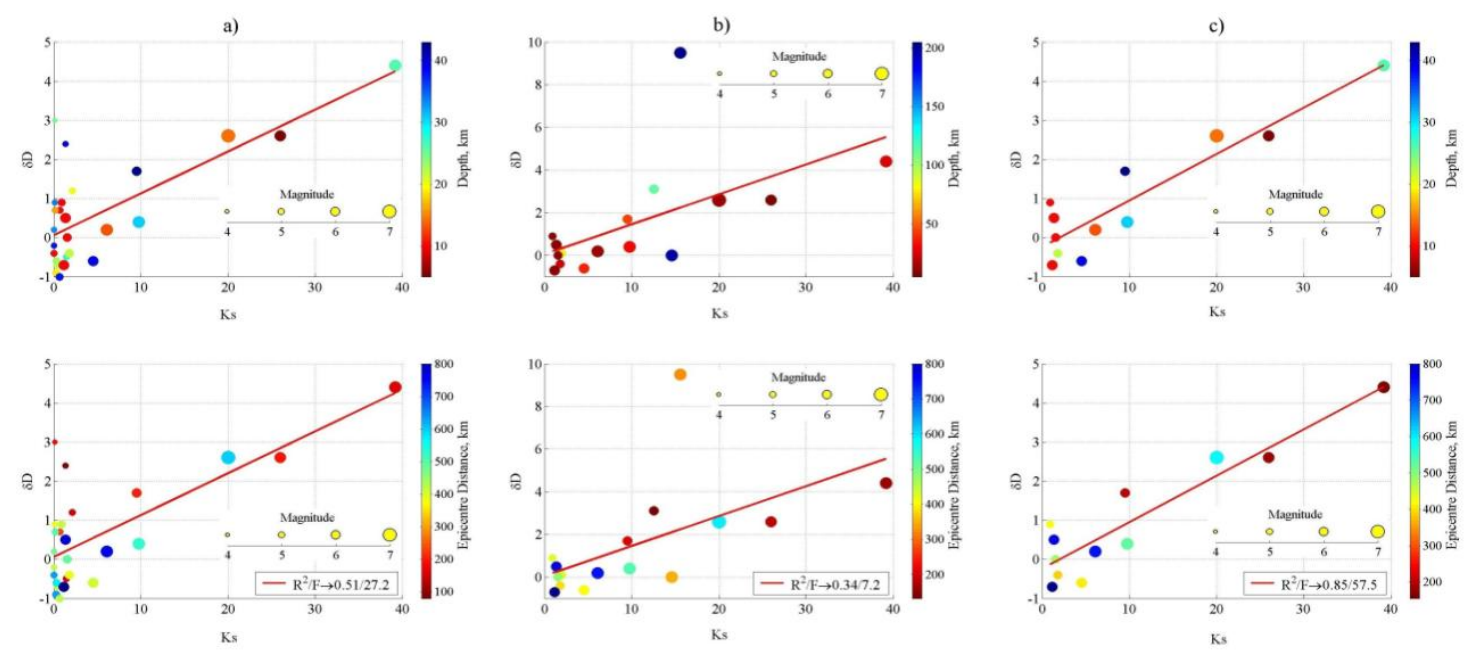

Figure 26: Depression $\delta \mathrm{D}$ vs Ks. (a) for $\mathrm{Ms}>4$ and depth $<50 \mathrm{~km}$, (b) for $\mathrm{Ms}>5.5$ and depth $<300 \mathrm{~km}$, (c) for Ms $>5.5$ and depth $<50 \mathrm{~km}$. Color of circles shows EQ epicenter depth on the upper panels and epicenter distance on bottom panels and their size depends on the EQ magnitude Ms. After Schekotov et al., (2013) with permission from TERRAPUB. 
Then we try to find the depression effect from the data by a rather sensitive flux-gate magnetometer at the Japanese station Matsukawa $\left(39.88^{\circ} \mathrm{N}, 140.94^{\circ} \mathrm{E}\right)$ using the same technique. The "3-day" depression effect is also found for these data.

At last in order to confirm that the effect is not coincidental and local, we produce the SPE overlapping of Karymshino data for nearby EQs in Matsukawa and vice versa in the same 2-year interval when simultaneous data of both stations are available. The results are shown in Figure27. It is seen from the right panels of Figure 27 that the field depression at Karymshino is not related to Japanese EQs and the field depression at Matsukawa does not depend on seismicity at Kamchatka.
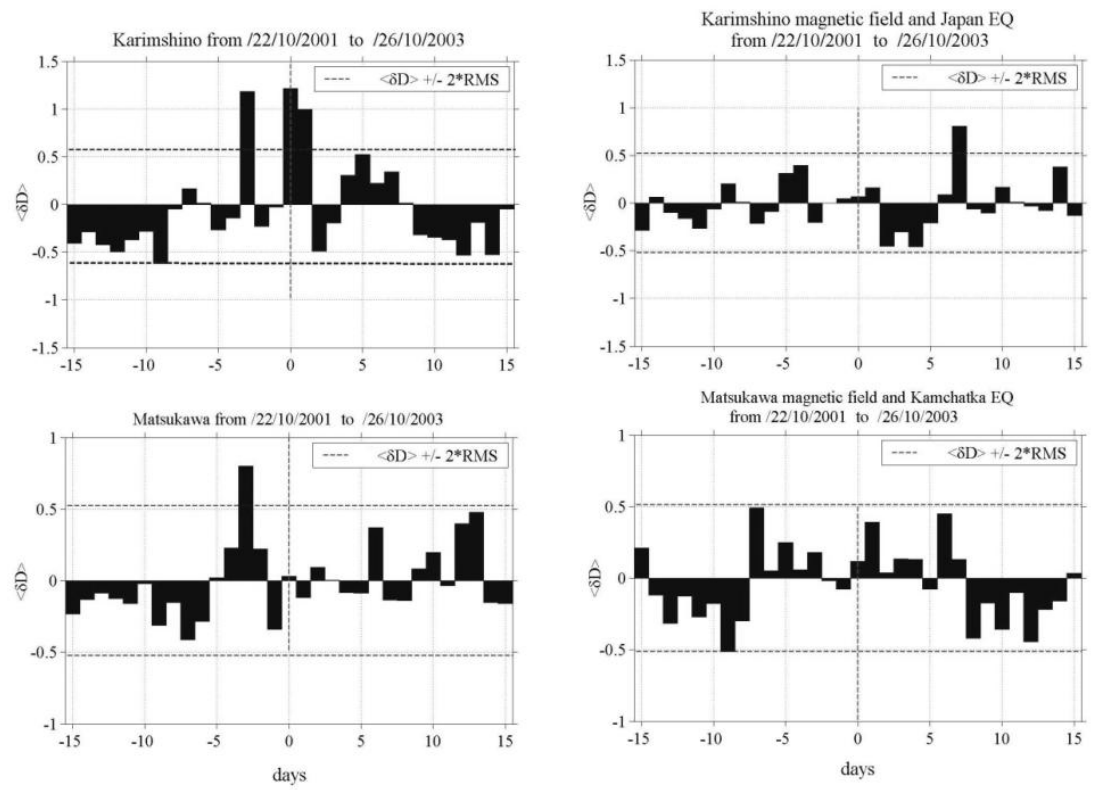

Figure 27 : Left top: Averaged variation of the field depression for Kamchatka; left bottom- for Japan in the same 2-year interval; right top - magnetic field depression at Kamchatka averaged over dates of the nearby Japanese EQs; right bottom - depression at Japan for the nearby EQ dates at Kamchatka. Double dispersion is shown by dashed lines. After Schekotov et al., (2013) with permission from TERRAPUB.

\subsection{Further recent example for the 2011 Tohoku $E Q$}

The ULF depression was observed also for the recent huge Japan EQ (M 9.0) on March 11, 2011, which is a typical oceanic EQ of the plate type (Hayakawa et al., 2013a). Figure 28 is their result of analysis of relative ULF magnetic field depression ( $\delta \mathrm{Dep}$, exactly the same as $\delta \mathrm{D}$ in the previous section)) at three 
Japanese observatories (Memambetsu (MMB) in Hokkaido, Kakioka (KAK) in the main island, and Kanoya (KNY) in Kyushu). The definition of this relative depression is given in Hayakawa et al, (2013 a, b). The top panel indicates the temporal evolutions of Dst and the occurrence of EQs with M greater than 5. The figure shows that remarkable ULF depression is taking place on March 6, 5 days before the main shock at all stations, but the most significant depression is found to be observed at KAK in the main island, which is closest to the epicenter of the main shock, and so this anomaly is considered to be a precursor to this EQ.

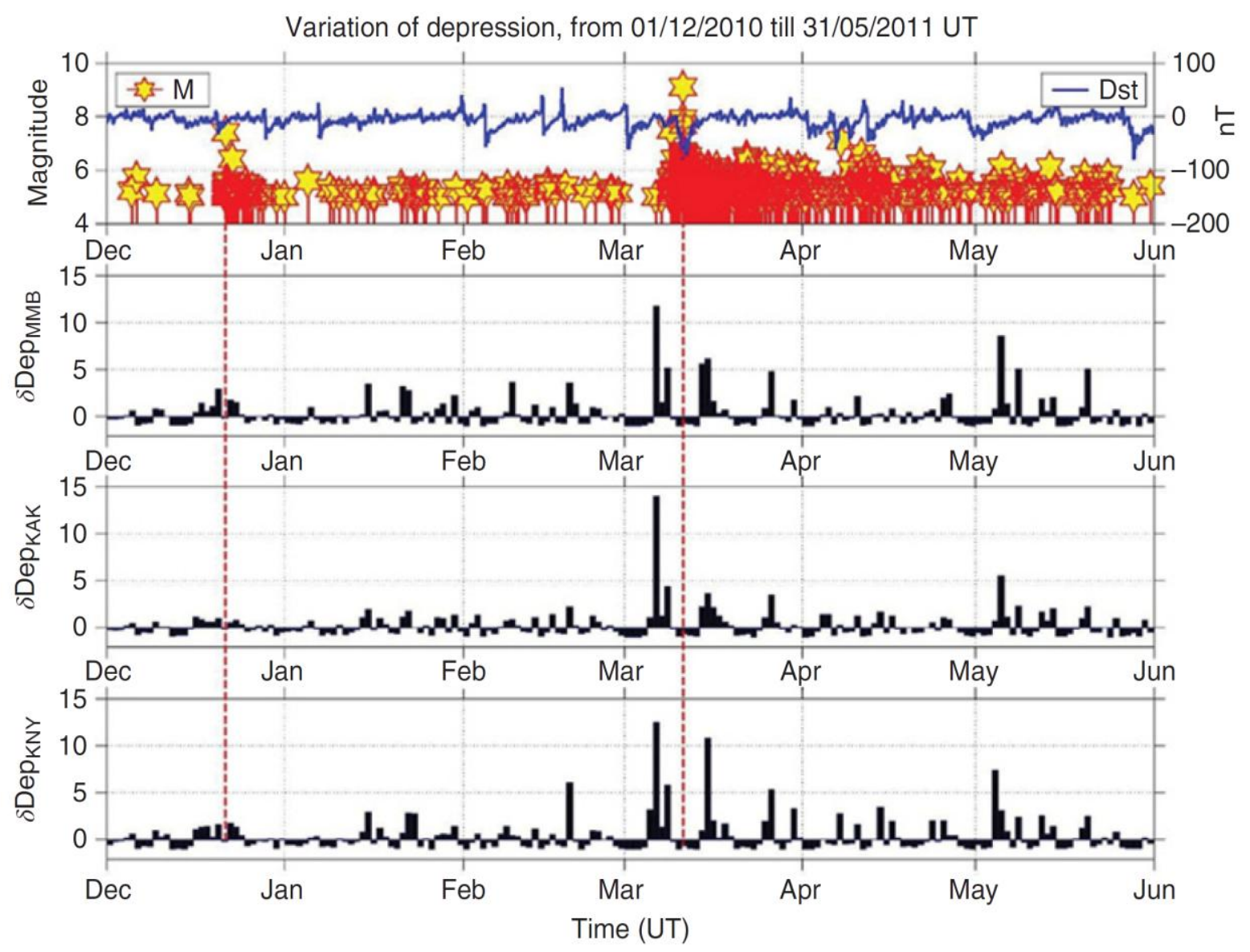

Figure 28: ULF magnetic field depression for the 2011 Japan EQ. The second panel refers to the station of MMB, the third panel, KAK, and the last panel, KNY. After Hayakawa et al., (2013b) with permission from Taylor and Francis. 


\subsection{Summary}

Two additional huge EQs in the Kuril Islands (M 8) have been analyzed, and the quite similar ULF depression for these EQs has been confirmed by Hayakawa et al., (2013b).Further studies have been carried out by Schekotov et al., (2013) and Schekotov and Hayakawa (2017) and we come to the summary of basic properties of the effect of ULF magnetic field depression:

1) About 3 days before an EQ in the vicinity of local midnight there occurs a decrease in horizontal magnetic field fluctuations(irregular pulsations) in possible association with EQs,

2) it is especially noticeable at the frequencies of $0.03-0.05 \mathrm{~Hz}$,

3) the value of depression depends linearly on the local seismicity (or M),

\section{EQ effect on Pc1 pulsations}

There is published the first paper on the statistical relation of Pc 1 pulsations observed at low latitudes with EQs by Bortnik et al., (2008), and we refer to their results.

A simple explanation of Pc 1 pulsations observed at medium/low latitudes is required (e.g., Jacobs, 1970; Nishida, 1972; Alperovich and Fedorov, 2007; Surkov and Hayakawa, 2014). As shown in Figure 29, electromagnetic ion Cyclotron (Alfven) waves are originated in the equatorial plane of the magnetosphere at high latitudes in terms of wave- particle interactions. Those waves propagate roughly along the magnetic field in Alfven (L)mode, and they enter the ionosphere at high latitudes, and they couple to the isotropic magneto sonic $(\mathrm{R})$ mode in the ionosphere and propagate horizontally in the ionospheric waveguide, which is centered on the F2 region electron density maximum at $~ 300 \mathrm{~km}$ (e.g., Manchester, 1966). The wave power progressively attenuates and leaks to the ground through the lossy bottomside ionosphere from high to low latitudes, the attenuation being more severe during the daytime, resulting in a nighttime occurrence maximum at low latitudes (e.g., Saito, 1969; Jacobs, 1970). 


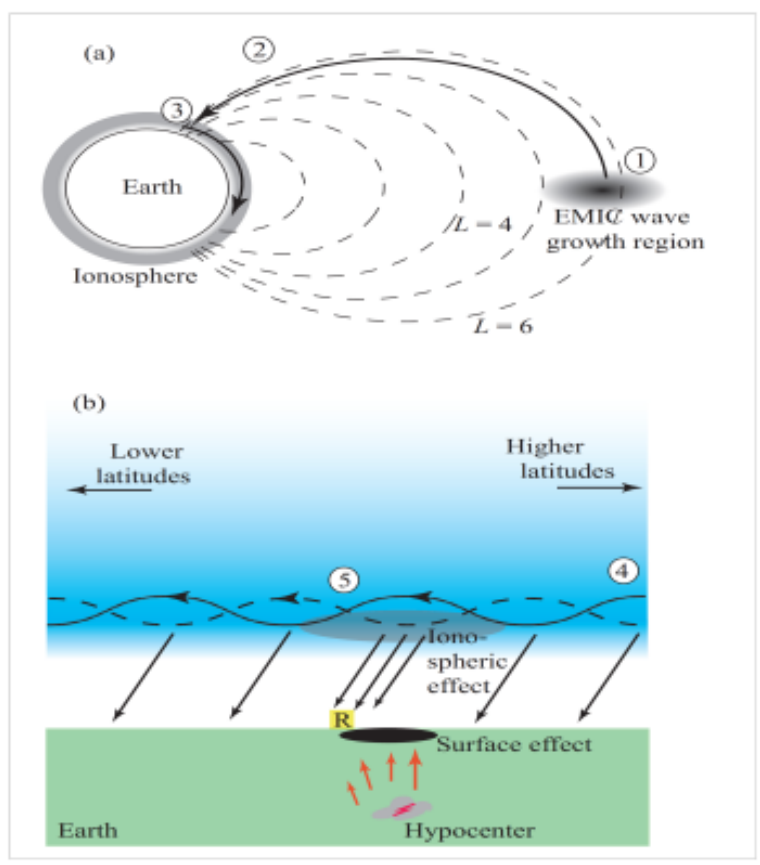

Figure 29: A possible mechanism of Pc 1 modulation by EQs. (a) The electromagnetic ion Cyclotron wave is generated near the equatorial region of the magnetosphere (1), propagates roughly along the magnetic field (2), enters the ionosphere at high latitudes (3), and propagates within the dense F-region of the ionosphere to low latitudes (ionospheric waveguide propagation) (4), and seismically active regions might affect the ionosphere in such a way as to change the bottomside transmission coefficient (5), resulting in anomalous Pc 1 activity recorded at the receiver, R. After Bortnik et al., (2008) with permission from EGU.

The data are obtained at Parkfield, California (geographic coordinates: $35.945^{\circ} \mathrm{N}, 120.542^{\circ} \mathrm{W}$ ) during a long period of 7.5 years from February 1999 to July 2006. The identification of Pc1 pulsations was made by their own algorithm.

In order to examine the association between Pc1 occurrence and EQs, they performed a SPE analysis spanning - 30 days to +10days about the EQ time. They selected EQs whose epicenters were within $200 \mathrm{~km}$ of the ULF station, M larger than 3.0, and depth smaller than 50km. Figure 30 (a) illustrates the local time dependence, and Figure 30(b) is the distribution of EQs as a function of distance and magnitude.

The entire set of identified Pc1 pulsations is binned into 12 LT bins. The resulting histogram is shown in Figure 30(a), which is divided by the total number of days of instrument operation to effectively obtain a long-term, daily occurrence probability of Pc1 pulsations. Similarly, the SPE plot in Figure 30(c) is normalized by the total number of EQ events to obtain a time-dependent probability of occurrence, and 
then each column is normalized by the daily occurrence probability, resulting in a distribution of Pc1 occurrence probability, as a function of $\Delta \mathrm{T}$ (time delay) and LT, relative to the long-term occurrence probability. The resulting plot is shown in Figure30(d), which indicates that the relative probability of occurrence of Pc 1 pulsations at Parkfieldis significantly enhanced during the day time hours, 5- 15days prior to the EQ occurrence.

They have integrated the number of Pc1 pulsations in Figure 30(c) from 08:00 to 16:00, and have shown the resulting line plot in Figure30 (e). Similarly, they have averaged the relative occurrence probability in Figure 30 (d) over the same LT interval and show the line plot in Figure 30 (f). In both panels Figures 30 (e) and 30(f), they have found a clearly evident fact of the increase of Pc1 pulsations during midday, $\sim 5-15$ days before an EQ. We note that this time scale is roughly consistent with that of ionospheric anomalies by previous workers such as Liu (2009) and Hayakawa et al., (2010).

They have performed tests of significance, and Table2 is the summary on the assessment whether the presence of midday Pc1 pulsations might be useful as a reliable indicator of imminent EQs. They have attempted to quantify the number of false positive and negative events. Their results seem to be promising for short- term EQ prediction. Pc 1 pulsations are fundamentally high-latitude phenomena, and so it is very difficult to think that the source region deep in the magnetosphere or source mechanism is likely to be influenced by EQs. The only possible effect of EQs might take place when they propagate in the ionospheric waveguide from high to low latitudes as shown in Figure 29.So, it is highly required that their results can be repeated with data from other periods and especially other locations at different latitudes in the world, in order to verify their results.

Table 2 : Percentage of midday Pc 1 pulsations with and without an EQ ensuring within 2 weeks, and vice versa. Columns correspond to the full EQ catalog $(M \geqq 3$, within $200 \mathrm{~km})$, the mean and standard deviation $\sigma$ of 300 similar random event samples.

\begin{tabular}{lcccc}
\hline & Full & Random 434 $(\mu, \sigma)$ & Declustered & Random 268 $(\mu, \sigma)$ \\
\hline EQ's with Pcl & $63.1 \%(274 / 334)$ & $52.5 \%, 2.5 \%$ & $50.0 \%$ & $52.5 \%, 3.0 \%$ \\
EQ's without Pc1 & $36.9 \%(160 / 334)$ & $47.5 \%, 2.5 \%$ & $50.0 \%$ & $47.5 \%, 3.0 \%$ \\
Pcl with EQ's & $72.6 \%(307 / 423)$ & $87.7 \%, 3.6 \%$ & $72.6 \%$ & $75.6 \%, 5.1 \%$ \\
Pcl without EQ's & $27.4 \%(116 / 423)$ & $9.8 \%, 3.6 \%$ & $27.4 \%$ & $24.4 \%, 5.1 \%$ \\
\hline
\end{tabular}




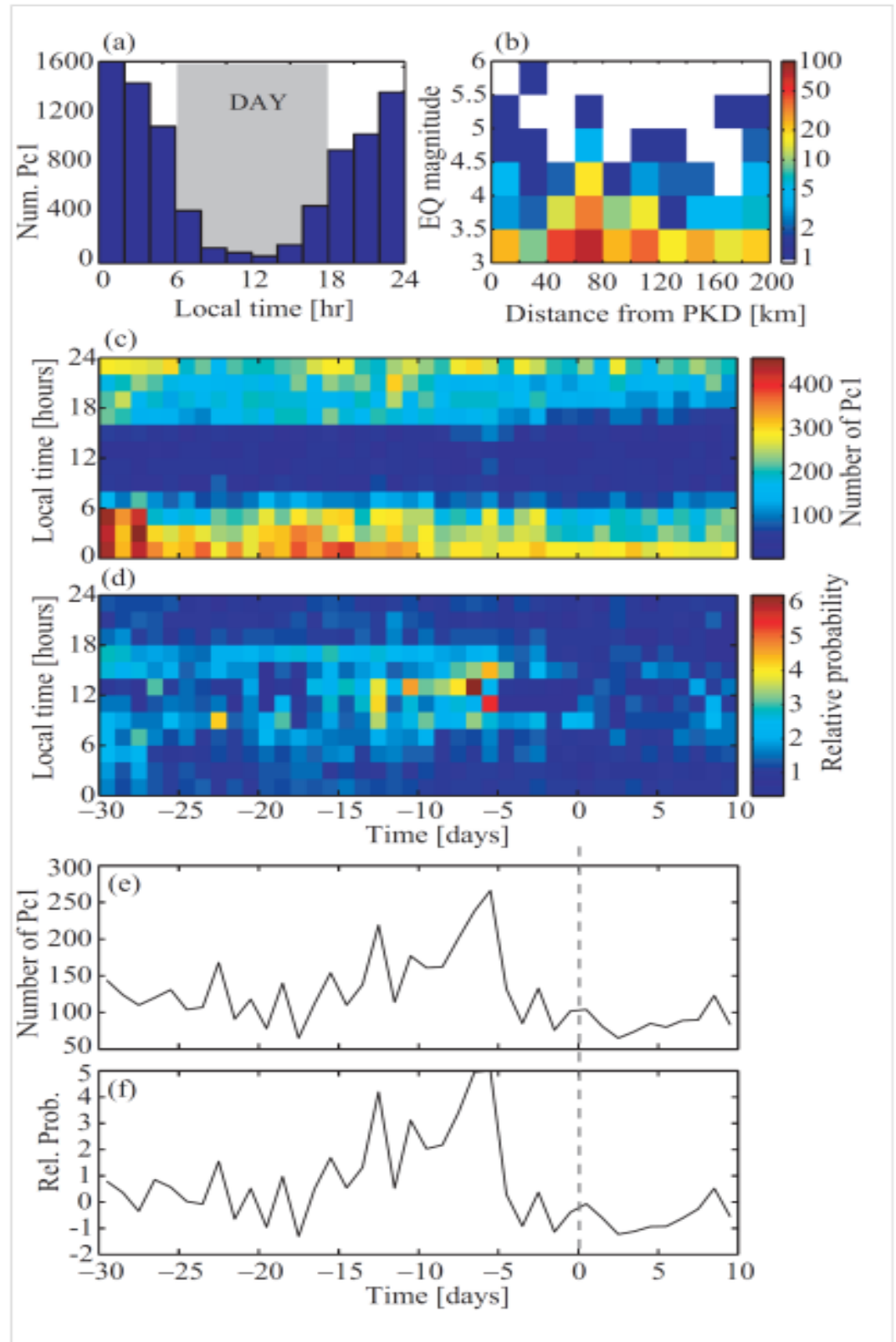

Figure 30: (a) Distribution of 8,193 Pc 1 pulsations as a function of local time (LT). (b) Distribution of 434 EQs during the period under consideration, as a function of EQ magnitude, and distance from our search-coil. (c) SPE analysis of Pc 1 events observed within -30 to +10 days of our 434 EQs, as a function of $\Delta T$ and LT. (d) Relative probability of observing Pc 1 pulsations in the same format as (c). (e) Number of Pc 1 pulsations observed on the day side, 08:00-16:00LT, corresponding to panel (c). (f) Same as (e), but corresponding to panel (d). After Bortnik et al., (2008) with permission from EGU. 


\section{VLF electromagnetic emissions}

This topic is a rather old one because the publication by Gokhberg et al., (1982) is one of the pioneering papers in the field of seismo-electromagnetics. There have been published numerous papers on this subject, and the early reports before 1990s (e.g.,Morgounov, 1985; Yoshino et al., 1985) were reviewed in a book by Gokhberg et al., (1995). Later reports on this subject were mainly presented in the monographs by Hayakawa and Fujinawa (Ed) (1994), Hayakawa (Ed) (1999), and Hayakawa and Molchanov (Ed) (2002). Below we will show some important contributions to this VLF study, including those by the groups led by Oike, Fujinawa, and Asada.

\subsection{One-site observation (Statistical study)}

Oike and Yamada (1994) looked at their 10-year statistics of electromagnetic noise in the frequency of VLF and LF. Their LF noise was monitored by a conventional radio receiver whose frequency is fixed at $163 \mathrm{kHz}$, and VLF noise was monitored by a ball antenna with a bandpass filter from $1 \mathrm{kHz}$ to $10 \mathrm{kHz}$. The variation in the number of LF and VLF electromagnetic noises at a particular station at Uji in Kyoto prefecture has been continuously observed since 1983.

Based on the 10-year data in LF and VLF, they performed the SPE analysis, and have found that an anomalous increase in LF noises has been observed not more than two days before the main shock of $70 \%$ of EQs with $\mathrm{M}$ greater than or equal to 6.0 whose epicentres are in the landor in the shallow sea regions in and around Japan. Similar results have been observed at VLF before nearby shallow EQs with M greater than 5.0. But, on the other hand, they discovered a clear correlation between VLF/LF noise and number of lightning strokes registered by HF monitoring system of Japan Meteorological Agency (JMA), and this point will be of essential importance in the later discussion on their nature and generation mechanism.

\section{2. VLF network observation}

The idea of network observation is not new, because Yoshino et al., (1985) had already attempted this kind of direction finding based on the triangulation method. Here we will cite the work by the group by 
Fujinawa.Matsumoto et al., (1996) and Fujinawa et al., (1999) established a direction-finding system for electromagnetic radiation pulses in the frequency range of $1-10 \mathrm{kHz}$ with the use of a network of 4 reception stations- Hasaki (HAS), Chikura (CKR), Kofu (KOF), and Nagaoka (NAG) (indicated by black squares)- located around Tokyo at distances of about 100-200 km as shown in Figure31 and equipped with antennas for 5 components of the signal ( 3 magnetic +2 electric components) as shown in Figure 32. The principle of this direction finding is different from the above by Yoshino et al., but is based on the time of arrival method. The followings were the results by Fujinawa et al., (1999).

Figure 33 illustrates an example of the number of VLF pulses including an interval around the date of a nearby strong EQ on March 6, 1996 (M=5.8). Their direction finding system allowed them to determine the distance, azimuth and height of the radiation source for each pulse. Due to the intensity threshold, the limiting distance was about $500 \mathrm{~km}$, and by a special requirement of pulse coincidence, local interferences were excluded. An example of VLF pulse signals on the resulting spatial distribution of radiation sources during 9 days in February and March 1997, when there was a seismic swarm east off Izu peninsula in Figure 34, was compared with the data from two lightning monitoring systems. Though the data amount was not large enough, Fujinawa et al., (1999) discovered two groups of electromagnetic radiation pulses:

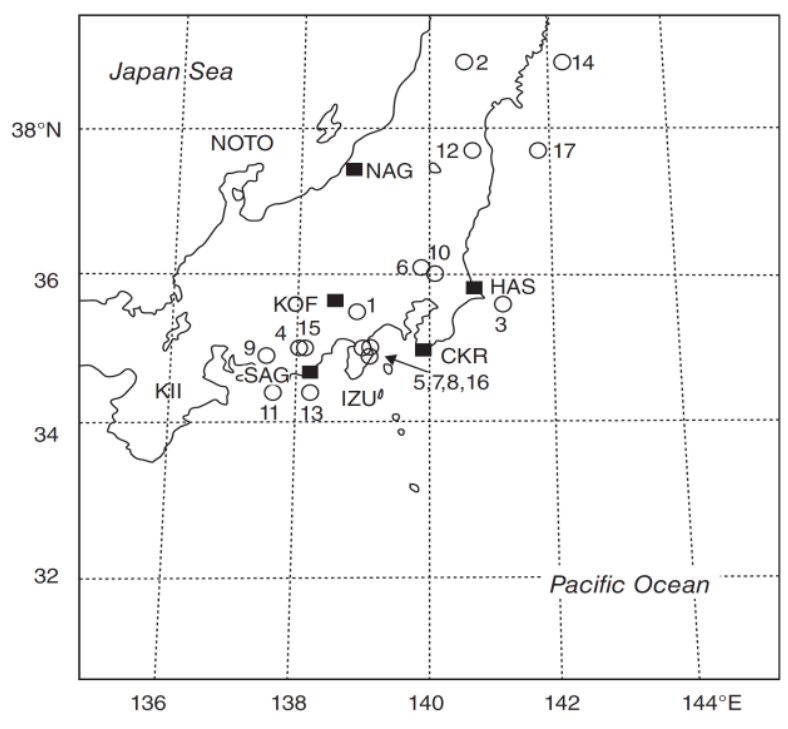

Figure 31: Locations of electromagnetic field observation sites in the central part of Japan $(\mathbf{\square})$.Circles $(\bigcirc)$ indicate the epicenters of EQs used in this study. Reproduced from Fujinawa et al., (1999) with permission from TERRAPUB. 


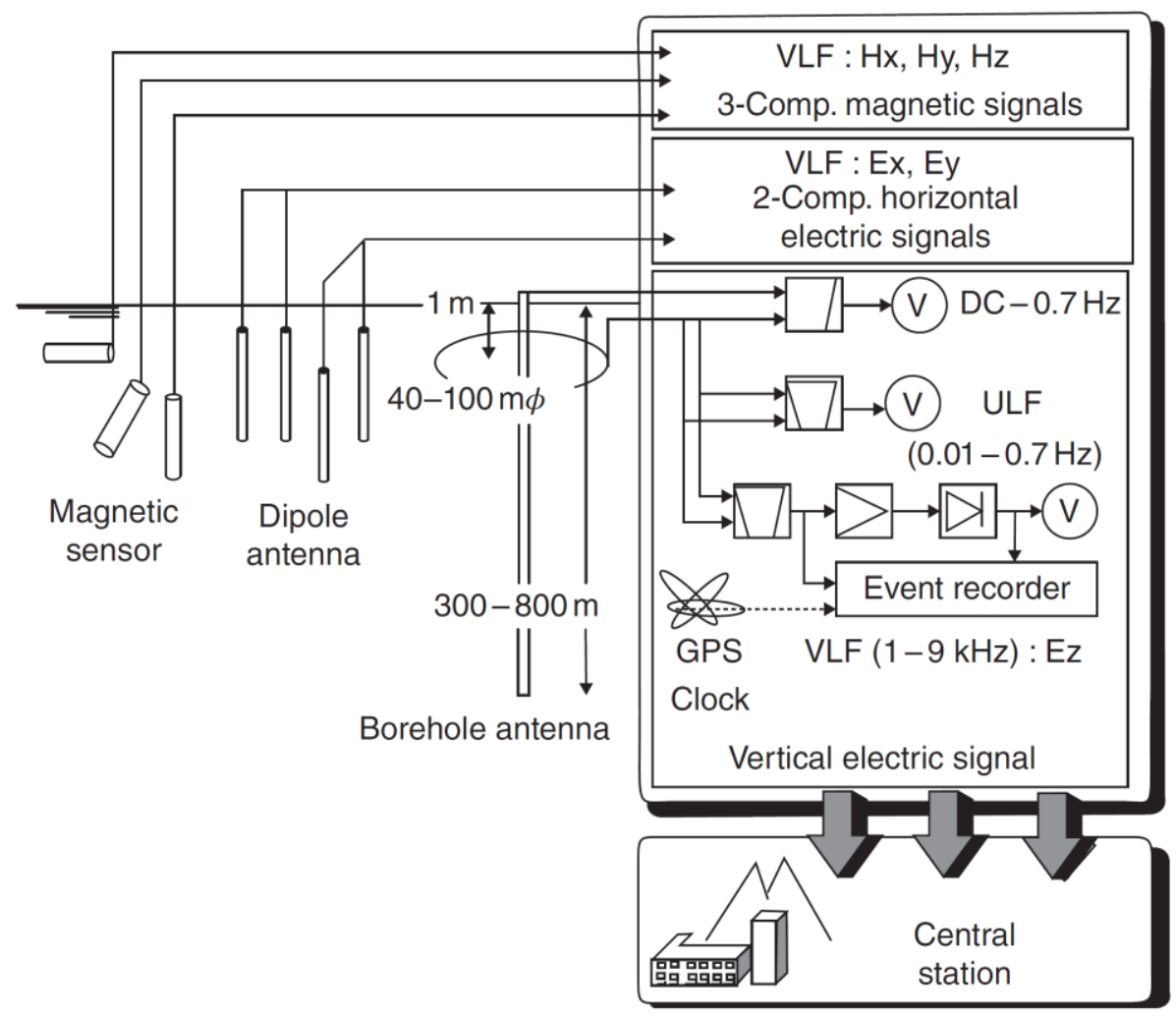

Figure 32: Schematic diagram of multiple-component measuring equipment used to locate the source point of electromagnetic pulses. Electric field was measured using a pair of horizontal dipoles that is $8 \mathrm{~m}$ long in length at distance of 20-50 m apart and using a vertical monopole that is $600-800 \mathrm{~m}$ long and is surrounded by a circle of wire (with a radius $10-20 \mathrm{~m}$ ) buried at the depth of approximately $1 \mathrm{~m}$. Magnetic field was measured by three mutually perpendicular induction-type magnetometers. Recorded data were transmitted to a central station once every night. After Fujinawa et al., (1999) with permission from TERRAPUB.

The first-group was correlated with lightning strokes, but the second was not correlated with cloudground strokes, as shown in Figure33. The latter was especially intensified about 1-2 days before the EQ and showed narrower distribution both in azimuth sector and in height. Those authors concluded:

(1) EQ-related pulses are generated in the troposphere above the sea.

(2) The amplitude of EQ-related pulses is on the order of lightning pulses, but the spatial distribution of their sources is different from that of both ground-cloud and cloud lightning strokes. 
(3) The results based on their limited data amount support earlier statistics by Oike and Yamada (1994) on the point of temporal evolution (lead time of noise from EQ date), but are very different from theirs on the spatial distribution.

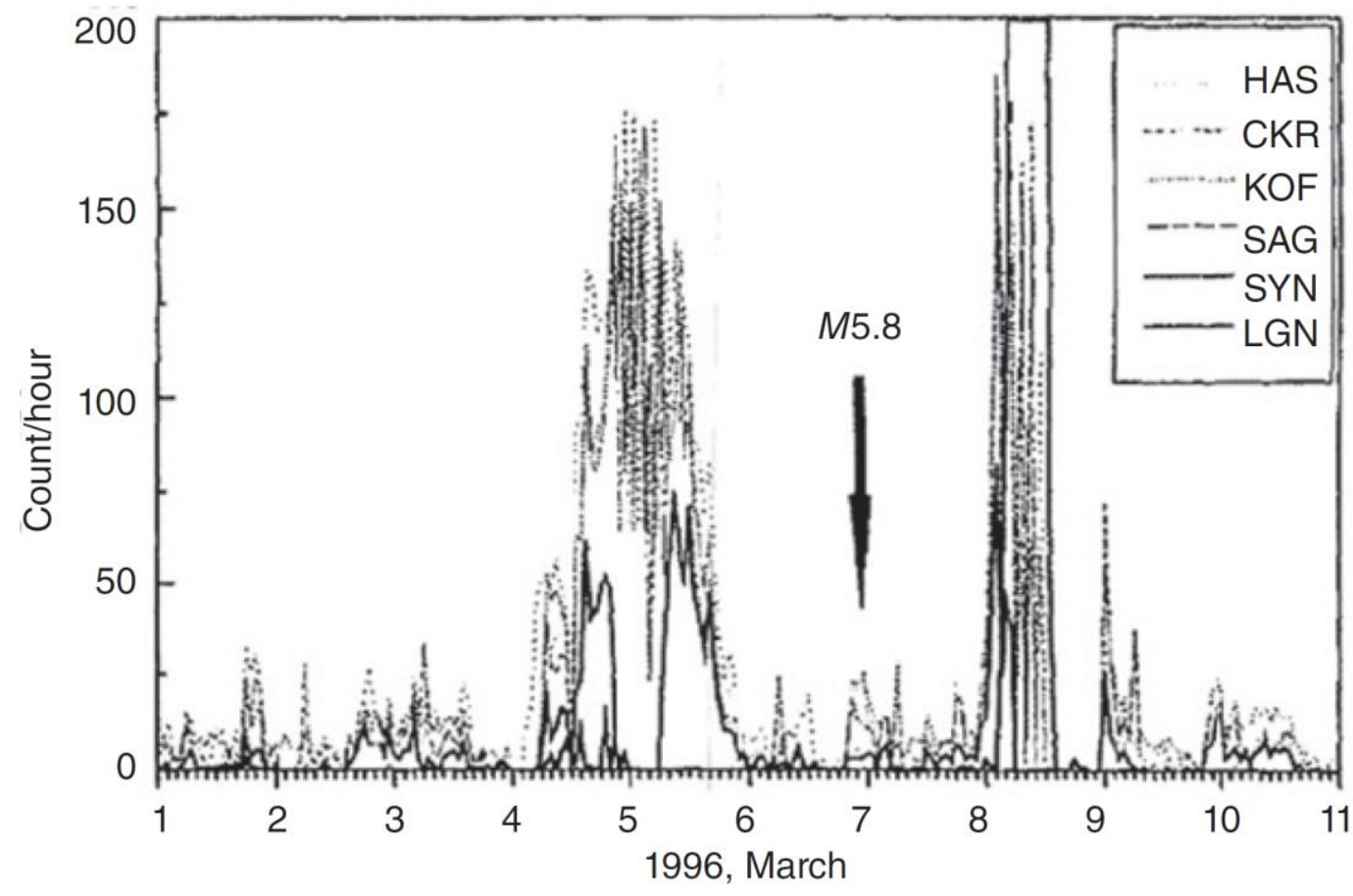

Figure 33: The number of VLF pulses per hour around the time of a major shallow EQ of M=5.8 in the network at four sites. SYN means event numbers identified to be the same signal through the time window criterion. Signals not included in the synchronized events are thought to be local noises or local events except at two time periods of most prominent VLF emission on early March 5 and 8 when the data storage facility did not work due to too many events. Also shown is the number of lightning discharges to ground (return stroke) observed by the lightning monitoring network covering Honshu in Japan. After Fujinawa et al., (1999) with permission from TERRAPUB. 


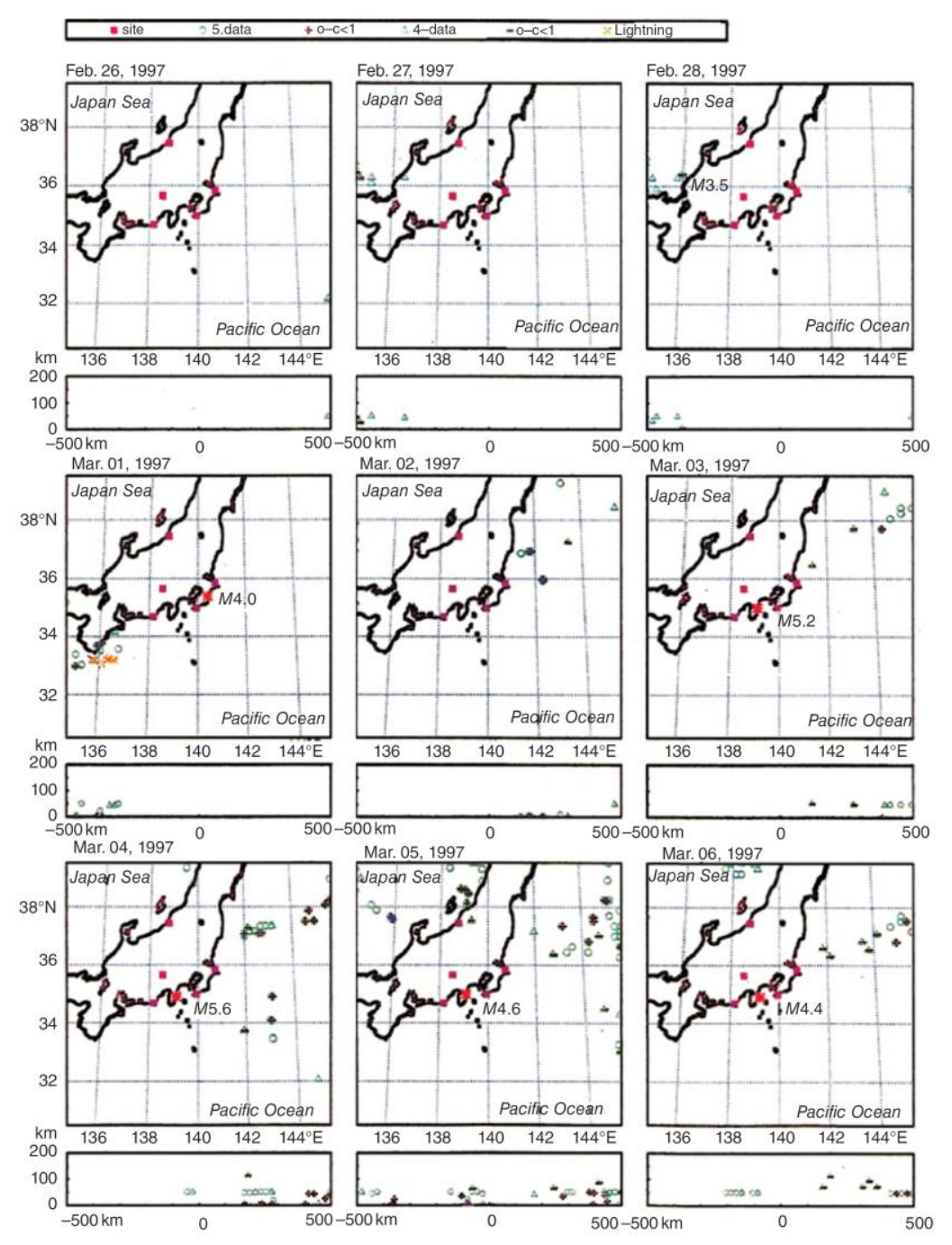

Figure 34: Source point projections for 9 days around the day of seismic swarm east off Izu peninsula with major EQs with Ms of 5.7 and 5.8 on March 3-4, 1997. In this case, there were neither electromagnetic pulses around the epicentral region nor any indication of an increase in pulse number height. After Fujinawa et al., (1999) with permission from TERRAPUB.

\section{3. Distinction between meteorological lightning and seismogenic VLF emissions}

Fujinawa et al., (1999) made an attempt to distinguish between the conventional lightning due to meteorological effects and seismogenic VLF emissions. They concluded that those VLF emissions are impulsive, so that the main problem remains on how to distinguish between the two with the comparison of lightning distribution by the HF (high frequency) lightning monitoring system. 
Here we have to cite an important paper by Asada et al., (2001), who have made an attempt to delineate VLF seismogenic emissions with the use of direction finding triangulation with three stations equipped with terrestrial (nor borehole) antennas in Japan. They have presented several examples that demonstrate positively the existence of a class of VLF signals having a peak frequency of occurrence 1-4 days prior to EQs with M 4-6.The VLF sources by Asada et al., (2001) are located in the land, being consistent with Oike and Yamada (1994) but inconsistent with Fujinawa's conclusion. The direction finding method by Asada et al., is expected to be of potential use in distinguishing between the seismogenic and meteorological emissions.

Similar borehole observations of electromagnetic noise have been carried out by some other groups (Singh et al., 2003; Tsutsui, 2012). In the study by Singh et al., (2003) they measured vertical electric field components of VLF electromagnetic emissions at a frequency of $3 \mathrm{kHz}$, employing both borehole (depth, 100m) and terrestrial antennas. Then, they found three different kinds of abnormal electric field changes: (i) noise bursts observed only by a borehole antenna, (ii) noise bursts observed only by a terrestrial antenna, and (iii) noise bursts observed by both antennas. Finally, they concluded that the first kind of noise is likely to be positively correlated with major seismic activities in the region of their interest. Tsutsui (2012) further used borehole antennas with the similar length to the earlier case, he tried to distinguish between seismogenic emissions and other noise (like lightning discharges), but were not so successful.

The use of borehole antennas in the frequency ranges of VLF and ELF cited above, were initially intended in order to detect directly electromagnetic emissions originated in the lithosphere, but the reception characteristics of such borehole antennas have not been investigated extensively, so that we are not sure what we measure by receiving electromagnetic waves by borehole antennas (either reception of direct lithospheric emissions or the reception of seismogenic waves generated near the epicentre and entering and propagating in the Earth-ionosphere waveguide). Though the history of VLF emissions is the longest as compared with other items, those characteristics are poorly understood, and left unanswered nowadays. 


\section{EQ effect on VLF whistlers}

Whistlers are typical ELF/VLF signals mainly observed at high and medium-latitudes (e.g., Helliwell, 1965), but even at low latitudes (geomagnetic latitude 20-30) (Hayakawa, 1995). This point is essentially different from Pc 1 pulsations whose path latitudes are only high latitudes. Hayakawa and Tanaka (1978) concluded based on different observational evidence, that whistlers at these low latitudes as in Japan are trapped in field-aligned ducts as in the case of high and middle latitude whistlers (Park, 1982). The time delay of whistlers is defined as the dispersion value (D), which is given by $D=\mathrm{tf}^{1 / 2}$, where $t$ is the time delay and $\mathrm{f}$ is the wave frequency. Sometimes, a whistler bounces back and forth between both hemispheres, and the dispersion values of such whistler echoes will be $\mathrm{D}, 3 \mathrm{D}, 5 \mathrm{D}$, etc., when the lightning is in the hemisphere opposite to that of the observer. When both the source and observer are in the same hemisphere, we observe whistlers with 2D, 4D etc. The whistler with the fundamental $\mathrm{D}$ is called a "short whistler" and that with 2D, a "long whistler". The following result is based on the paper by Hayakawa et al., (1993).

\subsection{Whistler data used}

At the Sugadaira Observatory [geographical coordinates, $36^{\circ} 31^{\prime} \mathrm{N}, 138^{\circ} 19^{\prime} \mathrm{E}$; geomagnetic latitude, $26^{\circ} \mathrm{N}$; L (McIlwain L-parameter, L is defined by the equatorial radius of a magnetic field line normalized by the Earth's radius) $=1.33$ ] of UEC, wideband (up to 10kHz) VLF recordings were carried out according to the international whistler monitoring schedule (for 2 min every hour) over a 9-year time span (1970-1978). The VLF receiver system consisted of orthogonal loop aerials, a two-channel standard wideband receiver, and a data recorder that was connected with a $20 \mathrm{~dB}$ preamplifier. The data from Moshiri Observatory (geographic coordinates, $44^{\circ} 26^{\prime} \mathrm{N}, 142^{\circ} 47^{\prime} \mathrm{E}$; geomagnetic latitude, $35^{\circ} \mathrm{N}$; $\mathrm{L}=1.59$ ) of Nagoya University, were used as supplements. Observations at Sugadaira ceased in 1979, and instead, we made use of data observed at Sakushima (geographic coordinates, $34^{\circ} 43^{\prime} \mathrm{N}, 138^{\circ} 02^{\prime} \mathrm{E}$; geomagnetic latitude, $\left.24^{\circ} \mathrm{N} ; \mathrm{L}=1.28\right)$ of Nagoya University for 3 years (1980-1982). 


\subsection{Definition of anomalous whistlers}

The values of $\mathrm{D}>70 \mathrm{~s}^{1 / 2}$ can be regarded as being unusual or anomalous at our latitude station. Echo trains of a short whistler and a long whistler are rather frequent at middle and high latitudes (geomagnetic latitude $>45^{\circ}$ ), so the echo index (defined as the ratio of the number of whistlers whose echoes were observed and the total number of whistlers) amounts to $\approx 0.5$ (Helliwell, 1965), but these were extremely rare at our lower latitude stations. The probability of occurrence of echo trains is still not relatively high, of the order of less than $0.1 \%$ at Sakushima (Hayakawa and Tanaka, 1978), and whistlers with D>70s ${ }^{1 / 2}$ were much less than $0.1 \%$ of the total whistlers at Sugadaira. Hayakawa and Otsu (1973) have elucidated, on the basis of multi-station network observation, that whistlers exiting the ionosphere at a latitude lower than that of an observing station (corresponding to the dispersion smaller than the typical D) tend to propagate mainly towards higher latitudes, which is a characteristic peculiar to low latitudes. Also, we have known based on long-term experience that whistlers with larger dispersion values which have penetrated the ionosphere at middle and high latitude have rarely reached our low-latitude station via the Earth-ionosphere waveguide. Furthermore, the direction finding measurements by Tsuruda and Hayashi (1975); Okada et al., (1977) ; Ohta et al., (1984) and Hayakawa et al., (1990) have provided evidence that the ionospheric exit region of whistlers observed at low latitudes (such as at Sugadaira and Sakushima) is usually close to the station.

Case studies of several examples of the EQ effect on low latitude whistlers were presented in the paper by Hayakawa et al., (1993), but below we present only their statistical results.

\subsection{Statistical Results}

Figure 35 illustrates the geographical setting of the whistler observing stations (Sugadaira, Sakushima, and Moshiri) and their conjugate points. The full line in Figure 35 illustrates the projection on the ground of the magnetic field line connecting Sugadaira and its conjugate point. The occurrence of whistlers is controlled by two factors: (i) source activity and (ii) propagation condition in the ionosphere and magnetosphere, but the relative importance of these two factors seems to depend on latitude (Ohta and Hayakawa, 1990). 


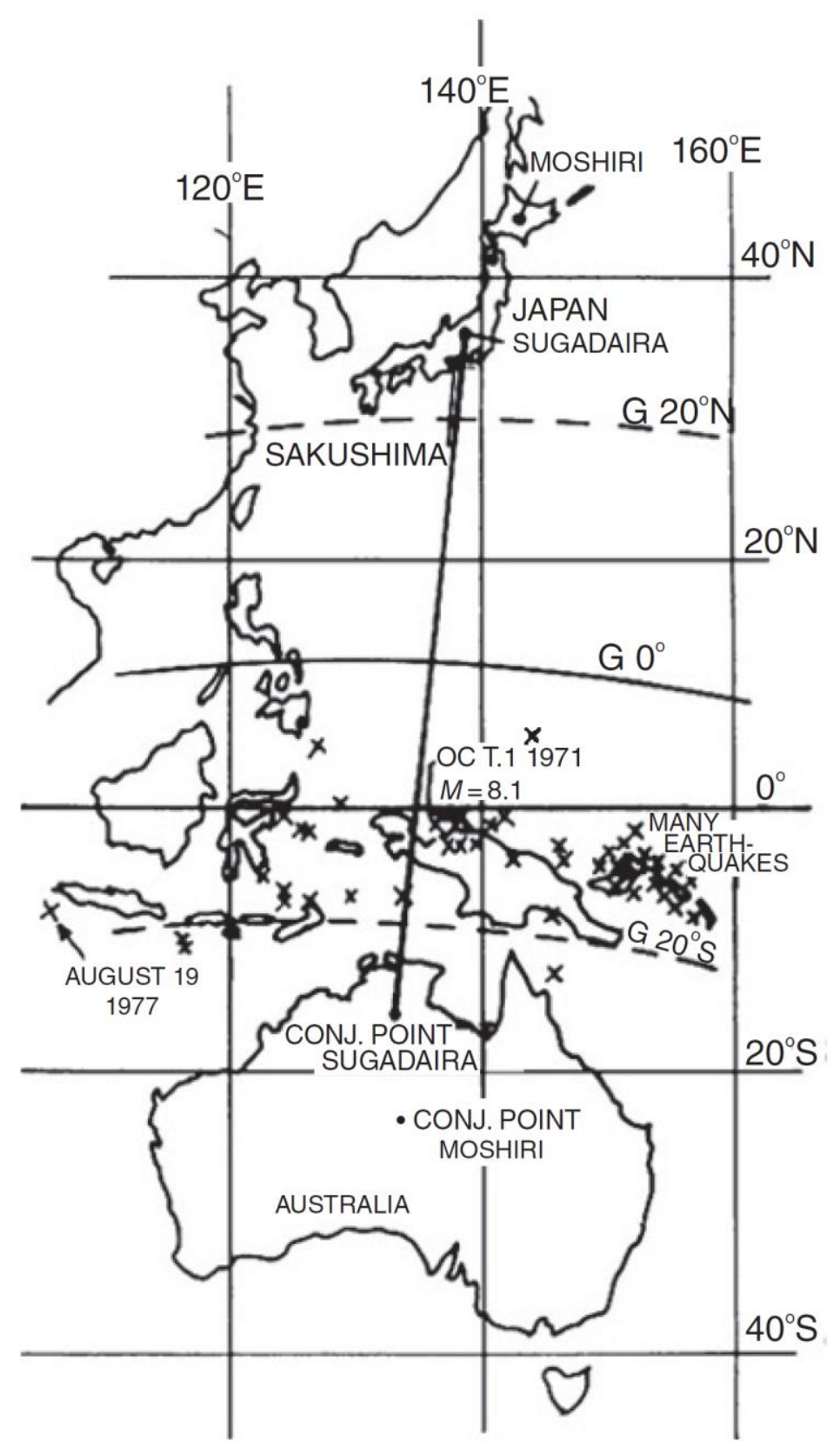

Figure 35: Locations of Sugadaira, Sakushima, and Moshiri whistler observatories and their conjugate points and epicentres of EQs $(M \geq 6)(\times)$ in the longitude sector of 100-160 ${ }^{\circ}$ E during 1970-1978. The full line indicates the ground projection of the field line connecting Sugadaira and its conjugate point. Reproduced from Hayakawa et al., (1993) with permission from Elsevier. 
A strong seasonal variation in whistler occurrence, especially at low latitudes [maximum in winter (austral summer) and minimum in summer (austral winter)], can be explained in terms of the corresponding seasonal variation of lightning activity in the southern hemisphere. However, this does not mean that the source activity is necessarily the dominant factor. Ohta and Hayakawa (1990) have suggested that the source activity is just a necessary condition for the occurrence of whistlers and the propagation condition (such as the absorption in the ionosphere and duct formation) is much more influential than the source effect, especially at low latitudes. Since the field lines supporting the whistler propagation terminate very close to each observing station, as evidenced in direction finding studies, etc., we selected the EQ events in a tentatively chosen local sector of the geographic longitude of $100-160^{\circ} \mathrm{E}$, but we later found that this selection did not influence our conclusion which will be presented later. In Figure 35, we also plotted, by crosses, the location of all the EQs satisfying the earlier longitudinal conditions and with M exceeding 6 during our analysis period of 1970-1978. Their locations are localized relative to the range in geographical latitudes of $10^{\circ} \mathrm{N}$ to $15^{\circ} \mathrm{S}$, which are closely associated with the area of the Philippine Sea plate.

As mentioned previously, the whistler occurrence exhibits conspicuous seasonal variations (Hayakawa et al., 1971), and thus the appearance of any kind of whistlers (either short whistlers with D or anomalous ones) in June-August is quite a rare phenomenon at our station of Sugadaira.

Additionally, it is reasonable to regard the whistlers with $\mathrm{D}>70 \mathrm{~s}^{1 / 2}$ during this interval of June-August as "obviously anomalous events". The broken line in Figure 36 indicates the number of EQs in each month for 1975, and the full line indicates the monthly variation of the number of days on which anomalous whistlers were detected at Sugadaira. We have counted all EQs with M>6 and the number of days with anomalous whistlers. This figure suggests a close correlation between the occurrence of anomalous whistlers and EQs in the Japanese longitude because the curve of whistler data in full line follows nicely in parallel with that of the local EQ activity in the broken line, except in October when there was enhanced EQ activity. Later examination of the local noise interference indicated that it was unexpectedly enhanced in October, which might be the major reason for no correlation in Figure 36 . It seems from Figure 36, that the summer period is especially suited for determining a relationship between seismic influence (effect) and whistler propagation. 


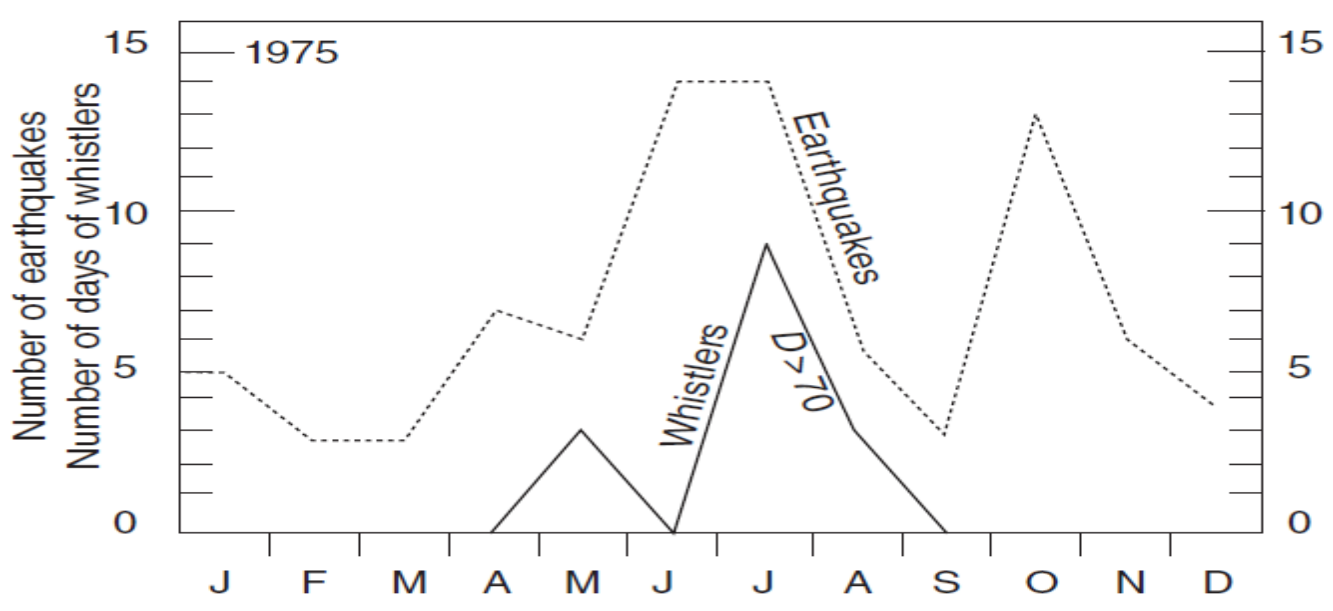

Figure 36: Relation between the monthly number of EQs with magnitude $M \geqq 6$ and the number of days of appearance of anomalous whistlers with dispersion D $>70$ at Sugadaira in 1975. A solid line indicates the number of days with anomalous whistlers. Reproduced from Hayakawa et al., (1993) with permission from Elsevier.
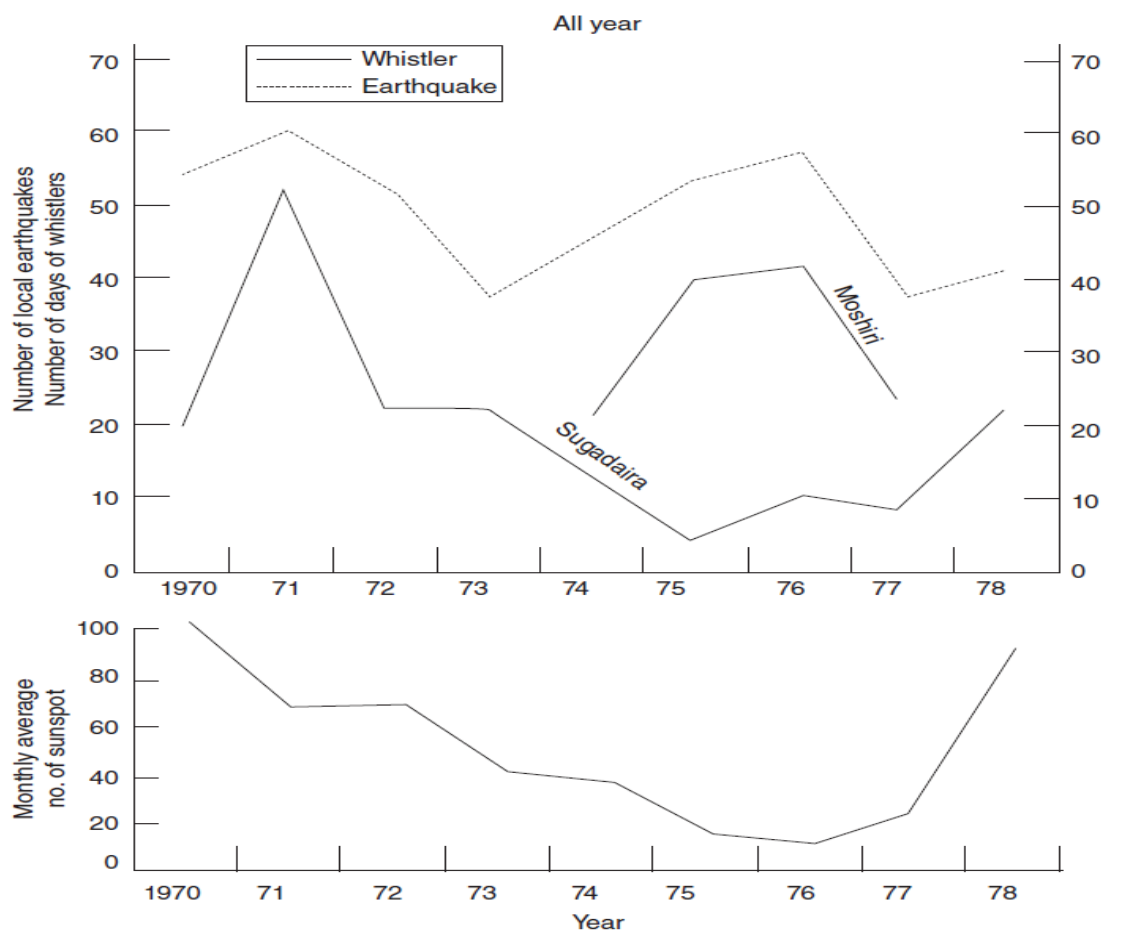

Figure 37: Statistical results of comparison between the annual number of EQs with $M$ greater than 6 and the number of days with anomalous whistlers observed at Sugadaira during the period of 1970-1978. The bottom panel indicates the monthly average of sunspot numbers. Reproduced from Hayakawa et al., (1993) with permission from Elsevier. 
Figure 37 illustrates the corresponding statistical results at Sugadaira during the whole time span of 1970-1978. In drawing this figure, we counted the number of days with anomalous whistlers, and the EQs in the Japanese longitude sector for each year. For the half period until 1973 (Figure 37), we found very clearly a close correlation between the number of days with anomalous whistlers at Sugadaira and corresponding seismic activity in the Japanese longitude (the correlation coefficient being $\mathrm{r}=0.63$ ). Conversely, the correlation between the two quantities is rather poor for the latter half period in Figure 37; however, as already mentioned, the condition of VLF measurement was not good at Sugadaira in 1975 and 1976, and the number of days with anomalous whistlers for these 2 years at Sugadaira is likely to be shifted to a higher level. The extent of shift is quite uncertain, but we can recover some useful information from the corresponding data for Moshiri in the same figure. The data at Moshiri were plotted in order to supplement the Sakushima data in the period of 1974-1977 only, and the correlation coefficient $r=0.84$. In conclusion, we were able to establish that whistlers and EQs are likely to be closely related to each other during the entire period of analysis. At the bottom of Figure 37, the annual variation in solar sunspot number $\mathrm{Z}$ is plotted, because it is known that the occurrence rate of short (common) whistlers is anti-correlated with the sunspot number at our low latitudes (Ohta and Hayakawa, 1990). The comparison with the variation of sunspot numbers shows no relationship between anomalous whistlers and sunspot activity.

No further studies have been unfortunately performed since the paper by Hayakawa et al., (1993), except one by Liu et al., (2013), so it is highly required to carry out this kind of studies in future on further correlation studies between whistlers at different latitudes and EQs.

\section{Lightning activity}

This topic seems to be closely related to the subject of seismogenic VLF electromagnetic emissions in Section 6. Lightning activity as a signature of discharges in the atmosphere, has been investigated in Taiwan using the 12-year period of 1993-2004 (Liu et al., 2015). Unfortunately there is written no detailed information on which kind of lightning detector was used for lightning activity (probably by $\mathrm{HF}$ lightning detector), but we present a recent result by Liu et al., (2015). Their study is considered to be a further extension of the earlier work by Oike and Yamada (1994). 


\subsection{Case study}

The bottom panel of Figure 38 illustrates an example of temporal evolution of lightning activity (number of lightning discharges) that indicates that lightning occurrence along the Chelungpu fault (as marked by a rectangle in the top panels) was significantly enhanced 20-30 days and 2-7 days prior to the 2 September 1999 (0147 LT) M7.6 Chi-chi EQ. The lead time of 2-7 days is likely to be consistent with the result by Oike and Yamada (1994).

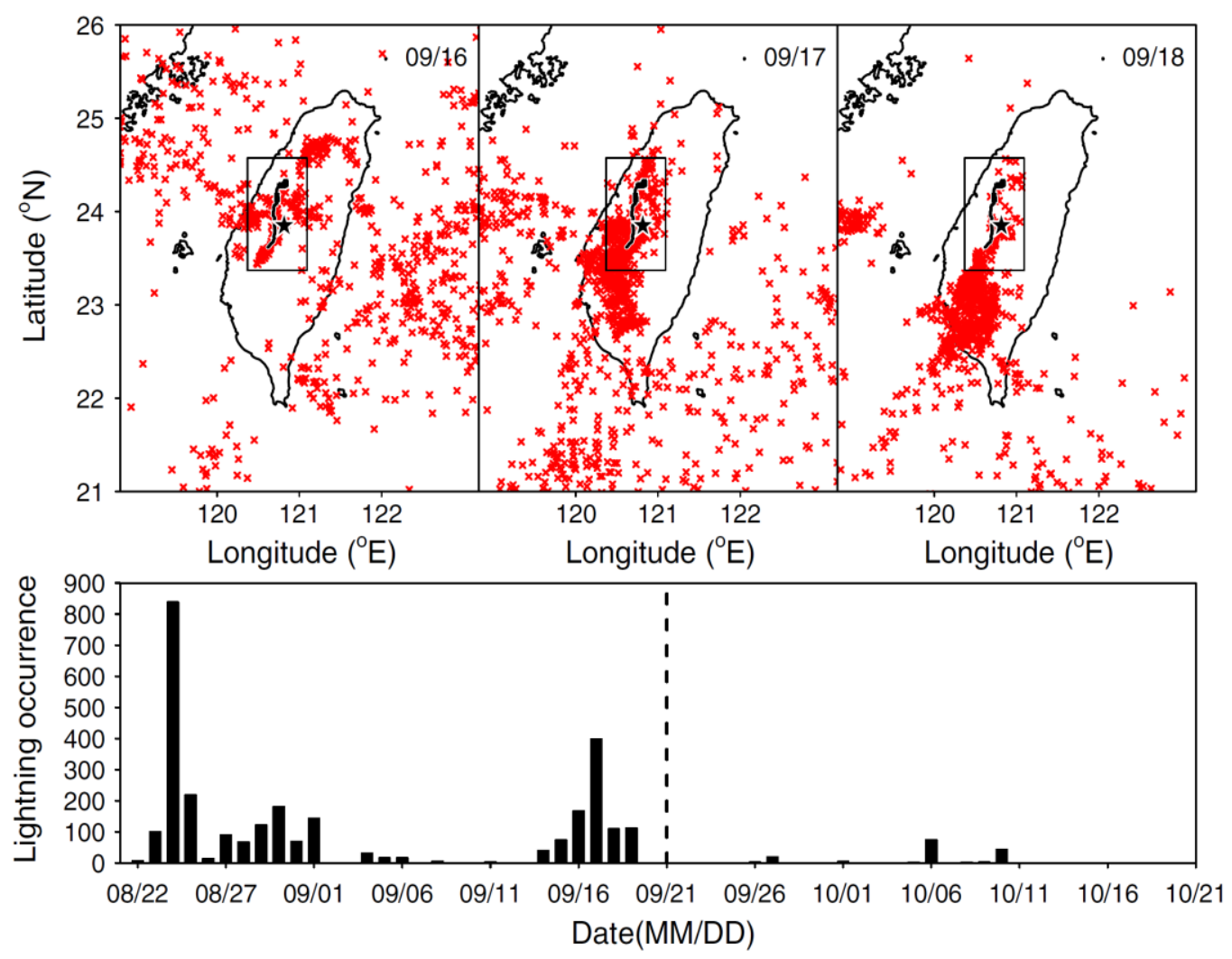

Figure 38: Lightning activities during the 21 September 1999 M7.6 Chi-chi EQ. Top panel on the spatial distribution of lightning on 16, 17 and 18 September are shown in the upper panels. Lower panel is the lightning occurrence/ counts within $0.25^{\circ}$ latitude or longitude around the Chelungpu fault denoted by the rectangular box 130 days before and after the Chi-Chi EQ. In fact, $90 \%$ of the lightning occurred before the Chi-chi EQ in the 61 days of this study period. After Liu et al., (2015) with permission from Springer. 


\subsection{Statistical study}

Figure 39 illustrates the location of the $308 \mathrm{M} \geq 5.0$ EQs that occurred in the Taiwan region during 19932004. The circle and cross symbols denote the 78 land and 230 sea EQs, respectively. Figure 40(a) refers to all $9 \mathrm{M} \geq 6.0$ land EQ during the observation period as an example. It can be seen that the proportion increases monotonically from day 1 to 7, becoming saturated thereafter, and yields maxima on days 17-19. Figure 40(b) illustrates the proportions of various magnitudes and cumulative days for both sea and land EQs. The proportions of sea EQs are less than 0.5, while those of land EQs increase with their magnitudes. It can be seen that a larger land EQ yields a greater proportion within a shorter cumulative day, and more than $70 \%$ of $M \leq 5.8$ land EQs are associated with the anomaly for longer than seven cumulative days.

To find whether such an enhancement is also likely to appear before other EQs, a statistical analysis is implemented to examine lightning occurrence associated with $395 \mathrm{M} \geq 5.0 \mathrm{EQs}$, occurring over $308 \mathrm{EQ}$ days in Taiwan during the 12-year period of 1993-2004 as in Figure 40 when several $\mathrm{M} \geq 5$ EQs occur on the same day, only the longer or largest one is adopted. It seems that their results are the confirmation of Oike and Yamada (1994)'s result, but there exist an important question whether they are really seismogenic or not.

As the conclusion of statistical results, it is found that lightning activities tend to appear around the forthcoming EQ epicentre and are significantly enhanced, especially 17-19 days before the $M \geq 6.0$ shallow (depth $\leq 20 \mathrm{~km}$ ) land EQs. Moreover, the size around the EQ epicenter with statistical significance of lightning activity enhancement is proportional to the EQ magnitude.

Similar statistical studies will be highly required before a definite conclusion on the correlation of lightning activity with EQs is reached. 


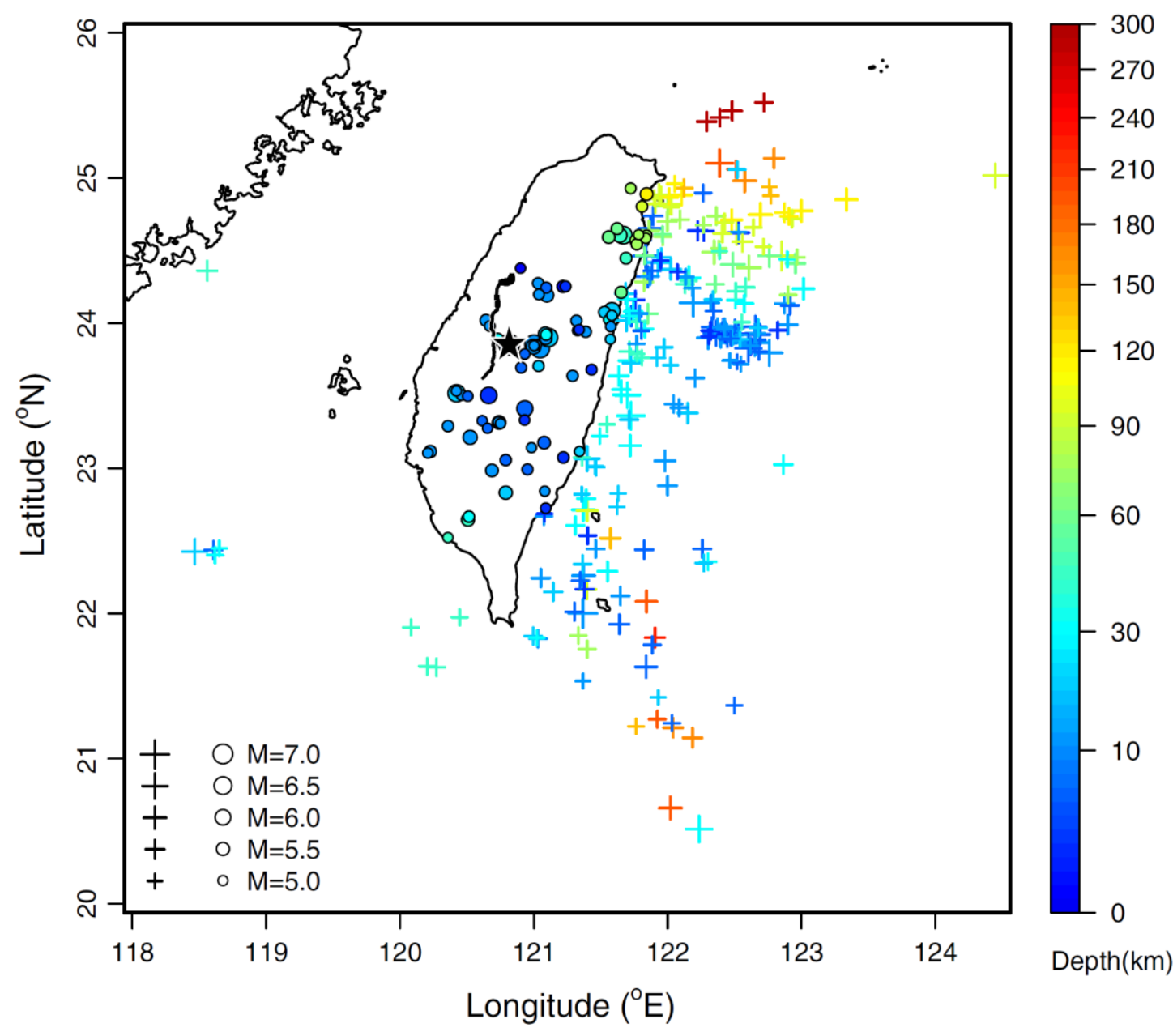

Figure 39: The locations of the $308 \mathrm{ML} \geqq 5.0 \mathrm{EQs}$ that occurred in the Taiwan region during 1993-2004. The circle and cross symbols denote the 78 land and 230 sea EQs, respectively. The black star and curve denote the 21 September 1999 M7.6 Chi-chi EQ (23.86N, 120.82 E) and the Chelungpu fault, respectively. After Liu et al., (2015). 
(a)
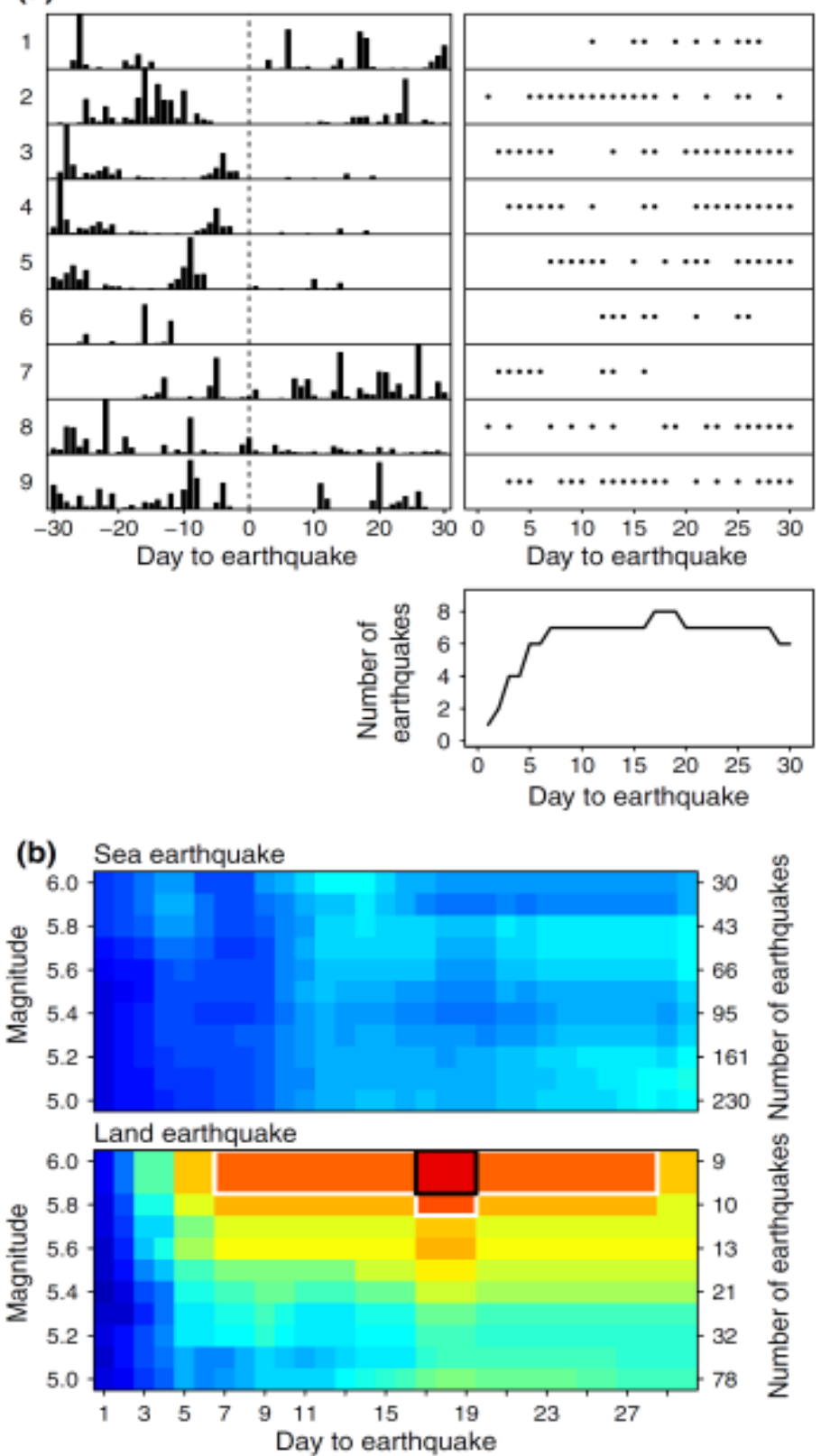

$\begin{array}{lllllllllll}0.0 & 0.1 & 0.2 & 0.3 & 0.4 & 0.5 & 0.6 & 0.7 & 0.8 & 0.9 & 1.0\end{array}$

Proportion

Figure 40 (a): The proportions and associated $\mathrm{P}$ value of various EQ magnitudes and cumulative days. A left panel is lightning activities during each $\mathrm{M} \geqq 6.0$ land $\mathrm{EQ}$; right top panel pre-EQ lightning anomaly within various cumulative days of each event; lower right panel reveals the summation of the EQ number with anomaly for a certain cumulative day. (b) The proportions of the sea EQs (upper) and land EQs (lower) with anomaly in various cumulative days and magnitudes. The EQ numbers with different magnitudes are given on the right-hand side. The black and white rectangular areas denote $\mathrm{P}$ values being less than 0.05 and 0.10 , respectively. The $\mathrm{P}$ value in the black rectangle is 0.02. After Liu et al., (2015). 


\section{Possible physical modeling}

Here again we repeat the items of our interest in the ULF/ELF/VLF ranges: (i) ULF/ELF radiation, (ii) Anomalies in SR, (iii) ULF magnetic field depression, (iv) EQ effect on Pc1 pulsations, (v) VLF/ELF electromagnetic emissions, (vi) EQ effect on VLF whistlers, and (vii) Lightning. Historically speaking, the former four phenomena are considered to be rather new, while the latter three phenomena are relatively old topics. Correspondingly the mechanisms of the former four phenomena are poorly understood. Then, even though the latter topics have been studied in the early stage of 1980s, the generation mechanisms of those effects are still left unanswered.

Then, it is natural for us to categorize the above phenomena roughly into the two types from the physical point of view. One seems to be related the ionospheric anomaly ((ii), (iii), (iv), and (vi)) and the other is related with discharge type in the atmosphere ((i), (v), and (vii)).

Here we start with the phenomena seemingly to be associated with ionospheric perturbation. In the field of seismo-electromagnetics, the presence of seismo-ionospheric perturbation has been studied for the last three decades and is considered to be well established observationally. Of course the mechanism on how and why those ionospheric perturbations are generated is poorly understood at the moment though there have already been proposed, even in early 2000s, a few hypotheses of LAIC. Further more detailed discussions on these hypotheses are being carried out in Hayakawa et al., (2004); Pulinets and Boyarchuk (2004); Molchanov and Hayakawa (2008); Sorokin et al., (2015); Hayakawa et al., (2018) and Ouzounov et al., (Eds) (2018).

\subsection{Seismo-ionospheric perturbations}

Emergence of lower ionospheric perturbations prior to a large EQ has already been evidenced by means of subionospheric VLF/LF propagation anomalies (Hayakawa et al., 1996a; Molchanov and Hayakawa, 1998; Rozhnoi et al., 2004; Biagi et al., 2004; Chakrabarti (Ed), 2010; Hayakawa, 2011; Rozhnoi et al., 2013; Hayakawa et al., 2018). Because these subionospheric VLF/LF signals are known to be reflected from the lowest ionosphere ( $\mathrm{D}$ region at day and lower $\mathrm{E}$ layer at night), it is already believed that the lowest ionosphere is perturbed a few days to about a week before large EQs. Furthermore, the upper F region of the ionosphere is also known to be perturbed before the major EQs with the help of 
observational data on ionosondes, GPS TEC (total electron contents), satellite observations, etc. (Pulinets and Boyarchuk, 2004; Liu, 1999; Sorokin et al., 2015; Devi et al., 2010; Liu et al., 2018; Parrot and Li, 2018; Ouzounov et al. (Eds), 2018).

A lot of evidence has been accumulated on the presence of perturbations in the ionosphere as presented in this paper. The elucidation of the generation mechanism of those ionospheric perturbations (or LAIC) is the final goal of our seismo-electromagnetic studies, and this interdisciplinary topic is extremely challenging.

Hayakawa et al., (2004) have already proposed a few possible hypotheses on the LAIC mechanism: (i) chemical channel, (ii) atmospheric oscillation channel, and (iii) electromagnetic channel. Figure 41 illustrates the schematic diagram of these three coupling channels. As for the first channel, radon emanation induces the perturbation in the conductivity of the atmosphere, a change in the atmospheric electric field that leads to the ionospheric modification through the atmospheric electric field (Pulinets and Boyarchuk, 2004; Sorokin et al., 2006; Rycroft et al., 2012).

Integration of informations in different regions (Lithosphere-atmosphere-ionosphere (LAI) coupling)

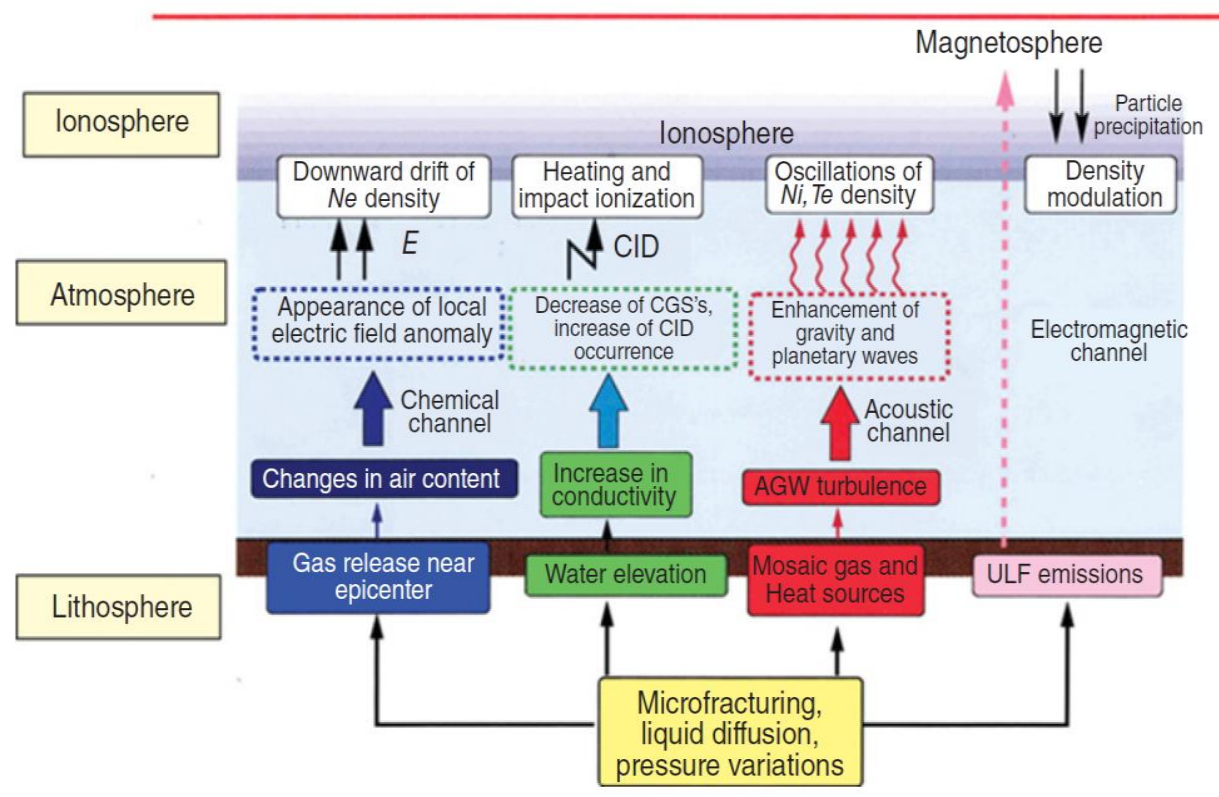

Figure 41: Schematic illustration of the lithosphere- atmosphere- ionosphere (LAI) coupling (i) chemical (+electric field) channel, (ii) atmospheric oscillation channel, and (iii) electromagnetic channel. Reproduced from Hayakawa (2009) with permission from Transworld Research Network (Trivandrum, India). 
The second channel is based on the key role of atmospheric oscillations (acoustic wave (AW) or atmospheric gravity wave (AGW)) in the LAIC, and perturbations in the Earth's surface (such as temperature, pressure) in a seism active region excite the atmospheric oscillations travelling up to the ionosphere and inducing ionospheric density perturbations (Molchanov et al., 2001; Miyaki et al., 2002; Hayakawa et al., 2011b). The last mechanism, (iii) electromagnetic channel, is that radio emissions (in any frequency range) generated in the lithosphere propagate up to the ionosphere and modify the ionosphere through heating and/or ionization. But this mechanism is found to be insufficient because of the weak intensity of lithospheric radio emissions (Molchanov et al., 1993), and so the first and second mechanisms are likely plausible candidates for this coupling at the moment. Pulinets and Boyarchuk (2004) and Pulinets and Ouzounov (2011) insisted on the first chemical channel as the most promising candidate for the ionospheric perturbations associated with EQs. That is, the emanation of radon was suggested as the main player of seismo-ionospheric perturbation, but there seems to be very little experimental (observational) evidence in support of their hypothesis, even though we know that there have been reports on the radon emanation itself (e.g., Yasuoka, 2012) as a precursor to an EQ. This chemical channel has been seriously criticized by Denisenko (2015) and Prokhorov and Zolotov (2017) because of its low efficiency. The most important signature associated with this first channel seems to be the generation of an electric field in the ionosphere in possible relationship with EQs. We will give a brief explanation here. Chmyrev et al., (1986) observed, using the Intercosmos-Bulgaria 1300 satellite, the anomalies in ULF $(0.1-8 \mathrm{~Hz})$ electromagnetic and quasi-static electric fields above the EQ epicentral zone and its conjugate region and also prior to an EQ. Such observations of seismogenic electric fields and associated phenomena within the ionosphere have been summarized by Pulinets and Boyarchuk (2004), Sorokin and Hayakawa (2013) and Sorokin et al., (2015). Unlike the generation of electric fields in the ionosphere, there have been no such significant changes in the electric field near the Earth's surface. Both of these phenomena of conspicuous electric fields in the ionosphere and no noticeable changes in electric field near the Earth's surface prior to an EQ would be an appropriate special subject to be discussed for the first channel.

Compared with the first chemical (+electric field) channel, a lot of experimental evidence has been accumulated in favour of the second channel (due to atmospheric oscillations) mainly by using subionospheric VLF data. Hayakawa et al., (2011b) have summarized different kinds of signatures of atmospheric oscillation effects in support of the second channel, such as the finding of many examples of 
enhancement of fluctuation in subionospheric VLF/LF data in the frequency range of AW and AGW before an EQ. In addition to much indirect evidence, we have provided here the first direct evidence of AGW hypothesis using the VLF/LF Doppler observation (Asai et al., 2011). We have no definite observational evidence on the correlation between information near the Earth's surface and in the ionosphere, though we know very well that co-seismic ionospheric perturbations are apparently due to atmospheric oscillations (AW and AGW) (e.g., Davies and Baker, 1965; Weaver et al., 1970) because the focal zone of an EQ induces a strain process near the Earth's surface that would excite the AW and AWG propagating into the ionosphere. Korepanov et al., (2009) made the first attempt in this direction by making full use of the data of ground-based pressure and magnetic fields and satellite-based plasma, but with using meteorological disturbances, to indicate the importance of AGW channel for the LAI C. And, Nakamura et al., (2013) have extended the idea of Korepanov et al., (2009) to pre-EQ effects, who have indicated some possible relationship between the Earth's surface change and the ionosphere. Recently Yang et al., (2019) have provided the first convincing evidence on the presence of AGWs in the atmosphere with the use of satellite data for a particular EQ (the 2016 Kumamoto EQ). Further detailed studies are required in order to acquire observational facts in favour of the second channel.

An alternative hypothesis (electrostatic channel) has also been suggested by Freund (2009), who summarized his own results based on laboratory experiments. The discovery of positive holes charge carriers in crustal rocks, alongside electrons, opens a window of opportunity to study all these pre-EQ signals. He shows that pre-EQ signals are the consequence of one and the same process: stress activation of electrons and positive holes in rocks. When the positive holes arrive at the Earth's surface, they can cause a variety of effects including ionization of air at the ground-air interface, perturbations in the ionosphere, and distinct infrared (IR) emissions. When and how electric currents flow deep within the crust depends on the flow pattern. Under certain conditions, probably late in the EQ preparation process, the "battery circuit" can close. In this case, the electric currents flowing out of the stressed rock volumes can become very large, potentially on the order of millions of amperes, leading to powerful ELF/ULF emissions, but the penetration of electric field into the ionosphere meets the same difficulty as in the case of the first channel.

Further, Sorokin and Hayakawa (2013) checked the previous theories (channel (1) of Pulinets and Boyarchuk (2004)) and have proposed a new modelling of the influence of EQ preparation processes on the ionosphere through the electric field and electric current occurring in the global atmosphere- 
ionosphere electrical circuit. Their model is based on the generation of electric fields as a result of injection of charged aerosols into the atmosphere (i.e., electromotive force (EMF)), which could explain the generation of electric field within the ionosphere without any significant electric field near the Earth's surface.

When we have a sufficient intensity of electric field in the ionosphere, it would lead to different kinds of perturbations or instabilities (Pulinets and Boyarchuk, 2004; Sorokin and Hayakawa, 2013), as shown in Figure 42. Though the details are rather complicated, the generation of such an EMF might result in AGW instability in the ionosphere, the formation of field-aligned current and plasma irregularities, and many other related phenomena. The generation of the well- defined whistler ducts evidenced in Section 5.7 may be explained successfully in terms of the earlier instability.

\subsection{Modeling of anomalies in SR}

The temporal evolution of anomalies in SR observed in Japan, in possible association with EQs in Taiwan as shown in Section 3, is quite similar to that of seismo-ionospheric perturbation as detected from our VLF/LF observations. We turn to an impact of such an ionosphere perturbation over Taiwan during the Chi-chi EQ on the SR records in Japan.

ELF propagation including SR and ULF/ELF transients is connected to two characteristic heights $\left(\mathrm{h}_{1}\right.$ and $\left.h_{2}\right)\left(\right.$ Greifinger and Greifinger, 1978). The first altitude $h_{1}$ is regarded as the electric height where the displacement current at a given frequency becomes equal to the conductivity current. It is the height where the atmosphere turns from a neutral to conducting medium. The electric field rapidly decreases above the $h_{1}$ altitude. The $h_{2}$ is the magnetic height where radio propagation changes from the wave-like to the diffusion-like. Only the magnetic field component reaches the altitude $\mathrm{h}_{2}$. The characteristic value of $\mathrm{h}_{1}$ at SR frequencies is $\mathrm{h}_{1} \sim 55 \mathrm{~km}$, while $\mathrm{h}_{2} \sim 95 \mathrm{~km}$ (Nickolaenko and Hayakawa, 2002, 2014; Mushtak and Williams, 2002). Therefore, we note that the height range relevant to ELF propagation is definitely lower than the VLF reflection height, and it is also perturbed by seismic activity. The scale of the perturbation in the ionosphere is estimated by the radius $R($ in $\mathrm{km})=\exp (\mathrm{M})$ (Ruzhin and Depueva, 1996), which yields $\mathrm{R} \sim 2000 \mathrm{~km}$ for the magnitude of the Chi-chi EQ (M=7.6). This means that seismoatmospheric and seismo-ionospheric perturbations have the spatial scale of $1 \mathrm{Mm}$ at least. The ionosphere non-uniformities must reflect the ELF waves from the area above the epicenter of the Chi-chi EQ (in 
Taiwan). Liu et al., (2001) have studied the F region ionosphere modifications during the Chi-chi EQ and have found that the precursors appeared 1-6 days prior to the EQ major shock. This means that similar anomaly definitely occurs in the lower ionosphere.

We suggest the following explanation based on the wave interference. The two characteristics of SR anomaly are important: (1) the fourth resonance peak is noticeably enhanced in the By field component and (2) a significant shift occurs in the peak frequency of this component reaching $1 \mathrm{~Hz}$. One must take into account that the major field sources during observations of SR anomaly were the thunderstorms in the South-East Asia $\left(0^{\circ} \mathrm{N}\right.$ and $\left.120^{\circ} \mathrm{E}\right)$, Africa $\left(5^{\circ} \mathrm{N}\right.$ and $\left.10^{\circ} \mathrm{E}\right)$, or in the South America $\left(0^{\circ} \mathrm{N}\right.$ and $\left.50^{\circ} \mathrm{W}\right)$.We also suggest that the conductivity of lower ionosphere increases substantially above the epicenter of EQ in Taiwan, and that this non-uniformity acts as an ELF wave reflector. We use in the model computations one of three global thunderstorm centers positioned in Asia, Africa, and America. The configuration is shown in Figure 43 of propagation paths relevant to direct and the reflected waves arriving from one of thunderstorm centers and the scatterer at Taiwan. In the case of the Asian source, the source-observer distance is $5.5 \mathrm{Mm}$, and the path difference between the direct wave and the wave scattered at Taiwan is about 5.9 $-5.4=0.4 \mathrm{Mm}$. The maximum effect (the strongest wave interference) is expected when this path difference is equal to $\lambda / 2$ ( $\lambda$ is the wavelength). Hence, one obtains $\lambda=0.8 \mathrm{Mm}$ for the expected maximum interference. Since $\lambda=40 \mathrm{Mm}$ at the first SR mode, the above wavelength corresponds to the mode number equal to $40 / 0.8=50$, or to the frequency of approximately $300 \mathrm{~Hz}$. Hence, the Asian thunderstorms are the poorest candidate for the strong wave enhancement at the fourth SR mode frequency.

Let us test the African thunderstorms. The source-observer distance is about $13 \mathrm{Mm}$, and the path difference between the direct and the scattered waves is $\sim 2 \mathrm{Mm}$. The maximum effect might be expected at $\lambda=4 \mathrm{Mm}$. This wavelength corresponds to the mode number equal to $40 / 4=10$, or to the frequency of about $60 \mathrm{~Hz}$. So, the thunderstorms in Africa are not excellent, but a realistic candidate. The third source candidate is South America. The relevant source-observer distance is about $19 \mathrm{Mm}$ and the path difference between the direct and scattered waves is $4 \mathrm{Mm}$. One can expect that the maximum wave interference will be observed at wavelength $\lambda=8 \mathrm{Mm}$. This wavelength corresponds to the mode number $40 / 8=5$, or to the frequency of approximately $32 \mathrm{~Hz}$. In this sense, the American source positioned at the Amazon basin is the most reasonable candidate for the model explanations. Besides, the direction from Taiwan and from Nakatsugawa (NAK) toward the Amazon basin are nearly 
coincident, the direct and the scattered wave arrival azimuths are separated by $\sim 180^{\circ}$, and we have the situation of wave backscatter. The observer receives the direct signal from the American source and the signal reflected from the ionospheric perturbation located at Taiwan arrives from the opposite direction (see Figure 43).

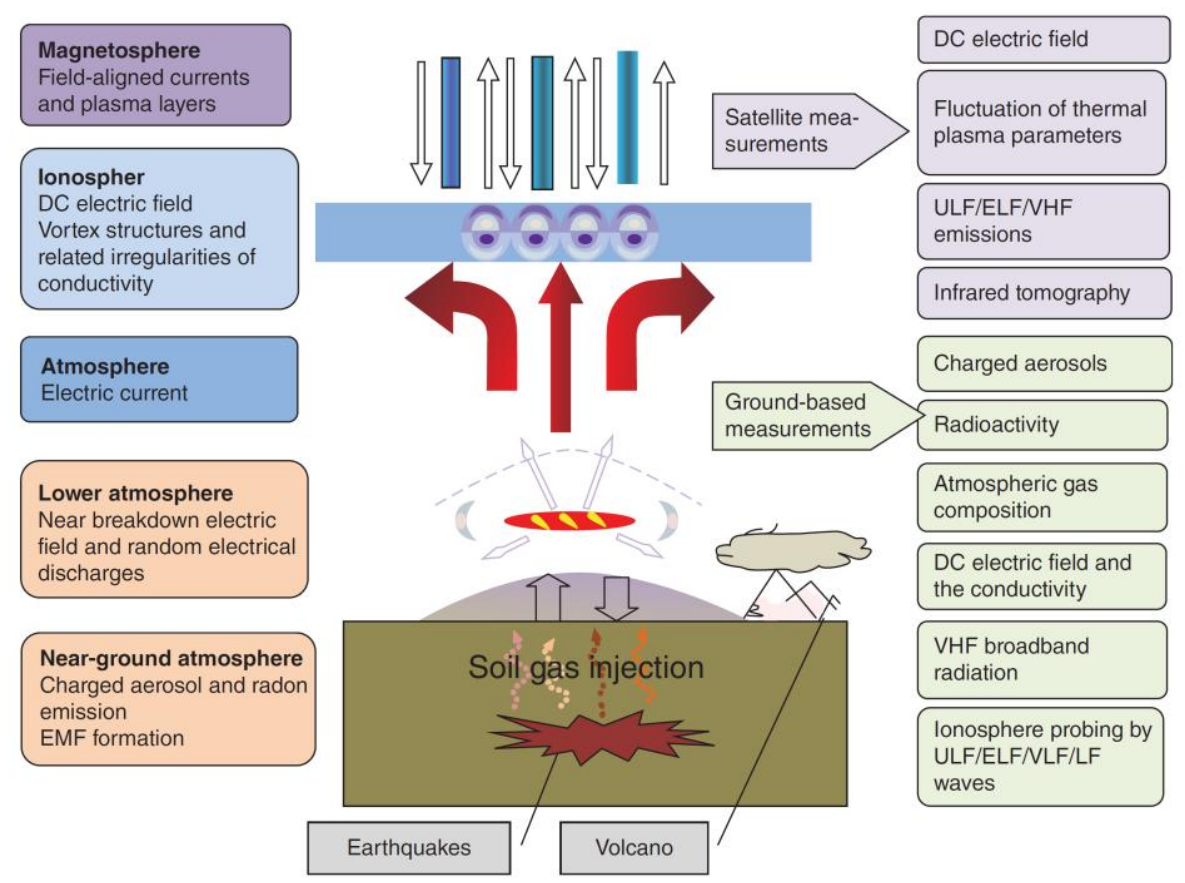

Figure 42: The schematic picture of electrodynamics model of LAIC, with possible different kinds of plasma and electromagnetic effects. Reproduced from Sorokin and Hayakawa (2013) with permission from Modern Applied Science.

We address the scattering problem for different lightning sources shown in Figure 43 and the ionosphere disturbance located at Taiwan. The details were might be found in Hayakawa et al., (2005) and Nickolaenko et al., (2006) .We use the following model parameters in our computations. The observer is located at NAK, Japan $\left(35.45^{\circ} \mathrm{N}\right.$ and $\left.137.3^{\circ} \mathrm{E}\right)$. The regular lower ionosphere is described by the knee model (Mushtak and Williams, 2002) having the electric characteristic height h1 $55 \mathrm{~km}$ and 


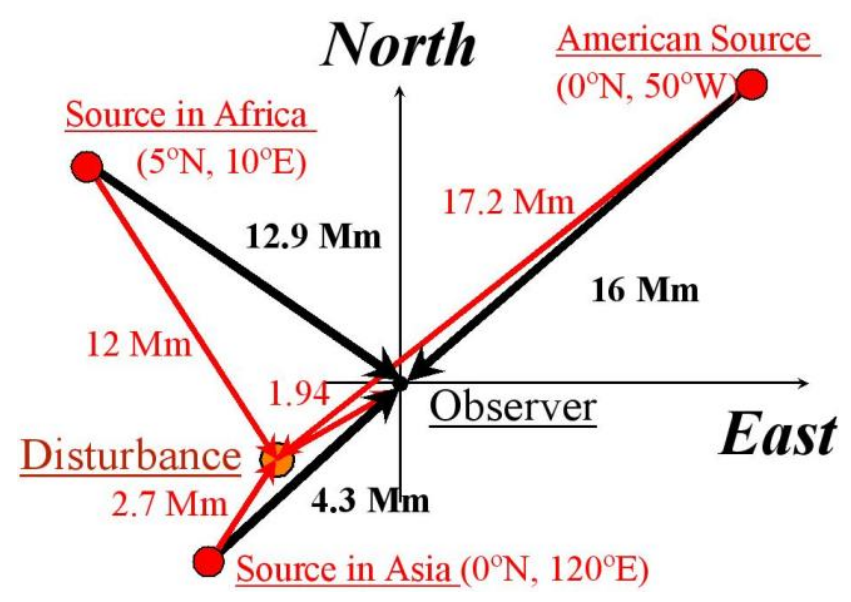

Figure 43 : Three thunderstorm regions, (a) Asia (Indonesia), (b) Africa and (c) America (Amazon) and the configurations of the direct path and that scattered at Taiwan are given for each source.

the magnetic characteristic height $h_{2} \sim 95 \mathrm{~km}$. The regular propagation constant $v(f)$ is found from the relation: $v(v+1)=(k a)^{2} h_{2} / h_{1}$, where $a$ is the Earth's radius and $k$ is the wave number. The vertical electric field component $E_{1}$ of the direct wave arriving at the observer from the vertical dipole source is equal to: $E_{1}=\frac{M(\omega)}{4 h_{1} a} \frac{i v(v+1)}{\omega} \frac{P_{v}\left[\cos \left(\pi-\theta_{H}\right)\right]}{\sin v \pi}$. Here, $M(\omega)$ is the current moment of the source, $P_{\downarrow}(x)$ is the Legendre function of complex order $v$, and $\theta_{\mathrm{H}}$ is the source - observer angular distance.

The disturbance in the atmosphere conductivity profile is characterized by a reduction of the lower part of atmosphere conductivity profile by $20 \mathrm{~km}$. Therefore, its lower (electric) characteristic height $h_{1}$ is reduced by $20 \mathrm{~km}: h_{\mathrm{D}}=h_{1}-20 \mathrm{~km}$. The disturbance is placed over Taiwan with geographic coordinates $\left(24^{\circ} \mathrm{N}\right.$ and $\left.122^{\circ} \mathrm{E}\right)$, and the modification of the dimensionless propagation parameter $\Delta C_{v}^{2}=\frac{h_{2}}{h_{D}}-\frac{h_{2}}{h_{1}}$ reaches its maximum at this point. The disturbance amplitude varies along the radius $\rho$ according to the Gauss law: $\delta C_{v}^{2}=\Delta C_{v}^{2} \exp \left(\frac{\rho^{2}}{R^{2}}\right)$, where the horizontal characteristic size of perturbation is $R=1000 \mathrm{~km}$. The problem is solved by using the Stratton-Chu integral equation, and the field at the observatory is the sum of direct field $E_{1}$ and that scattered by the non-uniformity wave $E_{2}$ (Nickolaenko and Hayakawa, 2002, 2014; Hayakawa et al., 2005). The relative disturbance of electric field amplitude is equal to: 
$B=\frac{E_{2}}{E_{1}}=\frac{\Delta C_{v}^{2}\left\lceil Q_{v} \sin \theta d \theta d \varphi\right.}{4 \sin \pi v P_{v}\left[\cos \left(\pi-\theta_{S}\right)\right]}$ where $\theta_{S}$ is the distance from the observer to a particular thunderstorm center and $Q_{v}=v(v+1) P_{v}[\cos (\pi-\theta)] P_{v}[\cos (\pi-\gamma)]-\frac{\partial}{\partial \theta} P_{v}[\cos (\pi-\theta)] \frac{\partial}{\partial \gamma} P_{v}[\cos (\pi-\gamma)]$. When obtaining these equations we took into account that a localized non-uniformity acts as a delta-function in the 2D (two dimensional) Stratton-Chu surface integral. The physical meaning of the obtained formula is clear: the field scattered by non-uniformity contains the product of two Green's functions. One describes the wave arriving from the source to the non-uniformity, and it has the $(\pi-\theta)$ argument being the angular distance between the source and non-uniformity. The second term corresponds to the scattered wave, and it depends on the observer - non-uniformity distance relevant to the argument $(\pi-\gamma)$. Since the electromagnetic fields have the vectorial nature, the solution contains both Green's functions and their derivatives. In the case of scattering the scalar waves, e.g., the acoustic waves, the solution does not contain the derivatives of Green's functions.

We used in our computations three global thunderstorm centers driving the SR signal, which occupy the following positions: $\left(0^{\circ} \mathrm{N}\right.$ and $\left.120^{\circ} \mathrm{E}\right)$ - South-East Asia; $\left(5^{\circ} \mathrm{N}\right.$ and $\left.10^{\circ} \mathrm{E}\right)-$ Africa; $\left(0^{\circ} \mathrm{N}\right.$ and $\left.50^{\circ} \mathrm{W}\right)-$ South America. Our goal was to establish whether a localized disturbance over Taiwan might produce an effect similar to that observed experimentally. We also expected to observe an impact of the source position on the resulting field modifications. The particular value of disturbance and relevant type of ionosphere modification could be addressed later in a more detailed comparison of special computations and measured data.

The results of computations are shown in Figure 44. The left frame illustrates the frequency dependence of the amplitude $|B(f)|$ of dimensionless field disturbance, computed for the point sources located in Asia (dotted line), Africa (smooth line), and America (dashed line). One may see that the highest relative disturbance is associated with the Asian source, and it is observed around the $20 \mathrm{~Hz}$ frequency or the third SR mode. The plots in the left frame of Figure 43 provide a "general picture" of the wave scattering. One must have in mind that the large values of $|B(f)|$ function might correspond not only to a high scattered field, but also to the small direct wave arriving at the observatory, e.g. at the nodal source - observer distance. As the lower frame indicates in the right column of the plots in Figure 44, this situation takes place for the Asian source. 
The right plots in Figure 44 depict the model amplitude spectra of vertical electric field component when the Earth - ionosphere cavity is excited by thunderstorms in America, Africa, or Asia (from top to bottom). The smooth black lines in these plots show the spectra of the direct wave (in the absence of ionosphere non-uniformity); they are marked by the "Direct wave" label text. The dashed lines in these frames show the model SR spectra in the presence of the ionosphere modification, marked as "Disturbed". The lower right frame depicts the data computed for the Asian source. One may see that the undisturbed field has a minimum around the $20-\mathrm{Hz}$ frequency. This is why a large peak is present in the left frame for the Asian source. The model spectra show that this source might produce a noticeable disturbance only around the second SR mode.
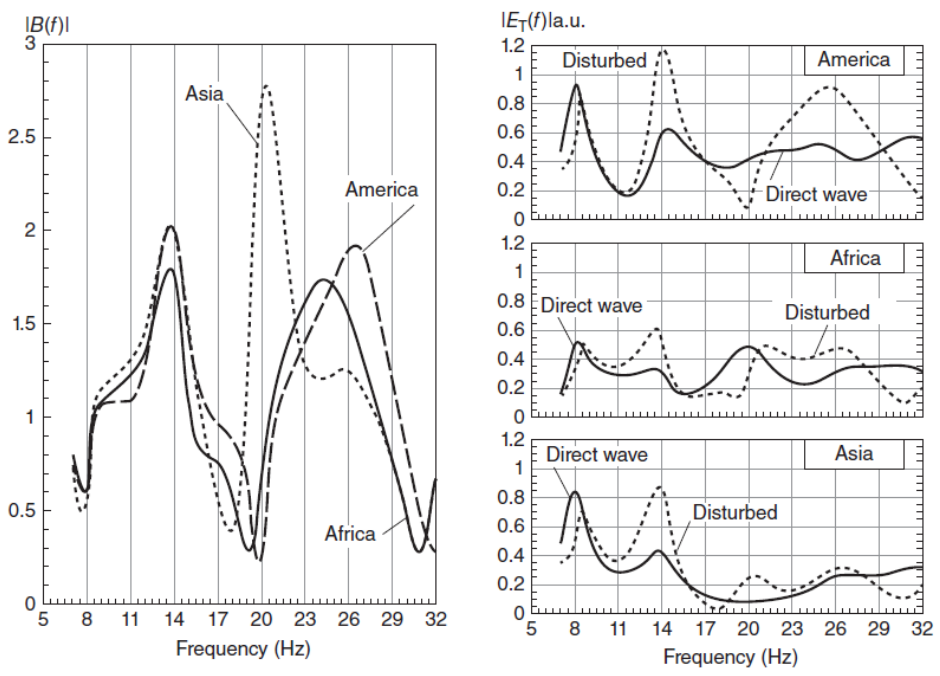

Figure 44 : Left panel shows the computational results on the frequency dependence for three thunderstorm centres. The right panel indicates the frequency dependences of the vertical electric field expected at our observatory for three sources (from the top to the bottom; America, Africa and Asia). A thin line refers to the direct wave without the effect of ionospheric perturbation, while a thick line, the corresponding result for the disturbed case (with the ionospheric perturbation over Taiwan). After Hayakawa et al., (2005) with permission from EGU.

The model amplitude spectra in the presence of seismogenic non-uniformity indicate that American thunderstorms (Amazon basin) are positioned in such a way that a two-fold increase is observed in the spectral amplitude at frequencies around the fourth SR mode. The physical reasoning for such a feature was introduced before. The non-uniformity over Taiwan causes a smaller increase in the amplitudes of the waves arriving from other global thunderstorm centers. We may conclude that the presence of 
seismogenic ionosphere perturbation over Taiwan causes the specific interference between the direct and reflected wave, which results in an enhancement of the fourth SR mode amplitude when observed at the observatory of NAK. We must emphasize that the wave interference concept successfully explains two major points of experimental observations: the noticeable amplitude increase in the vicinity of the fourth SR mode and a simultaneous shift of the fourth peak frequency. As seen in Figure 44, the seismoionospheric perturbation is likely to influence the lower frequencies as well, but we do not address this here in more detail.

The SR anomalies were explained in terms of the wave interference based on the assumption that the ionosphere (mesosphere) conductivity is perturbed over the EQ epicenter. This idea was successfully applied for interpreting the SR data relevant to the 2011 Tohoku EQ, see, e.g. Ouyang et al., (2013). We exploit here the concept of atmosphere perturbation over the EQ epicenter or the LAIC mechanism; however, we did not address the particular process itself as it was indicated in Section 9.1.

\subsection{Possible interpretation of the ULF magnetic field depression}

We studied the fluctuations of geomagnetic fields in the frequency range of geomagnetic pulsations (0.01$0.1 \mathrm{~Hz}$ ) at middle and low geomagnetic latitudes in highly seismic regions of Kamchatka (Russia) and Japan in Section 4. The basic idea of the present phenomenon is that the parameters of natural ULF geomagnetic noise can change significantly near the epicenter of a forthcoming EQ due to the LAIC.

We have been treating nighttime ULF geomagnetic field variations, because the artificial (industrial) noise is minimum during nighttime, so that we deal with nighttime irregular pulsations Pis (e.g. Saito, 1969; Jacobs, 1970; Nishida, 1972; Alperovich and Fedorov, 2007; Surkov and Hayakawa, 2014). The fundamental basis for this phenomenon lies in the fact that the amplitude of ULF geomagnetic fluctuations depends both on the amplitude of incident wave and the parameters during their propagation in the magnetosphere and ionosphere. It is not reasonable to think about the EQ effect on the equatorial plane of the magnetosphere where those pulsations are mainly generated, so that it is quite natural to attribute some changes in the ground-based ULF noise to the change in the ionospheric parameter as in the case of EQ effect on Pc 1 pulsations (Section 5), because we already know that the ionosphere is definitely perturbed before major EQs. 
Numerous attempts were made to explain seismo-associated ULF phenomena with the direct emission from an EQ origin zone (e.g., Fenoglio et al., 1995; Molchanov and Hayakawa, 1995) or with the conductivity variations inside and near the EQ zone, leading to the variation in the amplitude of pulsations (e.g., Merzer and Klemperer, 1997). The first mechanism should result in the growth of ULF amplitudes, but actually the ULF horizontal magnetic field amplitude deceased before strong EQs as in Section 4. Variations of the ground conductivity are also doubtful because the long-term magneto telluric observations in USA and Japan did not show any correlation of the crust conductivity with seismicity (see, e.g., Park, 1997). So, Molchanov et al., (2004) suggested that the depression of magnetic pulsations is caused by any ionospheric disturbances before violent EQs in the following.

We shall consider two models to explain the mid-latitude ULF geomagnetic noise depression:

1. The source is far above the ionosphere, i.e. in the magnetosphere. The ionospheric influence is through the variations of transmission coefficient as Model I in Figure 45.

2. The variation of the wave vector $(\mathbf{k})$ of the ionospheric fluctuations as Model II in Figure 45.

For the first scenario (Model I), incident "noise" (broadband) as hydro magnetic waves fall onto the ionosphere, which are registered on the Earth's surface as natural ULF geomagnetic noise in Figure 45.The incident ULF wave from above is likely to be Alfven waves (A-waves) (ion Cyclotron waves), so let us consider the penetration of Alfvén waves to the ground observer. The transmission coefficient $\mathrm{T}_{\mathrm{A}}$ of the incident A-waves is controlled by the ratio of the ground magnetic field $b_{A}^{(g)}$ to the amplitude of the incident Alfven wave $b_{A}^{(i)}, T_{A}=b_{A}^{(g)} / b_{A}^{(i)}$. One can get the following formula for $\mathrm{T}_{\mathrm{A}}$ (Hughes and Southwood, 1976; Alperovich and Fedorov, 2007).

$$
\mathrm{T}_{\mathrm{A}} \approx \frac{2 \sum_{\mathrm{H}}}{\sum_{\mathrm{P}}+\sum_{\mathrm{A}}|\sin \mathrm{I}|}
$$

where $\Sigma_{A}=1 / \mu_{0} V_{A}$ is the Alfvén wave conductance, $V_{A}$ is the Alfvén velocity, $\mu_{0}$ is free space permittivity, and I is the angle between the geomagnetic field and the horizontal plane. Note that the ratio of the ground wave amplitude to that of the incident wave should be used, but not the often used 
ratio of the ground to full wave (incident plus reflected) $b_{A}$. The well-known expression $b_{A}^{g} / b_{A} \propto \Sigma_{H} / \Sigma_{P}($ Hughes and Southwood, 1976) is written for the full wave amplitude.

The ionospheric screening grows with an increase in Pedersen conductivity or a decrease in Hall conductivity. Typical values of night-time integral conductivities $\Sigma_{P} \sim \Sigma_{H} \sim 10^{-1} \mathrm{~S}$ and $\Sigma_{A} \approx 1 \mathrm{~S}$. At nighttime $\Sigma_{P}$ is formed predominantly at altitudes $120-200 \mathrm{~km}$ and $\Sigma_{H}$ at $100-120 \mathrm{~km}$.

Let us consider first the decrease of transmission coefficient because of Pedersen conductivity. The Pedersen conductivity can increase without any essential growth of Hall conductivity if the plasma concentration grows at altitudes $120-200 \mathrm{~km}$. However, for a noticeable decrease in $\mathrm{T}_{\mathrm{A}}$ Pedersen conductivity should be at least as big as $\Sigma_{A}|\sin I| \approx 1 \mathrm{~S}$. This corresponds to the growth in electron concentration by 5-10 times at 120-200 km. Thus, this scenario might not be unreasonable.

However, there may be an alternative mechanism for the increase in Pedersen conductivity. The contribution of electrons to $\Sigma_{P}$ is usually negligible in the ionosphere, but the role of electrons becomes important in the inhomogeneous ionospheric plasma. If the level of fluctuations in the ionospheric plasma increases before an EQ, this leads also to the growth in Pedersen conductivity. We use the formula for a rough estimate (Dreizin and Dykhne, 1973; Alperovich and Chaikovsky, 1995),

$$
\sigma_{x x}^{e}=1.3 \cdot 10^{-11} \gamma^{4 / 3} N_{e} v_{e}^{1 / 3} \quad \mathrm{~S} / \mathrm{m}, \quad \gamma \beta_{e}>>1,
$$

where $\gamma=\frac{\left\langle\delta N_{e}^{2}(\mathbf{r})\right\rangle^{1 / 2}}{\left\langle N_{e}(\mathbf{r})\right\rangle}, \quad \delta N_{e}(\mathbf{r})=N_{e}(\mathbf{r})-\left\langle N_{e}(\mathbf{r})\right\rangle, \quad \beta_{e}=\omega_{H} / v_{e}, N_{e}$ is electron concentration, $v_{e}$ is the frequency of electron collisions and $\omega_{\mathrm{H}}$, electron gyro frequency. A rough upper estimate by Eq. (7) even at $\gamma=1$ gives a slight increase in $\Sigma_{P}$, but not exceeding several percent of $\Sigma_{A}$.

We now consider the decrease of Hall conductivity. For $\Sigma_{P}<<\Sigma_{A}|\sin I|$, TA is proportional to the Hall conductivity, because the influence of Pedersen conductivity is negligible. This means that the transmission coefficient $T_{A}$ is proportional to the total electron concentration at $100-120 \mathrm{~km}$. Thus if the source of geomagnetic fluctuations lies in the magnetosphere, the ionosphere can cause the depression of ULF geomagnetic fluctuations only under the decrease in total electron concentration at 100-120 km. We then discuss another possibility with the ionospheric source of the geomagnetic fluctuations (Model II). We assume the electric fields $\mathrm{E}^{(\mathrm{i})}$ to have random phases $\left(\left\langle\Delta \mathrm{E}^{(\mathrm{i})}\right\rangle=0\right)$. The coordinate system is 
chosen Cartesian with upward vertical z-axis. The main geomagnetic field is $\mathbf{B}_{\mathbf{0}}=\mathrm{B}_{0} \mathbf{z}$. The electromagnetic field is presented as a sum of harmonics $\propto \exp \left(-i \omega t+i k_{\perp} r\right)$, where $x$-axis is directed along $\mathrm{k}_{\perp}$ and $\mathrm{k}_{\mathrm{x}}=\mathrm{k}$. Fluctuations are assumed to be isotropic, and then taking into account the dispersion equation $\omega=\omega(\mathrm{k})$ one gets the magnetic field on the Earth's surface,

$$
\left|b_{x}^{(g)}(\omega)\right|^{2}=\int \mathrm{T}^{(\mathrm{i})}(\mathrm{k}) \frac{\left|E_{x}^{(i)}(k)\right|^{2}}{V_{A}^{2}} \mathrm{dk}
$$

Where $T^{(i)}=T_{x}^{(i)}+T_{y}^{(i)}, T_{x}^{(i)}, T_{y}^{(i)}$ are the penetration coefficients for the normalized electric field of the ionospheric fluctuations. Molchanov et al, (2004) assumed that

$$
\left|E^{(i)}\right|^{2} \propto \frac{K^{2}}{K *^{2}}\left(1+\frac{k^{2}}{k *^{2}}\right)^{1 / 6}
$$

This reduces to the classical Kolmogorov's distribution $\mathrm{k}^{-5 / 3}$ for $\mathrm{k}>>K_{*}$, where $K_{*}$ is inverse scale $\left(L_{*}\right)$ of the external source. Molchanov et al. (2004) used $K_{*}=10^{-3} \mathrm{~km}^{-1}\left(L_{*} \sim 1000 \mathrm{~km}\right)$ for quiet situation and $K_{*}=5 \cdot 10^{-3} \mathrm{~km}^{-1}\left(L_{*} \sim 200 \mathrm{~km}\right)$ if the main source is AGW induced by seismicity. Then Molchanov et al,. (2004) obtained the nearly two-fold decrease of the penetration coefficient $T^{(i)}$ in the ULF frequency range, seeming to be consistent with the observation in Section 5.

Thus the most reliable mechanism for depression of background ULF activity (horizontal magnetic fields) must be located somewhere in the lower ionosphere whatever the mechanism is either as any change in transmission coefficients of magnetospheric waves (Model I) or as the decrease of spatial scales of ionospheric sources caused be seismo-induced AGW (Model II). Anyhow further detailed quantitative estimations for different possibilities are highly required before the final conclusion on the mechanism is reached.

\subsection{Modeling of EQ effect on Pc1 pulsations}

Pc1 in the frequency range of $0.2-5 \mathrm{~Hz}$, has been identified over 40 years that they are one of the most well-known magnetospheric plasma waves (Saito, 1969; Jacobs, 1970; Nishida, 1972). It is found that 
Pc pulsations are generated only at high latitudes by plasma instability in the equatorial plane, then have propagated along the magnetic field in Alfven (ion Cyclotron) mode (L mode) penetrated through the ionosphere, to be observed on the ground (Saito, 1969; Alperovich and Fedorov, 2013; Surkov and Hayakawa, 2014). A schematic picture of the generation and propagation of Pc 1 is given in Figure 29 reproduced from Bortnik et al., (2008). In the ionosphere those L mode waves undergo the mode conversion, and excite magnetosonic $\mathrm{R}$ waves and they propagate in the ionospheric waveguide peaked at the $\mathrm{F}$ region from high to low latitudes, and some leakage to the ground as low latitude Pc1 pulsations. So the detection of Pc 1 pulsations at a low-latitude ground station is strongly dependent on the condition of such an ionospheric waveguide (as in Figure 29 (b)), especially lower boundary of the waveguide as in the case of previous subsection. Because it is quite unreasonable to expect the EQ effect on the excitation mechanism of Pc 1 pulsations deep in the magnetosphere, it seems more natural to suppose any kind of changes in ionospheric parameters during the pre-EQ process as in previous subsections of 9.2 and 9.3.It is recently well established as is shown in Section 9.1.thatthe lower ionosphere is evidently perturbed about one week before an EQ (e.g., Hayakawa et al., 2010, 2018), and correspondingly we expect some significant change in the ground reception of Pc 1 waves. Bortnik et al., (2008) only suggested a hypothetical interpretation that the ionosphere above the EQ epicenter is modified in such a way that something like a plasma lens appears, which enhances the infiltration of Pc 1 waves from the ionospheric waveguide to the ground.

The leakage mechanism of Pc 1 pulsations in the disturbed ionospheric waveguide should be extensively investigated because no papers on the waveguide propagation of Pc 1 pulsations have been published for the disturbed ionosphere. It is highly required to perform extensive theoretical works on the penetration to the ground of isotropic magnetosonic $\mathrm{R}$ waves propagating in the disturbed waveguide, especially on the question of which parameter (region) of the ionospheric waveguide would play the key role in the change of characteristics of ground-based Pc 1 pulsations (such as daytime enhancement before an EQ as observed by Bortnik et al., (2008)).

Guglielmi and Zotov (2010) have studied the correlation between Pc 1 pulsations and EQs, with special attention to co-seismic effects and they have found a significant negative correlation between the two. Their speculation on the EQ effect on Pc 1 pulsations is as follows. The co-seismic effect is the action of the acoustic gravity waves (as already well documented by the observations (e.g., Davies and Baker (1965) and others)), which reduces the reception of ground-based Pc 1 pulsations though the 
micro-mechanism is not well presented. They also try to apply this concept even to pre-EQ effects as in this paper, but it is open for discussion. Similar much more observational works will be highly required at different latitudes in the world, together with theoretical modelings.

\subsection{Modeling of seismogenic ULF/ ELF emissions (or Q bursts)}

Concerning the generation mechanisms of seismogenic atmospheric Q bursts, we have discussed two possibilities closely related with LAIC or exactly the same as LAIC: (i) perturbations of the electric field induced by the pre-seismic ionized gas release (e.g. radon), and (ii) AGWs or the infrasound turbulence excited by sporadic water/gas eruptions or by foreshocks and aftershocks during a time interval about 2 weeks around the EQ time. However, if we pay more attention to the Fujinawa'soccasional position of the perturbed region over the sea at the off shore distance $\sim 100 \mathrm{~km}$ (though not well established), the first possibility looks less probable than the second. The first process causes the discharges in the atmosphere, seeming to be consistent with the observation, and so more extensive works are also required on this mechanism.

Marie et al., (2002) and Molchanov et al., (2004) considered the second possibility and showed that for the typical periods $0.5-1$ hours and horizontal scales of the disturbances of about $\lambda \sim 1 \mathrm{~km} \mathrm{AGWs}$ propagate in the direction about $5^{\circ}-7^{\circ}$ to the horizon. For distances $100-200 \mathrm{~km}$ corresponding altitudes are 10-20 km, and the resulting density fluctuations are about $1 \%$. But the principal question about the mechanism of generating observed phenomena due to low amplitude disturbances is still unclear.

The third mechanism is also associated with the enhanced plasma concentration above the EQ preparation zone and thus the disturbance of the conductivity $(\sigma) \Delta \sigma$. It is well known that the Earthionosphere waveguide is filled with electromagnetic emissions from thunderstorms (Nickolaenko and Hayakawa, 2002) and the observed pre-seismic emission can be the waves scattered from the ionospheric inhomogeneity. Let a typical scale of the disturbance be small in comparison with the radius a. In a periodical electric field $\mathrm{E}_{0} \propto e^{-i \omega t}(\omega=2 \pi f, \omega$ is angular frequency, and f frequency), the disturbed sphere obtain an electric moment $\mathrm{P}_{0}=\mathrm{a}^{3} \Delta \sigma / \sigma \mathrm{E}_{0}$ if $\Delta \sigma<<\sigma$, and the corresponding current moment $\mathrm{M}_{0}=i \omega \mathrm{P}_{0}=-2 \pi \mathrm{ifa}^{3} \Delta \sigma / \sigma \varepsilon_{0} \mathrm{E}_{0}\left(\varepsilon_{0}\right.$, free space dielectric constant). At $\rho>\mathrm{h}$ where $\mathrm{h}$ is the 
waveguide thickness and $\rho$ is the distance from the center of the disturbed sphere to the observational point, only the magnetic field of the $\mathrm{TM}_{0}$ is sufficient. It can be written as,

$$
b_{\varnothing}=\frac{\mu_{0}\left|M_{0}\right|}{4 \pi} \frac{1}{h \rho}=\frac{\Delta \sigma}{2 \sigma} \frac{f a^{3}}{h \rho c} \frac{E_{0}}{c} \text { (c=light velocity). }
$$

Substituting $\mathrm{E}_{0} \approx \mathrm{b}_{0} \mathrm{c}$ (where $\mathrm{b}_{0}$ is the typical magnitude of the magnetic field) into Eq. (10), we obtain

$$
\frac{b_{\emptyset}}{b_{0}}=\frac{f a^{3}}{2 h p c}=\frac{\Delta \sigma}{\sigma}
$$

At $\mathrm{f}=5 \mathrm{~Hz}, \mathrm{a}=50 \mathrm{~km}, \mathrm{~h}=50 \mathrm{~km}, \rho=200 \mathrm{~km}$, we obtain from Eq. (11) $b_{\varnothing} / b_{0} \sim 10^{-4} \Delta \sigma / \sigma$.Even for $\Delta \sigma / \sigma=$ 1 the radio of scattered to incident magnetic fields is too small to cause the observed effect.

As the conclusion, the mechanism of this seismogenic atmospheric ULF/ELF impulsive signal is extremely poorly understood, and so further theoretical modeling is highly required.
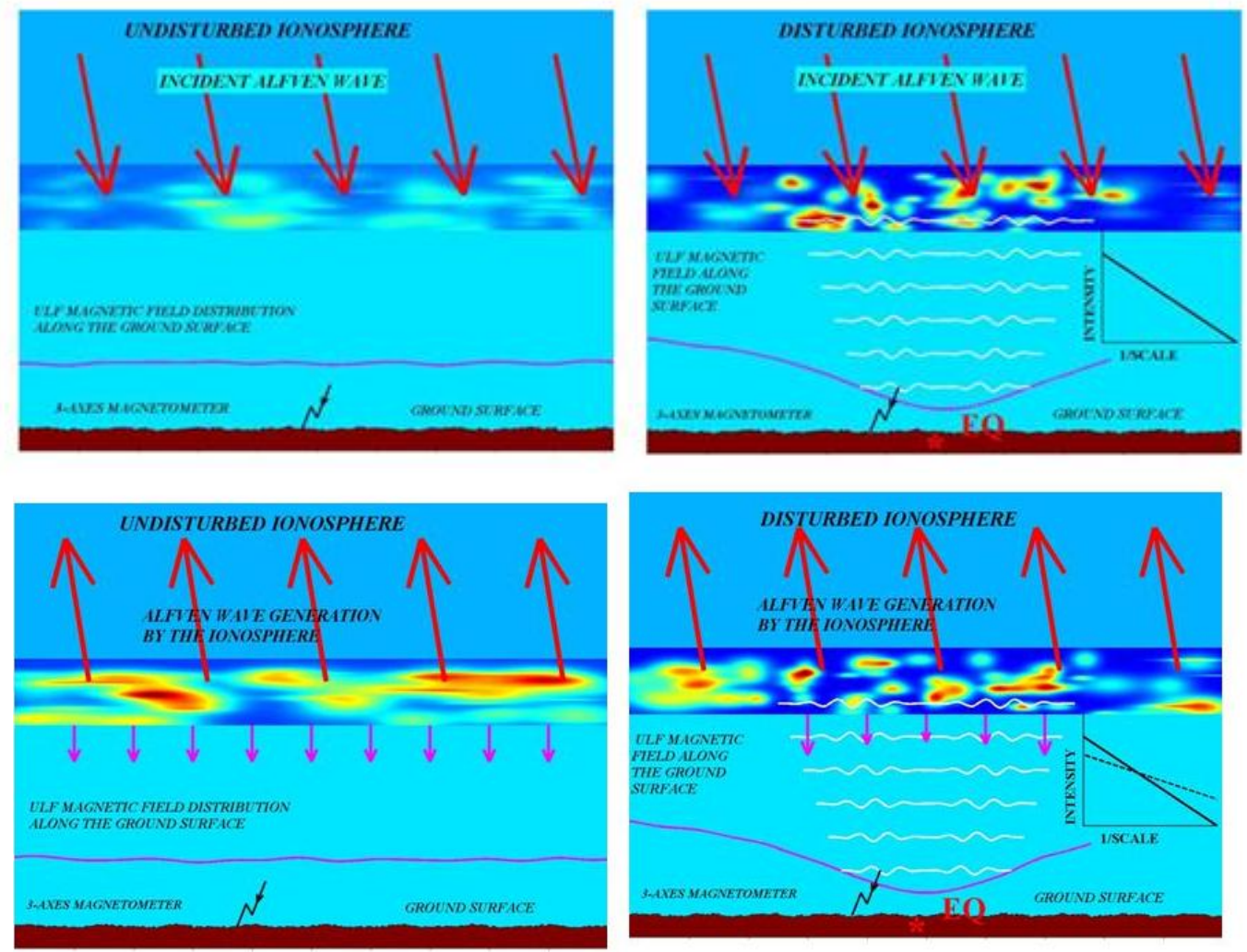

Figure 45: Upper panels: Model I on the decrease of the penetration coefficient of ULF magnetospheric pulsations due to intensification of the ionospheric disturbance. The left panel refers to the undisturbed condition, and the right, the disturbed ionosphere. Bottom panels: Model II on the decrease of the ULF magnetic field from the ionospheric source due to the scale re-distribution of the ionospheric turbulence in connection with an EQ. After Schekotov et al., (2013). 


\subsection{VLF electromagnetic emissions and lightning}

The nature of VLF emissions in possible association with EQs described in Sections 6 and 8 is not well established. That is, there seem to exist two different types. One is the impulsive type like lightning discharges, and the other is of noise type. Fujinawa et al., in Section 6 mentioned that the seismogenicVLF noise is of impulsive type and Liu et al. in Section 8 is definitely of impulsive type. However, some of the noise reported in Singh et al., is noise-like.

First we pay attention to the mechanism of impulsive noises in association with EQs. Liu et al., (2015) have presented the statistical results on the enhancement of lightning activity associated with EQs, but there have been already suggested some possible explanations on this topic.

Hayakawa et al., (2002) have proposed the following speculation. The main idea is as follows. Distribution of the electric field around a thundercloud depends on the conductivity profile of the atmosphere, so that such quasi-static fields seem to decrease in the regions with enhanced air conductivity. Air conductivity may grow due to pre-seismic gas and water releases into the atmosphere, as is already mentioned as one of the LAIC process (as discussed in Section 9.1). Then, the electric field becomes reduced in the lower section of troposphere, and the probability decreases of the cloud-to-ground stroke in such "contaminated" areas. Simultaneously, the field grows inside and above the thunderclouds, and hence the number of horizontal and tilted inter-cloud (or intra-cloud) strokes is expected to grow. Spatial redistribution of lightning strokes reduces their individual amplitudes, while the rate (median number strokes per a unit time) of discharges may grow. We demonstrate that transformation of strokes modifies the spectral content of ELF-VLF radio noise and changes the rate of detected pulses. We should know much more on the detailed characteristics (nature) of VLF waves and lightning. Together with this, different ideas should be pursued extensively in the context of atmospheric global circuit.

As for the noise-like VLF emissions, they are likely to be the background noise. But there have been proposed no ideas on their generation mechanism, and a further extensive work is required.

\subsection{EQ effect on $V L F / E L F$ whistlers}

Observational findings in Section 7 seem to indicate that the abnormal dispersion whistlers at a Japanese station of Sugadaira are correlated with EQs in the Japanese longitude. Of course, it is needless to say that 
much more observational studies are highly required at different places (or latitudes) of the world to have more evidence (or no such a correlation).

Here we have to confirm that the path latitude of whistlers with abnormal dispersions is found to be close to the latitude of our observing station in Japan. As mentioned in Section 7, the reception of abnormal dispersion whistlers is influenced by the propagation features in the magnetosphere, probably the presence of stable field-aligned irregularities (ducts) in the magnetosphere (as the first importance) and the source effect (lightning discharges in the opposite hemisphere) (as the second importance). Unlike the case of EQ effect on Pc 1 pulsations, these two effects seeming to be associated with EQs, are involved in the reception of whistlers, so it seems very difficult for us to think about the mechanism.

First we study the more important propagation effect. Low-latitude whistlers must have suffered from severe conditions during the course oftheir propagation (Hayakawa and Tanaka, 1978): (1) trapping in the duct at its entrance, (2) enhanced occurrence of ducts or very favourabl econditions of ducts in the magnetosphere, (3) transmission onto the ground at the receiver's site. As shown in Hayakawa et al., (1993), the EQs took place relatively close to the region of entrance of whistlers into the magntospheric ducts, and so the factors (1) and (2) are thought to be disturbed favourably, e.g. by AWs or AGWs generated as pre-EQ effect (see Figures 41 and 42) in such a way that a well-defined duct is formed and then duct trapping becomes much more favourable than usual. Alternatively, as shown in Figure 41, ULF waves are excited before an EQ in its focal zone, and they modify the ionospheric plasma as Alfven waves. Or, it may be possible that electric fields of thunderstorms (no matter whether these are the sources of whistlers) penetrating into the magnetosphere, may contribute significantly to the formation of well-defined ducts as initially suggested by Park and Helliwell (1971) and reconsidered by McCormick et al. (2002). In this scenario we have to consider the source effect as well, such as some relation of lightning activity in the equatorial and low latitudes with seismicity. That is, we may expect a higher lightning activity with seismicity as discussed in Sections 6 and 8, even though we do not know whether this lightning is the same one as that considered for duct formation.

As the conclusion, even though the statistical relation of the occurrence of whistlers (with abnormal dispersions) in Japan and EQs in the Japanese longitude is likely, the mechanism on the EQ effect on whistlers is much more puzzling than the EQ effect on Pc 1 pulsations in Section 9.4., and is open for future discussion. 


\section{Summary and conclusion}

Recently the greatest attention has been paid to the elucidation of LAIC mechanism, which is really a challenging topic in the field of seismo-electromagnetics and should be pursued with the interdisciplinary approach.

The topics treated in this review, are several items associated with EQs: (i) ULF/ELF radiation, (ii) ULF magnetic field depression, (iii) anomaly in SR, (iv) EQ effects on Pc 1 pulsations, (v) VLF electromagnetic emissions, (vi) EQ effect on ELF/VLF whistlers, and (vii) lightning. In this review we try to interpret each topic as an individual phenomenon, but in another sense each topic can be considered as one aspect of the interdisciplinary mechanism of the LAIC. The first four topics are rather new with the history of about 10 years, but the presence of those electromagnetic phenomena and their possible association with EQs are found to be highly acceptable from the observational point of view because of the richness in their case and statistical studies. That is, those anomalies are found to take place a few days to a few weeks prior to an EQ, which can be recommended as promising candidates for short-term EQ prediction.Then, the topic of (vi) EQ effect on VLF whistlers was first reported over 20 years ago, and only one paper has been published recently by Taiwanese scientists. The amount of observed data on this topic is not much enough, but the result seems to suggest a strong correlation of changes in whistler propagation characteristics with EQs. Finally, the topics (v) and (vii) are closely related to each other, or we can consider that these two topics are exactly the same. It seems that lightning discharges including VLF electromagnetic emissions are likely to take place a few days before an EQ, but there remain some important questions to be answered observationally. The fundamental features of VLF electromagnetic emissions are quite uncertain (either impulsive or noise-like). An additional problem is inconsistence in the VLF location that some workers suggested the source mainly in the sea, but others indicated only land source. Nearly all kinds of ULF/ELF/VLF seismogenic waves are dealt with in this review, but we have to mention that there are additional non-conventional seismogenic ULF/ELF emissions such as SR-like line emissions before an EQ (you can find more detailed description on this topic in Sorokin et al. (2015)). Even though the presence and its possible association with EQs have been convinced for nearly all of the above seven phenomena, the generation mechanism for each phenomenon remains very puzzling even though a few possible hypotheses have been proposed. There seems to be so far no phenomenon which can be interpreted in terms of any definite hypothesis. However, all of the effects can be explained with 
one of the following two possibilities. One is the ionospheric perturbation, and the other is atmospheric discharges. Further understanding of each phenomenon will be made only with the coordinated measurement of multiple parameters just as in the case of the investigation of LAIC process of our greatest concern.

\section{Acknowledgements}

This review is based on a lot of works done with many collaborators, but we would like to dedicate this review to two of our close collaborators, the late Prof. Oleg Molchanov (deceased in 2011) and the late Prof. Kenji Ohta (deceased in 2019) for their excellent contributions to seismo electromagnetic studies.

\section{References}

Alperovich, L., and I. Chaikovsky (1995), On effective conductivity of the ionosphere with random irregularities, Ann. Geophys., 13, 339.

Alperovich, L. S., and E. N. Fedorov (2007), Hydromagnetic Waves in the Magnetosphere and theIonosphere, Series: Astrophysics and Space Science Library, vol. 353, Springer, 418p, ISBN: 978-14020-6636-8.

Asada, T., H. Baba, M. Kawazoe, and M. Sugiura (2001), An attempt to delineate very low frequency electromagnetic signals associated with earthquakes, Earth Planets Space, 53, 55-62.

Asai, S., S. Yamamoto, Y. Kasahara, Y. Hobara, T. Inaba, and M. Hayakawa (2011), Measurement of Doppler shifts of short-distance subionospheric LF transmitter signals and seismic effects, J. Geophys. Res., doi:10.1029/2010JA016055.

Biagi, P. F., R. Piccolo, L. Castellana, A. Ermini, S. Martellucci, C. Bellecci, V. Capozzi, G. Perna, O.A. Molchanov and M. Hayakawa (2004), Variations in a LF radio signal on the occasion of the recent seismic and volcanic activity in Southern Italy, Phys. Chem. Earth, 29, 551-557.

Bortnik, J., J. W. Cutler, C. Dunson, and T.E. Bleier (2008), Thepossible statistical relation of Pc 1 pulsations to earthquake occurrence at low latitudes, Ann. Geophysicae, 26, 2825-2836.

Chakrabarti, S. K. (Ed)(2010), Propagation Effects of Very Low Frequency Radio Waves, AIP (American Institute of Physics) Conference Proceeding, no. 1286, 362p.

Chmyrev, V. M., N. B. Isaev, S. V. Bilichenko, and G. Stanev (1986), Electric fields and hydromagnetic waves in ionosphere above the focus of earthquake, Geomagnetism and Aeronomy, 26, 1020-1022. 
Christofilakis, V., G. Tatsis, C. Votis, I. Contopoulos, C. Repapis, and V. Tritakis (2019), Significant ELF perturbations in the Schumann resonance band before and during a shallow mid-magnitude seismic activity in the Greek area (Kalpuki), J. Atmos. Solar-terr. Phys., 182, 138-146.

Console, R. (2001), Testing forecast earthquake hypotheses, Tectonophysics, 338, 261-268.

Davies, K., and D. M. Baker (1965), Ionospheric effects observed around the three Alaskan earthquakes of March 28, 1964, J. Geophys. Res., 70, 2251-2253.

Denisenko, V. V. (2015), Estimate for the strength of the electric field penetration from the Earth's surface to the ionosphere, Russian J. Phys. Chem., 70, 2251-2253.

Devi, M., A. K. Barbara, A. Depueva, Ya Yu, Ruzhin, and V. Depueva (2010), Anomalous total electron content (TEC) and atmospheric refractivity prior to the very strong China earthquake of May 2008, International Journal of Remote Sensing, 31, No. 13, 3589-3599.

Dreizin, Yu. A., and A. M. Dykhne (1973), Anomalous conductivity of inhomogeneous media in strong magnetic field, Soviet Phys. JETP, 36, 127-136.

Fenoglio, M. A., M. J. S. Johnston, and J. D. Byerlee (1995), Magnetic and electric fields associated with changes in high pore pressure in fault zone -application to the Loma Prieta ULF emissions, J. Geophys. Res., Solid Earth, 100, 12951-12958.

Fowler, R. A., B. J. Kotick, and R. D. Elliot (1967), Polarization analysis of natural and artificially induced geomagnetic micropulsations, J. Geophys. Res., 72, 2871-2875.

Fraser-Smith, A. C., A. Bernardi, P. R. McGill, M. E. Ladd, R. A. Helliwell, and O. G. Villard Jr. (1990), Low-frequency magnetic field measurements near the epicenter of the Ms 7.1 Loma Prieta earthquake, Geophys. Res. Lett., 17, 1465-1468.

Freund, F. (2009), Stress-activated positive hole charge carriers in rocks and the generation of preearthquake signals, in "Electromagnetic Phenomena Associated with Earthquakes", Ed. by M. Hayakawa, Transworld Research Network, Trivandrum, India, 41-96.

Fujinawa, Y., K. Takahashi, T. Matsumoto, and N. Kawakami (1999), Sources of earthquake-related VLF electromagnetic signals, in "Atmospheric and Ionospheric Electromagnetic Phenomena Associated with Earthquakes,” Ed. by M. Hayakawa, Terra Scientific Publishing Company, Tokyo, 405-415.

Fullekrug, M., E. A. Mareev, and M. J. Rycroft (Eds) (2006), Sprites, Elves, and Intense Lightning Discharges, NATO Science Series, Springer, 398p.

Gazquez, J.A., R. M. Garcia, N. N. Castellano, M. Fernandez-Ros, A. J. Perea-Moreno, and F. ManzanoAgugliaro (2017), Applied engineering using Schumann resonance for earthquake monitoring, Applied Sciences, 7, 1113, doi: 10.3390/ app7111113. 
Gokhberg, M. B., V. A. Morgounov, T. Yoshino, and I. Tomizawa (1982), Experimental measurement of electromagnetic emissions possibly related to earthquakes in Japan, J. Geophys. Res., 87 (B9), 7824-7827.

Gokhberg, M. B., V. A. Morgounov and O. A. Pokhotelov (1995), Earthquake Prediction. SeismoElectromagnetic Phenomenon, Gordon and Breach Sci. Pub., Amsterdam, 287p.

Greifinger, C. and P. Greifinger (1978), Approximate method for determining ELF eigen-values in the Earth-ionosphere waveguide, Radio Sci., 13, 831-837.

Guglielmi, A., A. Potapov, B. Tsegmed, et al.(2006), On the earthquake effect in the regime of ionospheric Alfven resonances, Phys. Chem. Earth, 31, 469-472.

Guglielmi., A. V., and O. D. Zotov (2010), Correlation between Pc 1 electromagnetic activity and earthquakes, Izvestiya, Phys. of the Earth, 46, 486-492.

Hata, M., K. Ohta, J. Izutsu, I. Takumi, T. Fujii, T. Sato, S. Yanashi, and N. Watanabe (2010), Development of ULF band receiver for detecting electromagnetic wave precursor of earthquakes, $\mathrm{J}$. Atmos. Electr., 31, 13-36.

Hattori, K. (2013), ULF geomagnetic changes associated with earthquakes, in "Earthquake Prediction Studies: Seismo Electromagnetics”, Ed. by M. Hayakawa, TERRAPUB Tokyo, 129-152.

Hayakawa, M. (1995), Whistlers, in "Handbook of Atmospheric Electrodynamics", Ed. by H.Volland, CRC Press, Boca Raton, vol. 1, 155-193.

Hayakawa, M. (Ed) (1999), "Atmospheric and Ionospheric Phenomena Associated with Earthquakes", TERRAPUB, Tokyo, 966p.

Hayakawa, M. (2011), Probing the lower ionosphere by means of subionospheric VLF propagation, Earthq. Sci., 24, no.6, 609-637.

Hayakawa, M. (2015), Earthquake Prediction with Radio Techniques, John Wiley \& Sons, Singapore, $294 p$.

Hayakawa, M., J. Ohtsu, and A. Iwai (1971), Characteristics of dispersion and occurrence rate of whistlers at low latitudes during one solar cycle, J. Geomagn. Geoelectr., 23, 187-204.

Hayakawa, M. and J. Ohtsu (1973), Ducted propagation of low-latitude whistlers deduced from simultaneous observations at multi-stations, J. Atmos. Terr. Phys., 35, 1685-1697.

Hayakawa, M. and Y. Tanaka (1978), On the propagation of low-latitude whistlers, Rev. Geophys. Space Phys., 16, 111-123. 
Hayakawa, M., K. Ohta, and S. Shimakura (1990), Spaced direction finding of very low latitude whistlers and their propagation mechanism, J. Geophys. Res., 95, 15091-15102.

Hayakawa, M. and S. S. Sazhin (1992), Mid-latitude and plasmaspheric hiss: A review, Planet. Space Sci., 40, 1325-1338.

Hayakawa, M., T. Yoshino, and V. A. Morgounov (1993), On the possible influence of seismic activity on the propagation of magnetospheric whistlers at low latitudes, Phys. Earth Planet. Inter., 77, 97-108.

Hayakawa, M. and Y. Fujinawa (Eds) (1994), Electromagnetic Phenomena Related to Earthquake Prediction, Terra Sci. Pub. Co., Tokyo, 667p.

Hayakawa, M., O. A. Molchanov, T. Ondoh, and E. Kawai (1996a), The precursory signature effect of the Kobe earthquake on VLF subionospheric signals, J. Comm. Res. Lab., Tokyo, 43, 169-180.

Hayakawa, M., R. Kawate, O. A. Molchanov, and K. Yumoto (1996b), Results of ultra-low-frequency magnetic field measurements during the Guam earthquake of 8 August 1993, Geophys. Res. Lett., 23, 241-244.

Hayakawa M. and O. Molchanov (Eds) (2002), Seismo Electromagnetics: Lithosphere-AtmosphereIonosphere Coupling, TERRAPUB, Tokyo, 477p.

Hayakawa, M., O. A. Molchanov, and A. P. Nickolaenko (2002), Model variation in atmospheric radio noise caused by pre-seismic modifications of tropospheric conductivity profile, in "Seismo Electromagnetics:Lithosphere-Atmosphere-Ionosphere Coupling", Ed. by M. Hayakawa and O. A. Molchanov, TERRAPUB, Tokyo, 349-352.

Hayakawa, M., O. A. Molchanov, NASDA/UEC team (2004), Summary report of NASDA's earthquake remote sensing frontier project, Phys. Chem. Earth, 29, 617-625.

Hayakawa, M., K. Ohta, A.P. Nickolaenko, and Y. Ando (2005), Anomalous effect in Schumann resonance phenomena observed in Japan, possibly associated with the Chi-chi earthquake in Taiwan, Ann. Geophysicae, 23, 1335-1346.

Hayakawa. M., A. P. Nickolaenko, M. Sekiguchi, K. Yamashita, Y. Ida, and M. Yano (2008), Anomalous ELF phenomena in the Schumann resonance band as observed at Moshiri (Japan) in possible association with an earthquake in Taiwan, Natural Hazards Earth System Sci., 8, 1309-1316.

Hayakawa, M., Y. Kasahara, T. Nakamura, F. Muto, T. Horie, S. Maekawa, Y. Hobara, A. A., Rozhnoi, M. Solovieva, and O. A. Molchanov (2010), A statistical study on the correlation between lower ionospheric perturbations as seen by subionospheric VLF/LF propagation and earthquakes, J. Geophys. Res., 115, A09305, doi:10.1029/2009JA015143. 
Hayakawa, M., Y. Hobara, K. Ohta, and K. Hattori (2011a), The ultra-low-frequency magnetic disturbances associated with earthquakes, Earthq. Sci., 24, 523-534.

Hayakawa, M., Y. Kasahara, T. Nakamura, Y. Hobara, A. Rozhnoi, M. Solovieva, O. A. Molchanov, and V. Korepanov (2011b), Atmospheric gravity waves as a possible candidate for seismo-ionospheric perturbations, J. Atmos. Electr., 31, No. 2, 129-140.

Hayakawa, M., A. Rozhnoi, M. Solovieva, Y. Hobara, K. Ohta, A. Schekotov, and E. Fedorov (2013a), The lower ionospheric perturbation as a precursor to the 11 March 2011 Japan earthquake, Geomatics, Natural Hazards and Risk, doi:org/10.1080/19475705.2012.751938, 1-13.

Hayakawa, M., A. Schekotov, E. Fedorov, and Y. Hobara (2013b), On the ultra-low-frequency magnetic field depression for three huge oceanic earthquakes in Japan and in the Kurile islands, Earth Science Research, 2, 33-42.

Hayakawa, M., T, Asano, A. Rozhnoi, and M. Solovieva (2018), Very-low- and low-frequency sounding of ionospheric perturbations and possible association with earthquakes, in "Pre-Earthquake Processes: A multidisciplinary approach to earthquake prediction studies", Ed. by D. Ouzounov et al., AGU, Wiley, Chapter 16, 277-304.

Helliwell, R. A. (1965), Whistlers and Related Ionospheric Phenomena, Stanford Univ. Press, 1965.

Hughes W. J., and D. J. Southwood (1976), The screening of micropulsation signals by the atmosphere and ionosphere, J. Geophys. Res., 81, 3234-3240.

Jacobs, J. A. (1970), Geomagnetic Micropulsations, Springer-Verlag.

Kopytenko, Yu. A., T. G. Matiashvily, P. M. Voronov, E. A. Kopytenko, and O. A. Molchanov (1990), Detection of ULF emission connected with the Spitak earthquake and its aftershock activity based on geomagnetic pulsations data at Dusheti and Vardziya observatories, Phys. Earth Planet. Intrer., 77, 85-95.

Korepanov, V., M. Hayakawa, Y. Yampolski, and G. Lizunov (2009), AGW as a seismo-ionospheric coupling responsible agent, Phys. Chem. Earth, 34, Issues 6-7, 485-495.

Liu, J. Y. (2009), Earthquake precursors observed in the ionospheric F-region, in "Electromagnetic Phenomena Associated with Earthquakes", Ed. by M. Hayakawa, Transworld Research Network, Trivandrum, India, 187-204.

Liu, J. Y., K. Wang, C. H. Chen, W. H. Yang, Y. H. Yen, Y. I. Chen, K. Hattori, H. T. Su, R. R. Hsu, and C. H. Chang (2013), A statistical study on ELF-whistlers/emissions and $\mathrm{M} \geq 5.0$ earthquakes in Taiwan, J. Geophys. Res., 118, 3760-3768, doi : 10.1002/jgra. 50356.

Liu, J. Y., K. Hattori, and Y. I. Chen (2018), Application of total electron content derived from the global navigation satellite system for detecting earthquake precursors, in "Pre-Earthquake Processes; A 
multidisciplinary approach to earthquake prediction studies, Ed. by D. Ouzounov et al., AGU Monograph, 305-317.

Liu, J. Y., Y. I. Chen, Y. J. Chou, and H. F. Tsai (2001), Variations of ionospheric total electron content during the Chi-chi earthquake, Geophys. Res. Lett., 28, 1383-1386.

Liu, J. Y., Y. I. Chen, C. H. Huang, Y. Y. Ho, and C. H. Chen (2015), A statistical study of lightning activities and $M>5.0$ earthquakes in Taiwan during 1993-2004, Surveys in Geophys., 36, 851-859, doi: 10.1007/s10712-015-9342-2.

McCormick, R. J., C. J. Rodger, and N. R. Thomson (2002), Reconsidering the effectiveness of quasistatic thunderstorm electric fields for whistler duct formation, J. Geophys. Res., Space Physics,107, doi.org/10.1029/2001JA009219.

Manchester, R. N. (1966), Propagation of Pc 1 micropulsations from high to low latitudes, J. Geophys. Res., 71, 3749-3754.

Mareev, E. A., D. I. Iudin, and O. A. Molchanov (2002), Mosaic source of internal gravity waves associated with seismic activity, in "Seismo Electromagnetics: Lithosphere-Atmosphere-Ionosphere Coupling”, Ed. by M. Hayakawa, M. and O. A. Molchanov, TERRAPUB, Tokyo, 335-342.

Matsumoto, T., Y. Fujinawa, and K. Takahashi (1996), ULF-bands electric field changes related to the seismic swarm, J. Atmos. Electr., 16, 175-191.

Merzer, I., and S. L. Klemperer (1997), Modeling low-frequency magnetic field precursors to the Loma Prieta earthquake with a precursory increase in fault-zone conductivity, Pure Appl. Geophys., 150, $217-$ 248.

Miyaki, K., M. Hayakawa, and O. A. Molchanov (2002), The role of gravity waves in the lithosphere ionosphere coupling, as revealed from the subionospheric LF propagation data, in "Seismo Electromagnetics: Lithosphere - Atmosphere - Ionosphere Coupling”, Ed. by M. Hayakawa and O. A. Molchanov, TERRAPUB, Tokyo, 229-232.

Molchanov, O. A., Yu. A. Kopytenko, P. M. Voronov, E. A. Kopytenko, T. G. Matiashvily, A. C. FraserSmith, and A. Bernardi (1992), Results of ULF magnetic field measurements near the epicenters of the Spitac (Ms=6.9) and Loma Prieta (Ms=7.1) earthquakes: comparative analysis, Geophys. Res. Lett., 19, 1495-1498.

Molchanov, O. A. and M. Hayakawa (1995), Generation of ULF electromagnetic emissions by microfracturing, Geophys. Res. Lett., 22, 3091-3094.

Molchanov, O. A. and M. Hayakawa (1998), Subionospheric VLF signal perturbations possibly related to earthquakes, J. Geophys. Res., 103, 17,489-17,504. 
Molchanov, O. A., M. Hayakawa, and K. Miyaki (2001), VLF/LF sounding of the lower ionosphere to study the role of atmospheric oscillations in the lithosphere-ionosphere coupling, Adv. Polar Upper Atmos. Res., 15, 146-158.

Molchanov, O. A., O. A. Mazhaeva, A. N. Goliavin, and M. Hayakawa (1993), Observations by the intercosmos-24 satellite of ELF-VLF electromagnetic emissions associated with earthquakes, Ann. Geophysicae, 11, 431-440.

Molchanov, O. A., A. Yu. Schekotov, E. N. Fedorov, G. G. Belyaev, and E. E. Gordeev (2003), Preseismic ULF electromagnetic effect from observation at Kamchatka, Natural Hazards Earth System Sci., 3, 1 - 7 .

Molchanov, O. A., A. Yu. Schekotov, E. Fedorov, G. N. Belyaev, M. S. Solovieva and M. Hayakawa (2004), Preseismic ULF effect and possible interpretation, Ann. Geophys., 47, 119-131.

Molchanov, O. A., and M. Hayakawa (2008), Seismo-Electromagnetics and Related Phenomena: History and latest results, TERRAPUB, Tokyo, $189 \mathrm{p}$.

Morgounov, V. A. (1985), Electromagnetic emissions during seismic activity, Izvestiya Academy of Sciences of USSR, Physics of Solid Earth, 21, 220-226 (in English).

Mushtak, V. C. and E. R. Williams (2002), ELF propagation parameters for uniform models of the Earthionosphere waveguide, J. Atmos. Solar-terr. Phys., 64, 1989-2001.

Nakamura, T., V. Korepanov, Y. Kasahara, Y. Hobara, and M. Hayakawa (2013), An evidence on the lithosphere-ionosphere coupling in terms of atmospheric gravity waves on the basis of a combined analysis of surface pressure, ionospheric perturbations and ground-based ULF variations, J. Atmos. Electr., 33, 53-68.

Nickolaenko, A.P. and M. Hayakawa (2002), Resonances in the Earth-Ionosphere Cavity, Kluwer Acad. Pub., Dordrecht, 380p.

Nickolaenko, A. P., and M. Hayakawa (2014), Schumann Resonances for Tyros: Essentials of Global Electromagnetic Resonance in the Earth-Ionosphere Cavity, Springer, Tokyo, 348p.

Nickolaenko, A. P., M. Hayakawa, M. Sekiguchi, Y. Ando, and K. Ohta (2006), Model modifications in Schumann resonance intensity caused by a localized ionosphere disturbance over the earthquake epicenter, Ann. Geophysicae, 24, 567-575.

Nishida, A.(1972), Geomagnetic Diagnosis of the Magnetosphere, Springer, New York.

Ogawa, T., Y. Tanaka, A. C. Fraser-Smith, and R. Gendrin (1967), Worldwide simultaneity of a Q-burst in the Schumann resonance frequency range, J. Geomagn. Geolectr., 19, 377-384. 
Ohta, K., and M. Hayakawa (1990), The correlation of whistler occurrence rate at a low latitude with thunderstorm activity at its conjugate region and with solar activity, Pure Appl.Geophys., 133, 167-178.

Ohta, K., M. Hayakawa, and Y. Tanaka (1984), Ducted propagation of daytime whistlers as deduced from the ground-based direction finding, J. Geophys. Res., 89, 7557-7564.

Ohta, K., N. Watanabe and M. Hayakawa (2006), Survey of anomalous Schumann resonance phenomena observed in Japan, in possible association with earthquakes in Taiwan, Phys. Chem. Earth, 31, 397-402.

Ohta, K., J. Izutsu, and M. Hayakawa (2009), Anomalous excitation of Schumann resonances and additional anomalous resonances before the 2004 Mid-Niigata prefecture earthquake and the 2007 Noto Hantou earthquake, Phys. Chem. Earth, 34, 441-448.

Ohta, K., J. Izutsu, A. Schekotov, and M. Hayakawa (2013), The ULF/ELF electromagnetic radiation before the 11 March 2011 Japanese earthquake, Radio Sci., 48, 589-596, doi:10.1002/ rds.20064.

Oike, K., and T. Yamada (1994), Relationship between shallow earthquakes and electromagnetic noises in the LF and VLF ranges, in "Electromagnetic Phenomena Related to Earthquake Prediction," Ed. by M. Hayakawa, and Y. Fujinawa, Terra Scientific Publishing Company, Tokyo, 115-130.

Okada, T., A. Iwai, and M. Hayakawa (1977), The measurement of incident and azimuthal angles and the polarization of whistlers at low latitudes, Planet. Space Sci., 25, 233-241.

Ouyang, X., X. Zhang, Nickolaenko, A. P., Hayakawa, M., X. Shen, and Y. Miao (2013). Schumann resonance observation in China and anomalous disturbance possibly associated with Tohoku M 9.0 earthquake, Earthq. Sci., 26 (2), 137-145.

Ouzounov, D., S. Pulinets, K. Hattori, and P. Taylor (Eds) (2018), Pre-Earthquake Processes: A multidisciplinary approach to earthquake prediction studies, AGU Geophysical Monograph 234, Wiley, $365 \mathrm{p}$.

Park, C. G. (1982), Whistlers, in "Handbook on Atmospherics," Ed. by H. Volland, vol. 2, CRC Press, Boca Raton, 21-77.

Park, C. G., and R. A. Helliwell (1971), The formation of field-aligned irregularities in the magnetosphere, Radio Sci., 6(2), 299-340.

Park, S. K. (1997), Electromagnetic precursors to earthquakes: a search for predictors, Science Progress, $80,65-82$.

Parrot, M., and M. Li (2018), Statistical analysis of the ionospheric density recorded by the DEMETER satellite during seismic activity, in "Pre-Earthquake Processes: A multi disciplinary approach to earthquake prediction studies", Ed. by D. Ouzounov et al., AGU Monograph, 319-328. 
Pilipenko, V. A. (2012), Impulsive coupling between the atmosphere and ionosphere/magnetosphere, Space Sci. Rev., 168, 533-550.

Price, C. (2016), ELF electromagnetic waves: Schuman resonances, Atmosphere, 7(9), 116; doi:10.3390/atmos7090116.

Prokhorov, B. E., and O. V. Zolotov (2017), Comment on "An improved coupling model for the lithosphere-atmosphere-ionosphere coupling system” by Kuo et al. (2014), J. Geophys. Res., Space Phys., 122, 4865- 4868, doi: 10.1002/2016 JA 023441.

Pulinets, S., and K. Boyarchuk (2004), Ionospheric Precursors of Earthquakes, Springer, Berlin, 315p.

Pulinets, S. and Ouzounov, D. (2011), Lithosphere-atmosphere-ionosphere coupling (LAIC) model- a unified concept for earthquake precursors validation, J. Asian Earth Sci., 41, 371-382.

Rozhnoi, A., M. S. Solovieva, O. A. Molchanov and M. Hayakawa (2004), Middle latitude LF (40 kHz) phase variations associated with earthquakes for quiet and disturbed geomagnetic conditions, Phys. Chem. Earth, 29, 589-598.

Rozhnoi, A., M. Solovieva, and M. Hayakawa (2013), VLF/LF signals method for searching of electromagnetic earthquake precursors, in "Earthquake Prediction Studies: Seismo Electromagnetics", Ed. by M. Hayakawa, TERRAPUB, Tokyo, 31-48.

Ruzhin, Yu.Ya., and A. Kh. Depueva (1996), Seismo-precursors in space as plasma and wave anomalies, J. Atmos. Electr., 16, 271-288.

Rycroft, M. J., K. A. Nicoll, K. L. Aplin, and R.G. Harrison (2012), Recent advances in global electric circuit coupling between the space environment and the troposphere, J. Atmos. Solar-terr. Phys., 9091,198-211.

Saito, T. (1969), Geomagnetic pulsations, Space Sci. Rev., 10, 319-412.

Sazhin, S. S., and M. Hayakawa (1992), Magnetospheric chorus emissions: A review, Planet. Space Sci., 40, 681-697.

Sazhin, S. S., K. Bullough, and M. Hayakawa (1993), Auroral hiss: A review, Planet. Space Sci., 41, 153 166.

Schekotov, A., O. Molchanov, K. Hattori, E. Fedorov, V. A. Gladyshev, G. G. Belyaev, V. Chebrov, V. Sinitsin, E. Gordeev, and M. Hayakawa (2006), Seismo-ionospheric depression of the ULF geomagnetic fluctuations at Kamchatka and Japan, Phys. Chem. Earth, 31, 313-318. 
Schekotov, A. Y., O. A. Molchanov, M. Hayakawa, E. N. Fedorov, V. N. Chebrov, V. I. Sinitsin, E. E. Gordeev, G. G. Belyaev, and N. V. Yagova (2007), ULF/ELF magnetic field variations from atmosphere induced by seismicity, Radio Sci., 42, RS6S90, doi:10.1029/2005RS003441.

Schekotov, A. Y., O. A. Molchanov, M. Hayakawa, E. N. Fedorov, V. N. Chebrov, V. I. Sinitsin, E. E. Gordeev, S. E. Andreevsky, G. G. Belyaev, N. V. Yagova, V. A. Gladishev, and L. N. Baransky (2008), About possibility to locate an EQ epicenter using parameters of ELF/ULF preseismic emission, Natural Hazards Earth System Sci., 8, 1237-1242.

Schekotov, A., and M. Hayakawa (2017), ULF/ELF Electromagnetic Phenomena for Short-term Earthquake Prediction, LAP Lambert Academic Publishing, Beau Bassin, Mauritius, 102p.

Schekotov, A., E. Fedorov, O. A. Molchanov, and M. Hayakawa (2013), Low frequency electromagnetic precursors as a prospect for earthquake prediction, in "Earthquake Prediction Studies: Seismo Electromagnetics”, Ed. by M. Hayakawa, TERRAPUB, Tokyo, 81-99.

Sentman, D. (1995), Schumann resonances, in "Handbook of Atmospheric Electrodynamics", Ed. by H. Volland, vol.1, 267-310, CRC Press, Boca Raton.

Simoes, F., R. Pfaff, Berthelier, J-J., and J. Klenzing (2012), A review of low frequency electromagnetic wave phenomena related to tropospheric- ionospheric coupling mechanisms, Space Sci. Rev., 168, 551593.

Singh, B., M. Hayakawa, P. K. Mishra, R. P. Singh and D. R. Lakshmi (2003), VLF electromagnetic noise bursts observed in a borehole and their relation with low-latitude hiss, J. Atmos. Solar-terr. Phys., 65, 269-276.

Sorokin, V. M., A. K. Yaschenko, and M. Hayakawa (2006), Formation mechanism of the lowerionospheric disturbances by the atmosphere electric current over a seismic region, J. Atmos. Solar-terr. Phys., 68, 1260-1268.

Sorokin, V. and M. Hayakawa (2013), Generation of seismic-related DC electric fields and lithosphereatmosphere-ionosphere coupling, Modern Appl. Sci., 7, no. 6, 1-25, doi: 10.5539/mas.v7n6p1.

Sorokin, V., V. Chemyrev, and M. Hayakawa (2015), Electrodynamic Coupling of LithosphereAtmosphere-Ionosphere of the Earth, Nova Science Pub. Inc., New York, 326p.

Surkov, V., and M. Hayakawa (2014), Ultra and Extremely Low Frequency Electromagnetic Fields, Springer, Tokyo, 486p.

Tsuruda, K., and K. Hayashi (1975), Direction finding technique for elliptically polarized VLF electromagnetic waves and its application to the low-latitude whistlers, J. Atmos. Terr. Phys., 39, 11931202. 
Tsutsui, M. (2012), For detecting electromagnetic pulses excited by impact to earth crusts, in "TheFrontier of Earthquake Prediction Studies, “Ed. by M. Hayakawa, Nihon-Senmontosho-Shuppan, Tokyo, 364-387 (in Japanese).

Weaver, P. F., P. C. Yuen, W. Proless, and A. S. Furumoto (1970), Acoustic coupling into the ionosphere from seismic waves of the earth at Kurile Islands on August 11, 1969, Nature, 226, 1239-1241.

Williams, E. R. (1992), The Schumann resonances: a global tropical thermometer, Science, 256, 11841188.

Yang, S. S., T. Asano, and M. Hayakawa (2019), Abnormal gravity wave activity in the stratosphere prior to the 2016 Kumamoto earthquakes, J. Geophys. Res. Space Phys., 124, https:// doi.org/10.1029/2018JA026002.

Yasuoka, Y. (2012), Radon anomalies prior to earthquakes, in "The Frontier of Earthquake Prediction Studies,” Ed. by M. Hayakawa, Nihon-Senmontosho-Shuppan, Tokyo, 410-427 (in Japanese).

Yoshino, T., I. Tomizawa, and T. Shibata (1985), The possibility of using a direction finding technique to locate earthquake epicenters from electromagnetic precursor radiation, Ann. Geophys., 3, 727-730. 